DRIFT BOTTLE RECORDS

FOR GULF OF MAINE

AND GEORGES BANK, 1931-56

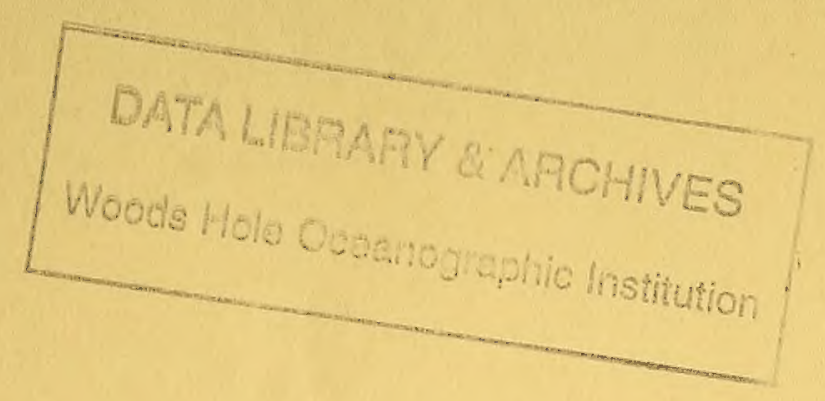

SPECIAL SCIENTIFIC REPORT-FISHERIES NNo. 242

GC

272

B8

1957
UNITED STATES DEPARTMENT OF THE INTERIOR FISH AND WILDLIFE SERVICE 


\section{EXPLANATORY NOTE}

The series embodies results of investigations, usually of restricted scope, intended to aid or direct management or utilization practices and as guides for administrative or legislative action. It is issued in limited quantities for official use of Federal, State or cooperating agencies and in processed form for economy and to avoid delay in publication. 
United States Department of the Interior, Fred A. Seaton, Secretary Fish and Wildife Service, Arnie J. Suomela, Commissioner

DRIFT BOTTLE RECORDS FOR GULF OF MAINE AND GEORGES BANK, 1931-56

by

Dean F. Bumpus, Oceanographer, and

C. Godfrey Day, Research Assistant

Woods Hole Oceanographic Institution

Special Scientific Report--Fisheries No. 242

Contribution No. 941 from the Woods Hole Oceanographic Institution

Washington, D. C.

November 1957 
TABLE OF CONTENT

Page

The data.

Bottle type and distribution ................ 4

Literature cited . . . . . . . . . . . . . 6 


\section{Drift Bottle Records for Gulf of Maine and Grorges Bank, 1931-56}

The drift-bottle data tabulated bere are the result of extended studies by the United States Bureau of Fisheries and more recently by the United States Fish and Hildife Service in the region of Georges Bank and the Gulf of Maine (fig. 1).

The apparent decline of the cod and haddock in this area has demanded attention over a period of years. Fishery biologists and oceanographers have been making concerted efforts to understand better the many complex, interrelated factor which influence the productivity of these fisheries.

One aspect of these studies has been an attempt by means of drift bottles to learn the nontidal movement of the waters bearing the planktonic eggs and larvae of comercially important fish which spawn in the region.

By the end of June 1956, 17 drift-bottle seedings had been made, representing the years 1931, 1932, 1933, 1934, 1953, 1955, and 1956. Welford (1938) discussed the bottle returns from the 1931 and 1932 cruises. Day (in press) made comparisons on a seasonal and year-to-year basis of all 17 cruises and considered surface-wind values for a period of 60 days pollowing each bottle seeding. 


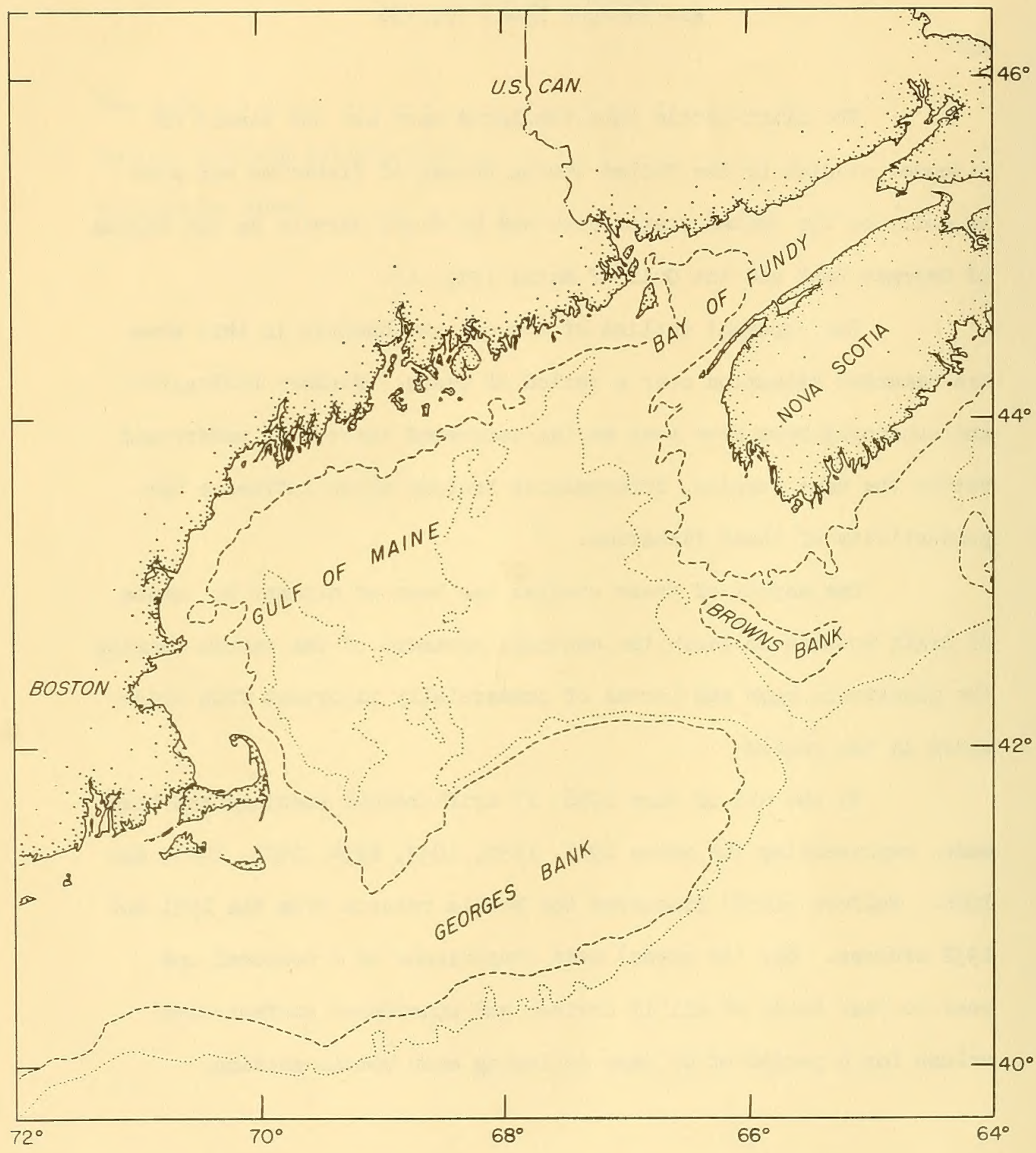


The original data are on Ille at the Woods Hole Oceanographic Institution, where a punchcard system is malntained and supplemented as new returns are recelved or further studies initiated.

The listings below include all recoveries on record as of 5 December 1956 in the Georges Bank - Gulf of Maine area Irom the following crulses:

1931 Albatross II, 17-29 April

Albatross II, 26 May - 7 June

1932. Albatross II, 4-18 April

1933 Atlantis, cruise 16, 19-28 June

1934 Atlantis, cruise 28, 27 April - 13 May

1953 Albatross III, cruise 46, 19 March - 2 April

Albatross III, cruise 48, 24 April - 8 May

Albatross III, cruise 50, 25 May - 2 June

1955 Albatross III, cruise 57, 2l February - 2 March

Albatross III, cruise 58, 19 March - 1 April

Albatross III, cruise 60, 19 April - 3 May

Albatross III, cruise $61,16-28$ May

1956 Albatross III, cruise 71, 20 February - 2 March

Albatross III, cruise 72, 2l-31 March

Albatross III, cruise 73, 17-28 April

Albatross III, cruise $75,16-29$ Mary

Albatross III, cruise $76,11-24$ June 
Returns received between 5 December 1956 and 21 October 1957 are listed in the Appendix.

The records for the cruises made during the 1930 's are believed complete so far as returns are concerned. However, the originel logs do not adequately record all release positions; consequently, In some instances positions of bottle-drop stations from which there were no returns cannot be listed. This is particularly true in the cases where bottles were distributed in pairs every 2 miles along a track.

Data for the Albatross III cruises made during the 1950 's are complete in all respects both as to releases and recoveries. During this period bottles were released only at stations, which formed a fairly unfform grid over the area of study.

\section{BOTTLE TYPE ARD DISTRIBUTION}

The bottles used were those cammonly employed for carbonated drinks. They were of clear glass and measured approximately $22 \mathrm{~cm}$. high and $6 \mathrm{~cm}$. In diameter with a capacity of 8 iluid ounces. of the bottles released on the $1931-34$ cruises, most were equipped with drogues in an effort to reduce windage. More often than not, however, the drogue had been lost sametime before the botile was recovered.

In Iigure 2 the components of the bottles used during the 1950 's are shown; a completed drift bottle is shown at the right. 


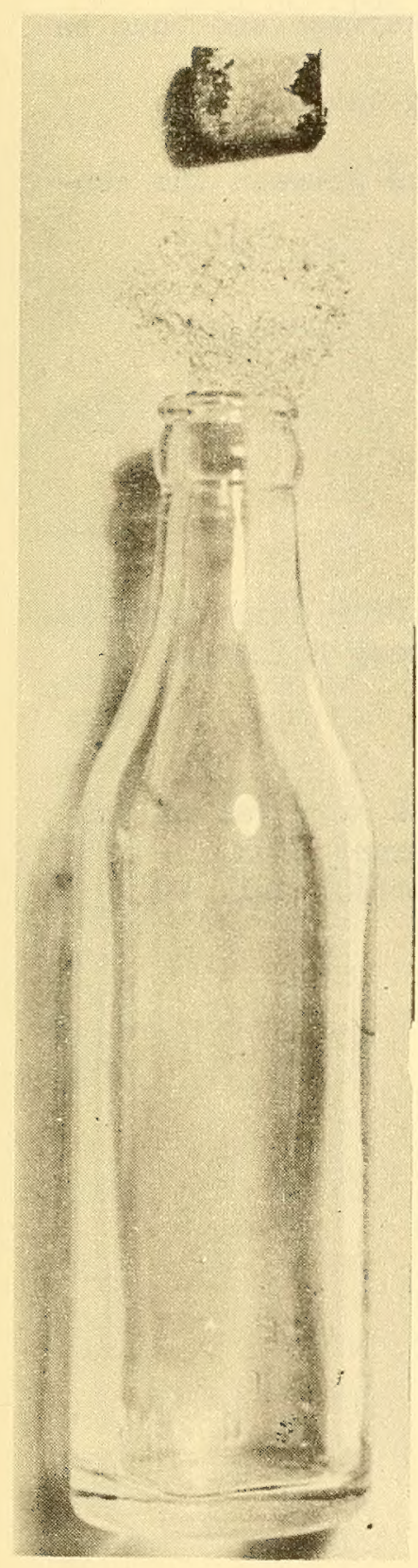

\section{BREAK THIS BOTTLE}

This bollte is une of several hundred relewed at we-s to study arean currents. The exart placie and dilte uf releake has been recorded and is on bile.

You can make the record for this hotlle complete by tornishing the information reyursted on the ent-losed postal eard. We will send a reward of Hitly eents (s.50) and tell you where the botlle wiss dropped overboard if you will return the eard with the best information you ean grovide.

Your Information will be combined with that sent in by other finders of botlles. This is part of a study of the drift of thosting objects being conducted by the Woods Hole Oeeanosraphic Institution, the Fish and wudits Service and the Fisheries Research Board of Canada.

Your cooperation in glving accurate information will be of great ald.

FINDER OF TUIS BOTTLE PLEASE, FILL IN THE INFORMATION

AS INDICATED AND BEND BY MAL

When Pound, date

Where fuund (name' of beach or place on shore, near what

Canst Guard station. Laghthouse, or uther preminent reference point)

Your Name (sign and print)

Yeur Itume Address (print)

Tiny Cents 10.50\} will be sent to Ander on return of this card.

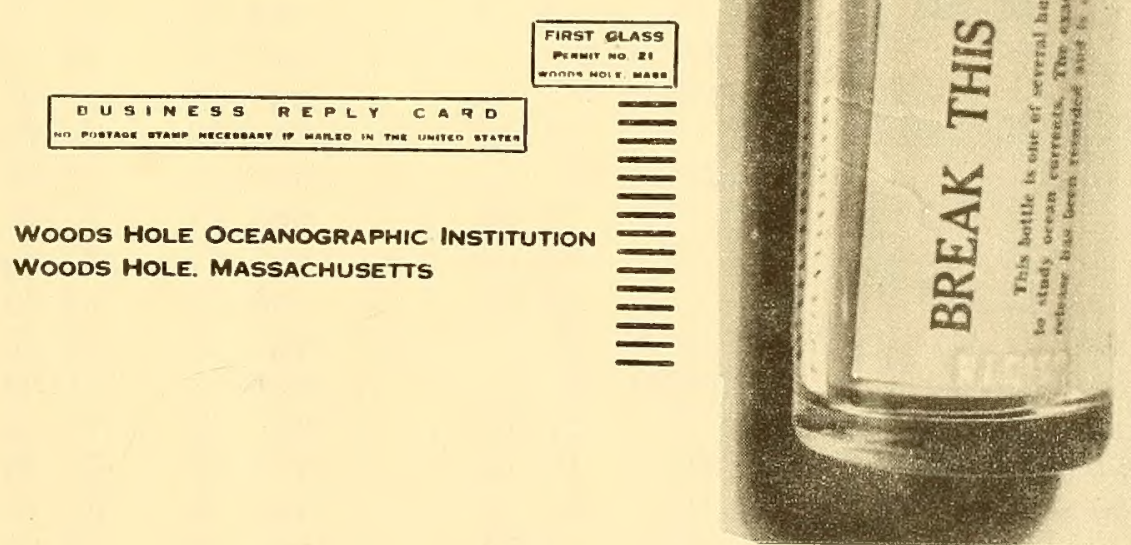


Instead of drogues, dry sand ballast was added to all odd-numbered bottles. Those with even numbers contained no ballast. Six of each were launched at each release station. It was intended that the ballasted bottles float vertically at or near the surface and thus be less influenced by the action of winds and breaking waves. The results, however, indicate no signiflcant difference between the conjectured movement of the two types.

\section{LITERATURE CITED}

Day, D. Godfrey

In press. Gulf of Maine circulation as deduced from drift bottles. U. S. Fish and Wildife Service, Fishery Bulletin. In press.

Walford, Lionel A.

1938. Effect of currents on distribution and survival of the eggs and larvae of the baddock on Georges Bank.

Bulletin of the U. S. Bureau of F1sheries, vol. 49. 
Drift Bottle Records for the Gulf of Mai ne and Georges Bank

from 1931 through 1956

Returns from 5 December 1956 to 21 October 1957

listed in Appendix

ALBATROSS II 17-29 April, 1931

10 bottles with drags released at each station

Released

Recovered

\begin{tabular}{|c|c|c|c|c|c|c|}
\hline Bottle & Lat & Long & Date & Lat & Long & Date \\
\hline & 4206 & 6954 & 17 Apr & \multicolumn{3}{|c|}{ No returns } \\
\hline 3012 & 4141 & 6939 & $17 \mathrm{Apr}$ & 4121 & 7040 & 22 JuI 31 \\
\hline 3018 & 4747 & 6939 & $17 \mathrm{Apr}$ & & 7039 & $25 \mathrm{Jul} 31$ \\
\hline 3019 & 4741 & 6939 & 17 Apr & 4130 & 7112 & 23 JUI 31 \\
\hline 3030 & 4114 & 6922 & 17 Apr & 4310 & 6538 & 28 JuI 31 \\
\hline 3037 & 4049 & 6906 & $18 \mathrm{Apr}$ & 4715 & 7005 & 16 JuI 31 \\
\hline 3038 & 4049 & 6906 & $18 \mathrm{Apr}$ & 4120 & 7049 & 31 Jul 31 \\
\hline 3040 & 4049 & 6906 & $18 \mathrm{Apr}$ & 4121 & 7039 & 26 JuI 31 \\
\hline 3047 & 4024 & 6940 & $18 \mathrm{Apr}$ & 4121 & 7051 & 1 Jun 31 \\
\hline 3060 & 4053 & 6959 & $18 \mathrm{Apr}$ & 4119 & 7049 & 25 May 31 \\
\hline 3065 & 4105 & $70 \lcm{48}$ & 20 Apr & 4127 & 7126 & 23 May 31 \\
\hline 3066 & 4105 & 70.48 & $20 \mathrm{Apr}$ & 4121 & 7030 & 1 May 31 \\
\hline 3067 & 4105 & 7048 & $20 \mathrm{Apr}$ & 4120 & 7045 & 8 May 31 \\
\hline 3068 & 4105 & 7048 & $20 \mathrm{Apr}$ & 4121 & 7027 & 12 May 31 \\
\hline 3069 & 4105 & 7048 & $20 \mathrm{Apr}$ & 4121 & 7042 & 9 May 31 \\
\hline \multirow[t]{3}{*}{3070} & 4105 & 7048 & $20 \mathrm{Apr}$ & 4721 & 7030 & 7 May 31 \\
\hline & 4043 & 7041 & $20 \mathrm{Apr}$ & \multicolumn{3}{|c|}{ No returns } \\
\hline & 4022 & 7034 & $20 \mathrm{Apr}$ & \multicolumn{3}{|c|}{ No returns } \\
\hline \multirow[t]{2}{*}{3093} & 4001 & 7029 & $20 \mathrm{Apr}$ & 4117 & $70 \quad 13$ & $5 \mathrm{Aug} 31$ \\
\hline & 3957 & 6924 & $21 \mathrm{Apr}$ & \multicolumn{3}{|c|}{ No retums } \\
\hline 3113 & 4018 & 6852 & $21 \mathrm{Apr}$ & 4037 & 7316 & $23 \mathrm{JuI} 31$ \\
\hline 3115 & 4018 & 6852 & $21 \mathrm{Apr}$ & 4045 & 7249 & 6 Sep 31 \\
\hline \multirow[t]{3}{*}{3124} & 4008 & 6849 & $21 \mathrm{Apr}$ & 7040 & $2340 \mathrm{E}$ & 5 Mar 33 \\
\hline & 4019 & 6801 & $21 \mathrm{Apr}$ & \multirow{2}{*}{\multicolumn{3}{|c|}{$\begin{array}{l}\text { No retums } \\
\text { No returns }\end{array}$}} \\
\hline & 4035 & 6811 & $22 \mathrm{Apr}$ & & & \\
\hline 3151 & 4101 & 6827 & $22 \mathrm{Apr}$ & 4135 & 7039 & 30 JuI 31 \\
\hline 3164 & 4116 & 6755 & 22 Apr & 421 & 7030 & 5 Aug 31 \\
\hline 3168 & 4116 & 6755 & $22 \mathrm{Apr}$ & 4121 & 7030 & $6 \mathrm{Aug} 31$ \\
\hline 3169 & 4116 & 6755 & $22 \mathrm{Apr}$ & 4054 & 7220 & $21 \mathrm{Jul} 31$ \\
\hline 3179 & 4049 & 6739 & $22 \mathrm{Apr}$ & 4035 & 7350 & $10 \mathrm{Jul} 31$ \\
\hline 3185 & & & 22 Apr & & 0125 & $25 \mathrm{~N}$ \\
\hline
\end{tabular}




\begin{tabular}{|c|c|c|c|c|c|c|}
\hline Bottle & Lat & Lon $\varepsilon_{2}$ & Date & Lat & Long & Date \\
\hline & 4023 & 6718 & 23 Apr & \multicolumn{3}{|c|}{ No returns } \\
\hline 3207 & $4 \mathrm{I} 04$ & $67 \mathrm{Ol}_{1}$ & $23 \mathrm{Apr}$ & 4132 & 7112 & 21 JuI 31 \\
\hline 3209 & 4104 & 6704 & 23 Apr & 4510 & 6446 & 20 Sep 31 \\
\hline 3210 & 4104 & 6704 & 23 Apr & 4210 & 6600 & 25 JuI 31 \\
\hline 3211 & 4129 & 6717 & $23 \mathrm{Apr}$ & 4119 & $70 \quad 49$ & $28 \mathrm{JuI} 31$ \\
\hline 3221 & 4144 & $6 6 \longdiv { 4 6 }$ & 23 Apr & 4033 & 6705 & 6 Jun 31 \\
\hline 3233 & 4117 & 6626 & $24 \mathrm{Apr}$ & 5900 & 0520 & 14 Sep 32 \\
\hline 3238 & 4117 & 6626 & $24 \mathrm{Apr}$ & $57 \mathrm{OL}_{4}$ & 0634 & 7 Nov 32 \\
\hline & 4705 & 6617 & 24 Apr & \multicolumn{3}{|c|}{ No returns } \\
\hline 3256 & $\begin{array}{l}4133 \\
58\end{array}$ & $\begin{array}{ll}65 & 52 \\
66 & 18\end{array}$ & $25 \mathrm{Apr}$ & 5428 & 0828 & $30 \operatorname{Oct} 32$ \\
\hline 3279 & 4200.8 & 6612 & $25 \mathrm{Apr}$ & 5240 & 0940 & 28 Sep 32 \\
\hline 3289 & $42 \mathrm{OL}^{\circ}$ & 6604.5 & $25 \mathrm{Apr}$ & 4507 & 6635 & 29 Jul 3. \\
\hline 3294 & 4206 & 6601.5 & 25 Apr & 4348 & 6632 & 24 Jun 3 \\
\hline 3295 & 4206 & 6600 & 25 Apr & 4520 & $64 \lcm{47}$ & 10 Aug 3 . \\
\hline 3307 & 4210.5 & 6551 & 25 Apr & 4330 & 6525 & 17 May 3 \\
\hline 3312 & 4212 & $6 5 \longdiv { 4 8 }$ & 25 Apr & 4500 & 6509 & 29 Sep 3] \\
\hline 3316 & 4213 & 6549 & 25 Apr & 4508 & 6453 & 19 sep 3 \\
\hline 3326 & 4216 & 6552 & $25 \mathrm{Apr}$ & 4124 & $7 0 \longdiv { 4 4 }$ & 6 Aug 3 \\
\hline 3366 & 4228.5 & 6604.5 & $25 \mathrm{Apr}$ & $44=7$ & 6642 & 3 Aug 3 \\
\hline & 4224 & 6633 & 26 Apr & \multicolumn{3}{|c|}{ No returns } \\
\hline 3386 & 4211 & 6707 & 27 Apr & 4116 & 7011 & 7 Sep 31 \\
\hline 3390 & 4211 & 6707 & 27 Apr & 4132 & 6954 & $16 \mathrm{Jul} 31$ \\
\hline 3394 & 4236 & 6717 & 28 Apr & 4130 & 7108 & 27 Sep 3I \\
\hline 3406 & 4223 & 6800 & 28 Apr & 4339 & 6506 & 31 JuI 37 \\
\hline 3417 & 4157 & 6740 & $28 \mathrm{Apr}$ & 4121 & 7039 & 3 Aug 31 \\
\hline 3430 & 4745 & 6812 & 28 Apr & 41. 18 & 7016 & $25 \operatorname{Jan} 33$ \\
\hline & 41. 29 & 6846 & 29 Apr & \multicolumn{3}{|c|}{ No returns } \\
\hline 3447 & 4755 & 6910 & 29 Apr & 4449 & 6532 & 1 Oct 31 \\
\hline
\end{tabular}

* Botties seeded in string, 2 every 2 miles. Recoveries only listed. 
10 bottles with drags released at each station.

Released

$\begin{array}{lllll}\text { Bottle } & \text { Lat } & \text { Long } & \text { Date } \\ 3459 & 4105 & 7048 & 26 \mathrm{May} \\ 3471 & 4049 & 7002 & 26 \mathrm{May} \\ 3476 & 4049 & 7002 & 26 \mathrm{May} \\ 3477 & 4049 & 7002 & 26 \mathrm{May} \\ 3478 & 4049 & 7002 & 26 \mathrm{May} \\ 3479 & 4049 & 7002 & 26 \mathrm{May} \\ 3489 & 4026 & 6939 & 26 \mathrm{May} \\ 3491 & 3957 & 6923 & 27 \mathrm{May} \\ 3508 & 4008 & 6847 & 27 \mathrm{May} \\ & 4019 & 6851 & 27 \mathrm{May} \\ 3531 & 4045 & 6908 & 27 \mathrm{May} \\ 3539 & 4115 & 6923 & 27 \mathrm{May} \\ 3540 & 4115 & 6923 & 27 \mathrm{May} \\ 3542 & 4145 & 6923 & 27 \mathrm{May} \\ 3545 & 4142 & 6940 & 28 \mathrm{May} \\ 3545 & 4112 & 6940 & 28 \mathrm{May} \\ 3548 & 4142 & 6940 & 28 \mathrm{May} \\ 3560 & 4206 & 6954 & 28 \mathrm{May} \\ 3561 & 4158.5 & 7021 & 28 \mathrm{May} \\ 3562 & 4158.5 & 7021 & 28 \mathrm{May} \\ 3563 & 4158.5 & 7021 & 28 \mathrm{May} \\ 3567 & 4158.5 & 7021 & 28 \mathrm{May} \\ 3570 & 4158.5 & 7021 & 28 \mathrm{May} \\ 3571 & 4216 & 7021 & 28 \mathrm{May} \\ 3572 & 4216 & 7021 & 28 \mathrm{May} \\ 3573 & 4216 & 7021 & 28 \mathrm{May} \\ 3575 & 4216 & 7021 & 28 \mathrm{May} \\ 3576 & 4216 & 7021 & 28 \mathrm{May} \\ 3579 & 4216 & 7021 & 28 \mathrm{May} \\ 3580 & 4216 & 7021 & 28 \mathrm{May} \\ & 4155 & 6909 & 3 \mathrm{Jun} \\ 3602 & 4130 & 6847 & 3 \mathrm{Jun} \\ 3603 & 4103 & 6831 & 3 \mathrm{Jun} \\ 3614 & 4035 & 6809 & 3 \mathrm{Jun} \\ & 4020 & 6803 & 3 \mathrm{Jun} \\ & 4031 & 6724 & 4 \mathrm{Jun}\end{array}$

Recovered

Iat Iong Date

$\begin{array}{lllllll}40 & 04 & 74 & 03 & 10 & \mathrm{Ju} & 31\end{array}$

$\begin{array}{llllll}40 & 35 & 7340 & 9 & \mathrm{Jul} & 31\end{array}$

\begin{tabular}{lllll}
40 & 40 & 73 & 04 & 28 \\
\hline & Jun 31
\end{tabular}

$4120 \quad 7142 \quad 11$ JuI 31

$4119 \quad 7049 \quad 27$ Aug 31

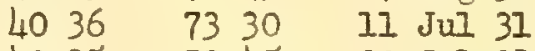

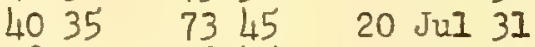

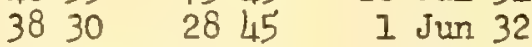

$4116.57013 \quad 29 \mathrm{JuI} 31$

No returns

No returns

$4139 \quad 7000 \quad 4$ Aug 31

44746624 Aug 31

$47 \begin{array}{lllll}15 & 7200 & 7 \mathrm{JuI} & 31\end{array}$

$4129 \quad 7110 \quad 14$ Aug 31

$47316944 \quad 30$ iay 31

$4102 \quad 7156 \quad 12$ JuI 31

$4121 \quad 7030 \quad 7$ Aug 31

$4730 \quad 7106 \quad 14$ Aug 31

$4115 \quad 7200 \quad 20$ JuI 31

$4121 \quad 7027 \quad 28$ Jun 31

4I51 $6958 \quad 7$ Jun 31

4I $55 \quad 6958 \quad 14$ Jun 31

$4057 \quad 7157 \quad 3$ Jul 31

4156957 13 Jun 31

$4744 \quad 7020 \quad 17$ Jun 31

$4154 \quad 6957 \quad 14$ Jun 31

$\begin{array}{lllll}42 & 22 & 71 & 03 & 14 \\ 4 & \text { Jun } 31\end{array}$

$\begin{array}{llll}42 & 05 & 7010 \quad 1 / 4 \\ 11 & 10 n & 31\end{array}$

$11444 \quad 7020 \quad 30$ Jun 31

$4201 \quad 7000 \quad 12$ Jun 31

No returns

No returns

$4133 \quad 7032 \quad 2$ Aug 31

$4121 \quad 7030 \quad 4$ Aug 31

$4054 \quad 7220 \quad 22$ JuI 31

No returns

No returns 
Released

Recovered

\begin{tabular}{|c|c|c|c|c|c|c|}
\hline Bottie & Lat & Long & Date & Iat & Iong & Date \\
\hline 3642 & 4049 & 6740 & 4 Jun & 4703 & 7153 & 22 JuI 31 \\
\hline 3645 & 4049 & 6740 & 4 Jun & $541 ?$ & 1000 & $30 \operatorname{Oct} 32$ \\
\hline 3655 & 4116 & 6754 & $4 \mathrm{Jun}$ & 4121 & 7040 & 12 Jul 31 \\
\hline$3 6 6 \longdiv { 4 }$ & 4129 & 6720 & 4 Jun & 4117 & 7016 & 4 Sep 31 \\
\hline 3666 & 4129 & 6720 & 4 Jun & 4727 & 7036 & $13 \mathrm{Aug} 31$ \\
\hline 3668 & 4129 & 6720 & 4 Jun & 4117 & 7016 & 15 JuI 31 \\
\hline 3670 & 4729 & 6720 & 4 Jun & 4121 & 7030 & 27 Jul 31 \\
\hline 3672 & 4704 & 6702 & 4 Jun & 4119 & $7 0 \longdiv { 4 8 }$ & 24 JuI 31 \\
\hline 3673 & 4104 & 6702 & 4 Jun & 4115 & 7006 & 21 Jul 31 \\
\hline & 4109 & 6616 & 5 Jun & \multicolumn{3}{|c|}{ No returns } \\
\hline 3695 & 4118 & 6626 & 5 Jun & 4117 & 6958 & 27 Aug 31 \\
\hline 3698 & 4718 & 6626 & 5 Jun & 4127 & 7033 & 12 Aug 31 \\
\hline 3699 & 4178 & 6626 & 5 Jun & 5523 & 0725 & 20 sep 32 \\
\hline 3700 & 4118 & 6626 & 5 Jun & 4723 & 7031 & 12 Aug 31 \\
\hline 3701 & 4144 & 6644 & 5 Jun & 4121 & 7042 & 23 JuI 37 \\
\hline 3702 & 4144 & 6644 & 5 Jun & 4727 & 7055.5 & 30 JuI 31 \\
\hline 3706 & 4144 & $66 \mathrm{LH}_{4}$ & 5 Jun & 4135 & 7039 & $30 \mathrm{JuI} 31$ \\
\hline 3707 & 4144 & 6644 & 5 Jun & 4116 & 7011 & 29 Jul 31 \\
\hline 3712 & 4140 & 6557 & 5 Jun & 4130 & 7106 & 30 Jul 31 \\
\hline 3726 & 4159 & 6610 & 5 Jun & 4121 & 7030 & 5 Aug 31 \\
\hline 3727 & 4759 & 6610 & 5 Jun & 4119 & 7049 & 27 Aug 31 \\
\hline 3734 & 4213 & 6547 & 6 Jun & 4839 & 0413 & 25 May 33 \\
\hline & 4231 & 6608 & $6 \mathrm{Jun}$ & \multicolumn{3}{|c|}{ No returns } \\
\hline 3759 & 4222 & 6629 & 6 Jun & 屿？ & $66 ?$ & 15 Apr 31। \\
\hline 3761 & 4210 & 6704 & 6 Jun & 4452 & 6524 & $90 \cot 32$ \\
\hline 3763 & 4210 & 6704 & 6 Jun & 4452 & 6527 & 13 Oct 31 \\
\hline & 4234 & 6718 & $7 \mathrm{Jun}$ & \multicolumn{3}{|c|}{ No returns } \\
\hline & 4223 & 6754 & 7 Jun & \multicolumn{3}{|c|}{ No returns } \\
\hline 3792 & 4744 & 6813 & 7 Jun & 4430 & 6348 & 17 sep 31 \\
\hline 3798 & 4144 & 6813 & 7 Jun & 4117 & 7014 & 30 Aug 31 \\
\hline & 4210 & 6833 & 7 Jun & \multicolumn{3}{|c|}{ No returns } \\
\hline
\end{tabular}


From 5 to 20 bottles with drags per station and in strings of 2 bottles every 2 miles.

\section{Released}

Bottle Lat Iong Date

$38844 \quad 4105 \quad 7048 \quad 5 \mathrm{Apr}$

$3885 \quad 4705 \quad 7048 \quad 5 \mathrm{Apr}$

$40417041 \quad 5 \mathrm{Apr}$

$4046 \quad 7006 \quad 5 \mathrm{Apr}$

$3908 \quad 4031 \quad 6946 \quad 6$ Apr

$3910 \quad 4031 \quad 6946 \quad 6$ Apr

$4008 \quad 6934 \quad 6 \mathrm{Apr}$

$3957 \quad 6925 \quad 6$ Apr

$40086845 \quad 6$ Apr

$3955 \quad 6835 \quad 6$ Apr

$\begin{array}{lllll}3976 & 4057 \quad 6827 \quad 7 & \mathrm{Apr}\end{array}$

$\begin{array}{lllll}3980 & 4057 & 68 & 27 & 7\end{array} \mathrm{Apr}$

$3984 \quad 4055 \quad 6825 \quad 7 \mathrm{Apr}$

$3989 \quad 4050 \quad 6822.7$ Apr

4010

4037

4038

4039

4051

4084

4099

4103

4705

4109

4711

4175

4117
4035

4044

4022

4023

4018

4045

4045

4045

4130

4110

4104

4030

4708

4143

4243

4743

4724

4051

4052

4149

4149

4149

4158
6812

$\begin{array}{ll}69 & 08\end{array}$

6853

$\begin{array}{ll}68 & 03\end{array}$

6713

6731

6731

6731

6707

6747

6643

6720

6614

6550

6550

65. 50

6624

6645

6607

6643

6643

6643

6621
7 Apr

7 Apr

7 Apr

7 Apr

7 Apr

8 Apr

8 Apr

8 Apr

$8 \mathrm{Apr}$

8 Apr

8 Apr

8 Apr

9 Apr

9 Apr

9 Apr

9 Apr

9 Apr

9 Apr

9 Apr

$10 \mathrm{Apr}$

$10 \mathrm{Apr}$

$10 \mathrm{Apr}$

$10 \mathrm{Apr}$

\section{Recovered}

Lat Long Date

$4121 \quad 7032 \quad 28$ Jun 32

$41 \quad 10 \quad 7133 \quad 21 \mathrm{Hug} 54$

No returns

No returns

$56 \quad 30 \quad 0658 \quad 28$ Aug 33

$5215 \quad 06 \quad 47 \quad 25 \mathrm{Jan} 34$

No returns

No returns

No returns

No returns

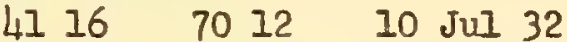

$4114 \quad 7007 \quad 8$ Aug 32

$4116 \quad 7012 \quad 2$ Aug 32 *

$3825 \quad 2820 \quad 8 \mathrm{Feb} 33$ *

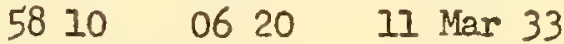

No returns

No returns

No returns

No returns

$56 \quad 30 \quad 0658 \quad 18$ Apr 33

$5408 \quad 1012 \quad 8$ Aug 33

$\begin{array}{llll}4126 & 70 & 53 & 18 \text { Aug } 32\end{array}$

$\begin{array}{lllll}22 & 25 & 74 & 00 & 12 \\ \mathrm{Feb} & 40\end{array}$

No returns

No returns

No returns

$5725 \quad 0725 \quad 7$ Aug 35

$5240 \quad 0940 \quad 21$ Jun 33

$2618 \quad 7700 \quad 27 \operatorname{Mar} 33$

$5355 \quad 1005 \quad 10 \mathrm{Jan} 34$

No returns

No returns

No returns

$\begin{array}{lllll}56 & 30 & 06 \quad 58 & 18 & \text { Apr } 33\end{array}$

$4840 \quad$ OI $30 \quad 5$ Aug 33

$\begin{array}{llll}4717 & 5519 \quad 17 \text { Oct } 32\end{array}$

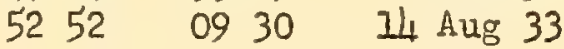




\begin{tabular}{|c|c|c|c|c|c|c|c|}
\hline Bottle & Lat & Long & Date & Lat & Long & Date & \\
\hline 4129 & 4203 & 6609 & $10 \mathrm{Apr}$ & 5508 & 0808 & 3 Oct 33 & $\not$ \\
\hline 4144 & 4210 & 6552.5 & $10 \mathrm{Apr}$ & 4536 & 6447 & 9 Jul 32 & * \\
\hline 4155 & 4216 & 6542 & $10 \mathrm{Apr}$ & 4505 & 6639 & 9 Jul 32 & 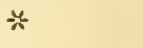 \\
\hline 4156 & 4216 & 6542 & $10 \mathrm{Apr}$ & 7035 & $2355 \mathrm{E}$ & $25 \operatorname{Jan} 34$ & * \\
\hline 4157 & 4217 & 6540 & $10 \mathrm{Apr}$ & 4426.5 & 6736 & 27 Jul 32 & * \\
\hline 4763 & 4220 & 6532.5 & $10 \mathrm{Apr}$ & 6000 & 0130 & 7 Oct 33 & $*$ \\
\hline 4780 & 4227 & 6515 & 11 Apr & 5128 & 0935 & 20 May 33 & 鹿 \\
\hline 4181 & 4228 & 6518 & 11 Apr & 4420 & 6612 & 24 oct 32 & $\ddot{*}$ \\
\hline 4200 & 4238.5 & 6542 & $1 I \mathrm{Apr}$ & 4432 & 6356 & 26 Dec 32 & * \\
\hline 4201 & 4239.5 & 6545.5 & $11 \mathrm{Apr}$ & 4501 & 6650 & 28 Aug 32 & * \\
\hline 4203 & 4241 & 6548 & 11 Apr & 4458 & 6658 & 8 Aug 32 & * \\
\hline 4210 & 4242 & 6556 & $11 \mathrm{Apr}$ & 4708 & 5504 & 20 Sep 32 & * \\
\hline 4218 & 4234 & 6610.5 & $11 \mathrm{Apr}$ & 4354 & 6619 & 12 May 32 & * \\
\hline 4219 & 4232 & 6613 & $11 \mathrm{Apr}$ & 4505 & 6639 & 6 Aug 32 & * \\
\hline 4222 & 4231 & 6631 & $17 \mathrm{Apr}$ & 4329 & 6549 & 29 Apr 32 & * \\
\hline 4232 & $42 \quad 22$ & 6831 & $11 \mathrm{Apr}$ & 4436.5 & 6650.5 & 22 Aug 32 & * \\
\hline 4235 & 4221 & $66 \lcm{40}$ & $17 \mathrm{Apr}$ & 4345 & 6627 & $10 \mathrm{Jun} 32$ & * \\
\hline 4240 & 4220 & $66 \quad 43$ & $11 \mathrm{Apr}$ & 5415 & 0845 & 1 Jun 33 & * \\
\hline 4243 & 4218 & 6648 & $11 \mathrm{Apr}$ & 4507 & 6635 & $7 \mathrm{Juz} 32$ & * \\
\hline 4248 & 4216 & 6653 & $11 \mathrm{Apr}$ & 4456 & 6710 & 17 Sep 32 & $*$ \\
\hline 4259 & 4233 & 6729 & $11 \mathrm{Apr}$ & 5724 & 0723 & 6 Aug 33 & \\
\hline 4265 & 4233 & 6729 & 11 Apr & 450 & 6230 & 21 Aug 32 & \\
\hline 4273 & $42 \quad 22$ & 6800 & $12 \mathrm{Apr}$ & 4120 & 7044 & $10 \mathrm{Jul} 32$ & \\
\hline 4280 & 4222 & 6800 & $12 \mathrm{Apr}$ & 4508 & 6621 & 29 Aug 32 & \\
\hline 4282 & 4222 & 6800 & $12 \mathrm{Apr}$ & 2800 & 1600 & 28 May 34 & \\
\hline 4298 & 4202 & 6746 & $12 \mathrm{Apr}$ & 5330 & 1000 & 30 JuI 33 & \\
\hline 4300 & 4202 & 6746 & $12 \mathrm{Apr}$ & 5457 & 0830 & 22 Jul 33 & \\
\hline 4302 & 4202 & 6746 & $12 \mathrm{Apr}$ & 5850 & $0520 \mathrm{E}$ & 15 oct 33 & \\
\hline & 4217 & 7025 & $15 \mathrm{Apr}$ & No returr & & & \\
\hline & 4207 & 6956 & $15 \mathrm{Apr}$ & No returr & & & \\
\hline 4326 & 4217 & 6923 & $16 \mathrm{Apr}$ & 4756 & 6652 & 18 Jul 32 & At sea \\
\hline & 4755 & 6907 & $16 \mathrm{Apr}$ & No return & & & \\
\hline 4352 & 4750 & 6919 & $16 \mathrm{Apr}$ & 4325 & $0300 \mathrm{E}$ & $24 \operatorname{Mar} 34$ & * \\
\hline 4356 & 4148 & 6924 & $16 \mathrm{Apr}$ & 6600 & $1300 \mathrm{E}$ & $18 \mathrm{Dec} 33$ & \% \\
\hline 4370 & 4142 & 6945 & $16 \mathrm{Apr}$ & 3700 & 2500 & 12 act 33 & $\ddot{*}$ \\
\hline 4373 & 4142 & 6945 & $16 \mathrm{Apr}$ & 4120 & 7044 & 10 JuI 32 & * \\
\hline 4378 & 4737.5 & 6947 & $16 \mathrm{Apr}$ & 4356 & 6001 & $9 \operatorname{Jan} 33$ & * \\
\hline 4381 & 4735 & 6943 & $16 \mathrm{Apr}$ & 4129 & 7045 & $17 \mathrm{Sep} 34$ & * \\
\hline 4397 & 4727 & 6926 & $16 \mathrm{Apr}$ & 4055.5 & $72 \quad 14$ & 8 oct 32 & * \\
\hline 4406 & 4116 & 6919 & $16 \mathrm{Apr}$ & 4732 & 5557 & 26 Dec 32 & * \\
\hline 4412 & 4113 & 6912 & $16 \mathrm{Apr}$ & 5206 & 1030 & 17 Jun 33 & $\approx$ \\
\hline 4419 & 4110.7 & 6904.5 & $16 \mathrm{Apr}$ & 4221 & 7042 & 17 Aug 32 & $\stackrel{\circ}{*}$ \\
\hline 4455 & 4110 & 6840 & $17 \mathrm{Apr}$ & 3655 & 2510 & 9 oct 33 & * \\
\hline 4473 & 4125 & 6849 & $17 \mathrm{Apr}$ & 5515 & 0640 & 11 Nov 33 & * \\
\hline 4487 & 4134 & 6850 & $17 \mathrm{Apr}$ & 3705 & 2510 & 15 Jun 33 & $\ddot{x}$ \\
\hline
\end{tabular}

* Bottles seeded in string, 2 every 2 miles. 


\begin{tabular}{|c|c|c|c|c|c|c|}
\hline Bottle & Lat & Iong & Date & Lat & Long & Date \\
\hline 4499 & 4146 & 6848 & $17 \mathrm{Apr}$ & 4110 & 7055 & 25 Aug 32 : \\
\hline 4504 & 4750 & 6845 & $17 \mathrm{Apr}$ & 5233 & 0958 & 14 oct 33 . \\
\hline 4507 & 4151 & 6844 & $17 \mathrm{Apr}$ & 3655 & 2510 & $4 \operatorname{Dec} 33$ \\
\hline 4509 & 4150 & 6838 & $17 \mathrm{Apr}$ & 5538 & 0612 & 31 Jul 33 \\
\hline 4523 & 4201 & 6834 & $17 \mathrm{Apr}$ & 5908 & 0320 & $27 \operatorname{Mar} 34$ \\
\hline 4547 & 4221 & 6759 & $18 \mathrm{Apr}$ & 4648 & 5625 & 23 Dec 32 \\
\hline 4565 & 4237 & 6810 & $18 \mathrm{Apr}$ & 4422.5 & 6605 & 16 Jun $32:$ \\
\hline 4574 & 4230 & 6939 & $18 \mathrm{Apr}$ & 4347 & 6607.8 & 2 Jum 32 \\
\hline 4600 & 4233 & 7018 & $18 \mathrm{Apr}$ & 4520 & 6447 & 12 Sep 32 \\
\hline 4602 & 4228.3 & 7024.8 & $18 \mathrm{Apr}$ & Wh 25 & 6408 & $40 \operatorname{ct} 32$ \\
\hline 4611 & 4233 & 7034 & $18 \mathrm{Apr}$ & 4105 & & $32 \operatorname{Mar} 33 *$ \\
\hline 4612 & 4233.3 & 7036.4 & $18 \mathrm{Apr}$ & 4201 & 7012 & 11 May 32 \\
\hline 4613 & 4233 & 7036.4 & $18 \mathrm{Apr}$ & 4122 & 7000 & 31 May $32 *$ \\
\hline 160 & 4233.9 & 7039.4 & $18 \mathrm{Apr}$ & 4201 & 7012 & 24 Apr 32 \\
\hline
\end{tabular}

* Bottles seeded in string, 2 every 2 miles. 
Five to 10 bottles wi th drags released at each station and in strings of 2 bottles every 2 miles.

\begin{tabular}{|c|c|c|c|c|c|c|}
\hline \multicolumn{4}{|c|}{ Released } & \multicolumn{3}{|c|}{ Recovered } \\
\hline Bottle & Lat & Long & Date & Lat & Long & Date \\
\hline 4636 & 4139.5 & 6950 & 19 Jun & 4739.4 & 6959 & 18 Aug 3 \\
\hline 4639 & 4140 & $6 9 \longdiv { 4 9 }$ & 19 Jun & 4727 & 7119 & 22 JuI 33 \\
\hline 4649 & 4143 & 6945.5 & $19 \mathrm{Jun}$ & 3516 & 7531 & 27 oct 33 \\
\hline 4652 & 4746 & 6941 & ฉD Jun & 4442 & 6546 & $13 \mathrm{Sep} 33$ \\
\hline 4656 & 4750 & 6933 & 20 Jun & 2130 & 7110 & $9 \mathrm{Jan} 37$ \\
\hline 4706 & 4204 & 6749 & 21 Jun & 4327 & 6528 & 18 Aug 33 \\
\hline 4713 & 4227 & 6803 & 20 Jun & 4434 & 6722 & $9 \operatorname{Mar} 3$ \\
\hline 4716 & 4222 & 6801 & $20 \mathrm{Jun}$ & 4437 & 6650 & 31 Jul 3 \\
\hline 4718 & 4220 & 6759 & $20 \mathrm{Jun}$ & 4415.5 & 6621 & 2 Aug 3 \\
\hline 4721 & 4217 & 6759 & 20 Jun & 3540 & 7529 & 26 oct 33 \\
\hline 4722 & 4213.5 & 6755 & 21 Jun & 472 & $70 \quad 46$ & 19 Sep 33 \\
\hline 4723 & 4213.5 & 6755 & 21 Jun & 3745 & 2545 & 15 Jun 3 \\
\hline 4724 & $\begin{array}{l}4210 \\
1222\end{array}$ & $\begin{array}{ll}67 & 52.5 \\
67 & 30\end{array}$ & 21 Jun & $\begin{array}{ll}45 & 10 \\
\text { No retur }\end{array}$ & $\begin{array}{l}6620 \\
\text { ns }\end{array}$ & 7 Sep 3 \\
\hline 4741 & 4231 & 6723 & 21 Jun & 4340 & 6603 & 2 Apr 34 \\
\hline 4743 & 4231 & 6723 & $2 I$ Jun & 4425 & 6612 & I Sep 33 \\
\hline 4744 & 4231 & 6723 & 21 Jun & 4420 & 6608 & $13 \mathrm{Sep} 3$ \\
\hline 4751 & 4210 & 6704 & 27 Jun & 4730 & 6705 & 6 Ju2 33 \\
\hline 4754 & 4210 & 6704 & 21 Jun & 4503 & 6504 & 28 oct 33 \\
\hline 4762 & 4225 & 6644 & 22 Jun & 4742 & 0321 & 18 Jun 35 \\
\hline 4768 & 4207 & 6619 & 22 Jun & W4 16 & 6622 & $15 \mathrm{Nov}$ \\
\hline 4770 & 4210 & 6616 & 22 Jun & 4354 & 6445 & 27 Aug 33 \\
\hline 4791 & 4223.5 & 6553 & 22. Jun & 4315 & 6545 & 1 Aug 33 \\
\hline 4798 & 4229.5 & 6549 & 22 Jun & 4452 & 6527 & 22 oct 33 \\
\hline 4800 & 4231 & 6548.5 & 22 Jun & 4458 & 6704 & 28 Aug 33 \\
\hline 4801 & 4231 & 6548.5 & 22 Jun & 4205 & 7009 & $210 \operatorname{lot} 33$ \\
\hline 4802 & 4232 & 6548 & 23 Jun & 4416 & 6608 & $23 \mathrm{JUI} 33$ \\
\hline 4804 & 4233.5 & 6547.5 & 22 Jun & 4452 & 6527 & 1 Aug 3 \\
\hline 4812 & 4237 & 6553.5 & 22 Jun & 4434 & 6555 & 21 Aug 33 \\
\hline 4814 & 4237 & 6553.5 & 22 Jun & 4800 & 0400 & If $\operatorname{Jan} 35$ \\
\hline 4819 & $42 \quad 40$ & 6602 & 22 Jun & 3840 & 2715 & 11 May 34 \\
\hline 4824 & 4246 & 6557 & 22 Jun & 4330 & 6530 & 21 Aug 33 \\
\hline 4826 & 4248 & $\begin{array}{l}6555 \\
6553\end{array}$ & 22 Jun & 4305 & $\begin{array}{l}6600 \\
66 ? 8\end{array}$ & $\begin{array}{l}21 \text { Jul } 33 \\
25 \text { Nov } 33\end{array}$ \\
\hline & 4252 & 6552 & 23 Jun & 4420 & 6612 & 4 Nor 33 \\
\hline & 4255 & 6551 & $23 \mathrm{Jun}$ & 4220 & 6650 & 10 Aug 33 \\
\hline
\end{tabular}




\begin{tabular}{|c|c|c|c|c|c|c|c|}
\hline Bottle & Lat & Long & Date & Lat & Long & Date & \\
\hline 4838 & 4301 & 6549 & 23 Jun & 4417 & 6618 & 1 Aug 33 & * \\
\hline 4839 & 4301 & 6549 & 23 Jun & 5128 & 0923 & 31 Jan 37 & * \\
\hline 4841 & 4303 & 6548 & 23 Jun & 4433 & 6602 & 25 JuI 33 & $\ddot{*}$ \\
\hline 4842 & 4305 & 6547 & 23 Jun & 4440.5 & 6548.5 & I4 Nov 33 & * \\
\hline 4846 & 4309 & 6545.5 & 23 Jun & 4430 & 6606 & 16 oct 33 & $*$ \\
\hline 4850 & 4315 & 6543 & 23 Jun & 4426 & 6600 & 7 Sep 33 & $\ddot{*}$ \\
\hline 4856 & 4318.5 & 6537.5 & 23 Jun & 4523 & 6439 & 21 Aug 33 & $\ddot{*}$ \\
\hline 4857 & 4318.5 & 6537.5 & 23 Jun & 4436 & 6558 & 13 Sep 33 & is \\
\hline 4858 & 4218 & 6530 & $23 \mathrm{Jun}$ & 4502 & 6649 & 23 Aug 33 & * \\
\hline 4862 & 4214 & 6531 & 23 Jun & 4456.5 & 6517 & 31 JuI 33 & $\ddot{H}$ \\
\hline 4866 & 4210 & 6531 & 23 Jun & 4450 & 6659 & 27 Aug 33 & $\ddot{*}$ \\
\hline 4867 & 4210 & 6531 & 23 Jun & 4317 & $6 5 \longdiv { 4 6 }$ & 21 JuI 33 & $\ddot{x}$ \\
\hline 4868 & 4208 & 6532 & $23 \mathrm{Jun}$ & 4416 & 6608 & 23 Jul 33 & $\ddot{*}$ \\
\hline 4870 & 4208 & 6532 & 23 Jun & 2915 & 1327 & 12 Aug 35 & $\ddot{x}$ \\
\hline 4876 & 4200 & 6532 & 23 Jun & 4322 & 6540 & 4 Aug 33 & * \\
\hline 4877 & 4200 & 6532 & 23 Jun & 3745 & 2545 & 11 Apr 35 & * \\
\hline 4896 & 4158.3 & 6546.5 & 24 Jun & 4337 & 6516 & 27 Aug 33 & H \\
\hline \multirow{2}{*}{4912} & 4132 & 6555 & 24 Jun & 4046 & 7245 & 26 Aug 33 & \\
\hline & 4139 & 6608 & $24 \mathrm{Jun}$ & \multicolumn{4}{|c|}{ No returns } \\
\hline 4926 & 4139 & 6646 & 24 Jun & 4339 & 6508 & 28 Sep 33 & \\
\hline 4927 & 4139 & 6646 & $24 \mathrm{Jun}$ & 4205 & 6655 & 1 Sep 33 & \\
\hline 4936 & 4130 & 6707 & 25 Jun & 4135 & 7051 & 22 Aug 33 & \\
\hline \multirow[t]{4}{*}{4944} & 4119 & 6639 & 25 Jun & 4131 & 7119 & 1 Sep 33 & \\
\hline & 4103 & 6625 & 25 Jun & \multicolumn{4}{|c|}{ No returns } \\
\hline & 4048 & 6644 & 25 Jun & \multicolumn{4}{|c|}{ No returns } \\
\hline & 4053 & 6621 & 25 Jun & \multicolumn{4}{|c|}{ No retarns } \\
\hline 4986 & 4056 & 6659 & 26 Jun & 4047 & 7308 & 24 Aug 33 & \\
\hline 4988 & 4056 & 6659 & 26 Jun & 4047.5 & 7239 & 24 Aug 33 & \\
\hline 4990 & 4056 & 6659 & 26 Jun & 4116 & 7011 & 1 Aug 33 & \\
\hline 4992 & 4056 & 6659 & 26 Jun & 4115 & 7010 & 31 Jนป 33 & \\
\hline 5004 & 4038 & 6752 & 26 Jun & 4120.5 & $7049 \cdot 5$ & 8 Aug 33 & \\
\hline 5006 & 4038 & 6752 & $26 \mathrm{Jun}$ & 4111 & 7055 & 25 Aug 33 & At sea \\
\hline \multirow{3}{*}{5010} & 4038 & 6752 & 26 Jun & 4050 & 7230 & 23 Aug 33 & \\
\hline & 4105 & 6735 & 26 Jun & \multicolumn{4}{|c|}{ No returns } \\
\hline & 4020 & 6800 & 26 Jun & \multicolumn{4}{|c|}{ No returns } \\
\hline 5024 & 4008 & 6814 & 27 Jun & 3726 & 7540 & 6 Sep 33 & \\
\hline 5030 & 4008 & 6814 & 27 Jun & 3640 & 7555 & $4 \operatorname{Jan} 34$ & \\
\hline 5040 & 4017.5 & 6835.5 & 27 Jun & 4013 & 6847 & 27 Jun 33 & At sea * \\
\hline 5046 & 4023 & 6845 & 27 Jun & 3616 & 7547 & $29 \operatorname{Oct} 33$ & \\
\hline 5054 & 4028.5 & 6852 & 27 Jun & 4035 & 7340 & 24 Aug 33 & \\
\hline 5056 & 4030.5 & 6853.5 & 27 Jun & 4036 & 7330 & 4 Sep 33 & $*$ \\
\hline
\end{tabular}




\begin{tabular}{|c|c|c|c|c|c|c|}
\hline Bottle & Lat & Long & Date & Lat & Iong & Date \\
\hline 5058 & 4032 & 6856 & 27 Jun & 4120 & $70 \quad 02$ & 4 Sep $33 *$ \\
\hline 5070 & $4 0 \longdiv { 4 3 }$ & 6905 & 27 Jun. & 4128 & 7110 & 17 Aug 33 \\
\hline 5072 & $4 0 4 \longdiv { 4 }$ & 6905.5 & 27 Jun & 4225 & 6550 & 25 Jul 33 \\
\hline 5079 & 40 兵 & 6905 & 27 Jun & 4036 & 7326 & 25 Ang 33 \\
\hline 5087 & 4059 & 6904 & 27 Jun & 4114 & 6845 & 16 Aug 33 \\
\hline 5090 & 4103 & 6904 & 27 Jun & 4430 & 6600 & 11 Sep 33 \\
\hline 5096 & 4709 & 6904 & 27 Jun & 4121 & 7030 & 31 JuI 33 \\
\hline 5098 & 4109 & 6904 & 27 Jun & 4048 & 7237 & 24 Aug 33 \\
\hline 5099 & 4109 & 6904 & 27 Jun & 5000 & 0515 & 26 Dec 34 \\
\hline 5102 & 4709 & 6904 & 27 Jun & 4058 & 7208 & 24 Aug 33 \\
\hline \multirow[t]{3}{*}{5111} & 4725 & 6836 & 27 Jun & 4433 & 6602 & 21 oct 33 \\
\hline & 4200 & 6822 & 28 Jun & No retur & & \\
\hline & 4745 & 6815 & 28 Jun & No retur & & \\
\hline 5138 & 4150 & 6907 & $28 \mathrm{Jun}$ & 4417.5 & 6608 & 12 Sep 33 \\
\hline 5140 & 4150 & 6907 & 28 Jun & 4400 & 6625 & $19 \operatorname{Oct} 33$ \\
\hline 5147 & 4138 & 6920 & 28 Jun & 4520.5 & 6447 & 19 Oct 33 \\
\hline 定49 & 4138 & 6920 & 28 Jun & 3655 & 2510 & 18 Nov 34 \\
\hline 5154 & 4733 & 6938 & 28 Jun & 4130 & 7105 & $22 \mathrm{JuI} 33$ \\
\hline 5.56 & 4733 & 6938 & 28 Jun & 4129 & 7109 & $23 \mathrm{Jul} 33$ \\
\hline 5157 & 4133 & 6938 & 28 Jun & 3655 & 2510 & 7 Nov 34 \\
\hline 5158 & 4133 & 6938 & 28 Jun & 4729 & 7109 & 23 Ju? 33 \\
\hline 5159 & 4133 & 6938 & 28 Jun & 4054 & 7220 & 26 Aug 33 \\
\hline 5160 & 4733 & 6938 & 28 Jun & 4724 & 7057 & 24 JuI 33 \\
\hline 5164 & 4135 & 6951 & 23 Jun & 4120 & 7000 & 5 JuI 33 th \\
\hline 5166 & 4132 & 6959 & 28 Jun & 4118 & 6958.5 & 5 JuI 33 \\
\hline
\end{tabular}

* Bottles seeded in string, 2 every 2 miles. 
Five to 15 bottles released at stations. Others in strings of 2 bottles every 2 miles. Odd numbers wi th drags, even numbers wi thout drags.

Released

Bottle Lat Long Date

$5173 \quad 4742 \quad 6949 \quad 27$ Apr

$5178 \quad 4745 \quad 6941 \quad 27$ Apr

5180

5187

5189

5200

5202

5223

5231

5233

5235

5236

5239

5242

5244

5264

5270

5272

5280

5287

5293

5306

5309

5328

5332

5335

5336

5337

5339

5342

5344

5346

5347

5349

5358
4746

4748

4749

4153

4754

4749

4750

4752

4200

4200

4200

4204

4204

4204

4208

6939

6932

27 Apr

6929

$69 \cdot 15$

27 Apr

27 Apr

6913

6823

27 Apr

$\begin{array}{lll}68 & 17\end{array}$

27 Apr

6812

23 Apr

28 Apr

6820

$28 \mathrm{Apr}$

28 Apr

6820

$28 \mathrm{Apr}$

6820

$28 \mathrm{Apr}$

6828

$28 \mathrm{Apr}$

6828

28 Apr

6828

6746

4204

4157

4757

4218

4215

41.57

4157

4206

4212

4212

4212

4212

4212

4216

4216

4216

4216

4216

$42 \quad 23$
6743

6736

6736

6706

6704

6653

6653

6640

6620

6620

6620

6620

6620

6624

6624

6624

$66 \quad 24$

$\begin{array}{ll}66 & 17\end{array}$

6617
$28 \mathrm{Apr}$

29 Apr

29 Apr

29 Apr

29 Apr

29 Apr

29 Apr

$30 \mathrm{Apr}$

30 Apr

$30 \mathrm{Apr}$

$30 \mathrm{Apr}$

30 Apr

30 Apr

30 Apr

$30 \mathrm{Apr}$

$30 \mathrm{Apr}$

30 Apr

30 Apr

30 Apr

30 Apr

30 Apr
Recovered

Lat Long Date

$38350855 \quad 23$ Feb 36 *

$2420 \quad 7745 \quad 15$ Dec $37 \%$

322064446 Jan $35 *$

$4039 \quad 73 \quad 17 \quad 19$ Sep 34 *

$4101720023 \operatorname{Sep} 34 *$

$4422.56752 .5 \quad 5$ Oct $35 *$

$4342 \quad 6950$ II Aug $34 *$

$50250505 \quad 29$ JuI 35 *

No returns

山4 0566 I0 3 Sep $34 *$

$\begin{array}{llllll}40 & 09 & 74 & 01.5 & 14 & \text { Sep } 34\end{array}$

$5300 \quad 1000 \quad 5$ JuI 35

$4252.57049 \quad 16$ Sep 34

$4450 \quad 6530 \quad 18$ JuI 34

$\begin{array}{lllllll}44 & 02 & 68 & 36 & 19 & \mathrm{JuI} & 34\end{array}$

$5300 \quad 1000 \quad 23$ Aug 35

$4424 \quad 6612 \quad 30$ Jun $34 *$

$4350 \quad 6618 \quad 30$ Jun $34 *$

$4355 \quad 6913 \quad 11$ Aug 34 *

$58550310 \quad 3$ Dec 35 *

452064426 Aug $34 *$

$5500 \quad 0835 \quad 27$ Mar 35*

$43376516 \quad 27$ May 34 *

$5630 \quad 0658 \quad 4$ Sep $35 *$

$4316 \quad 6420 \quad 24$ May 34

$\begin{array}{lllll}4322 & 65 & 30 & 24 \\ \text { May } 34\end{array}$

$41336959 \quad 6$ oct 34

$\begin{array}{lll}43 & 6521 & 23 \\ \text { May } 34\end{array}$

$2130 \quad 7130 \quad 26$ Nov 41

$4416 \quad 6622 \quad 3$ Jul 34

L4 $30.56555 \quad 5$ JuI 34

$4304 \quad 6600 \quad 18$ Jun 34

$4447 \quad 6645 \quad 2$ Aug 34

$4452 \quad 6527 \quad 23 \mathrm{JuI} 34$

$4345 \quad 6919 \quad 30$ oct 34

$\begin{array}{llll}4521 & 64 & 15 & 15 \mathrm{Aug} 34\end{array}$

* Bottles seeded in string, 2 every 2 miles. 


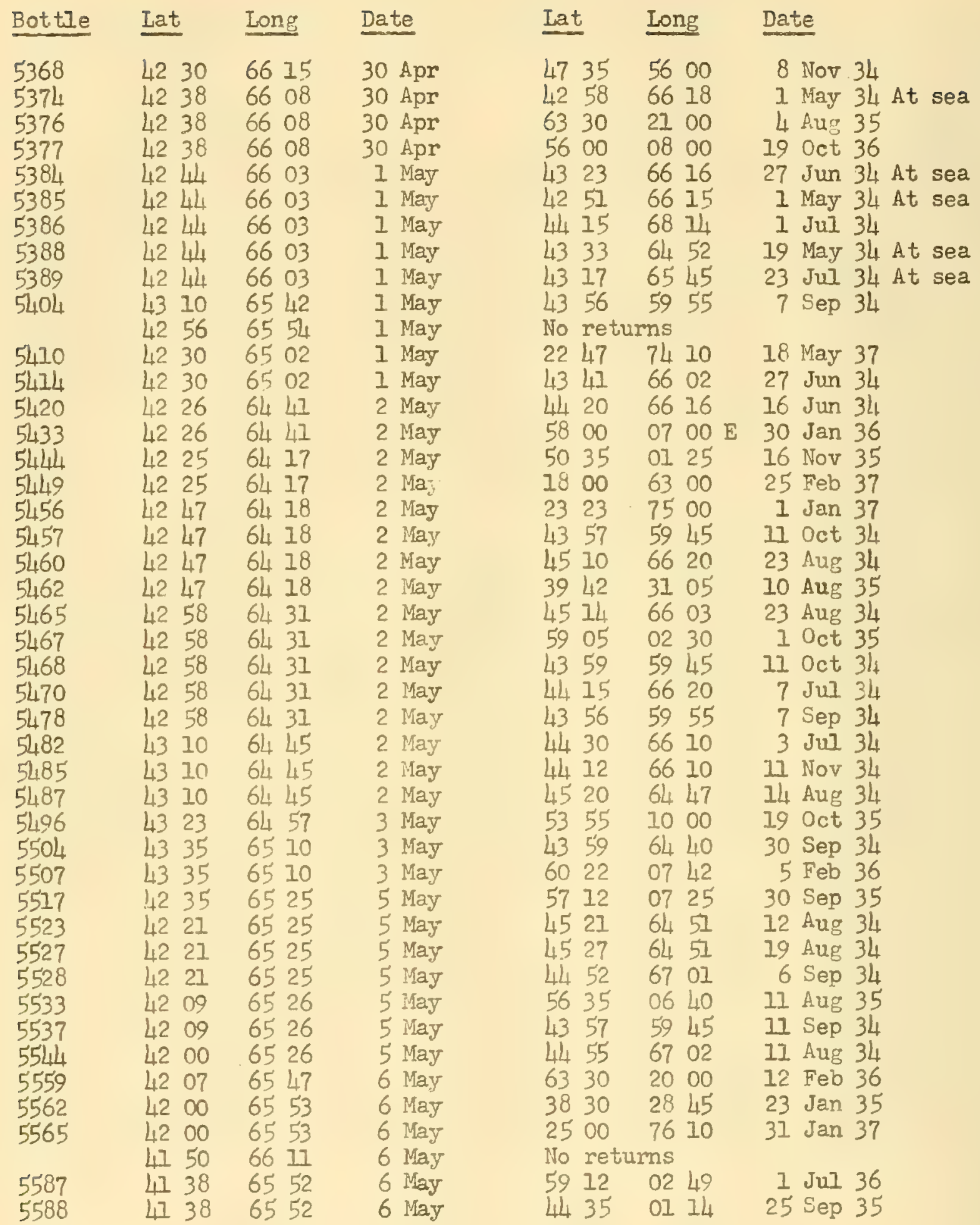


Released

Recovered

\begin{tabular}{|c|c|c|c|c|c|c|}
\hline Bottle & Lat & Long & Date & Lat & Long & Date \\
\hline 5604 & 4113 & 6616 & 8 May & 5500 & 0830 & 12 Apr $35^{\circ}$ \\
\hline 5610 & 4120 & 6632 & 8 May & 5407 & 1005 & $11 \mathrm{JuI} 35$ \\
\hline 5617 & 4131 & $6 6 \longdiv { 4 5 }$ & 8 May & 2330 & 7545 & $5 \mathrm{Jan} 38$ \\
\hline 5620 & 4131 & 6645 & 8 May & 4721 & 7036 & 24 JuI 34 \\
\hline \multirow[t]{2}{*}{5621} & 4133 & 6645 & 8 May & 3941 & & 4 Sep 34 \\
\hline & 4727 & 6718 & 9 May & \multicolumn{3}{|c|}{ No returns } \\
\hline 5649 & L] 01 & 6703 & 9 May & 5535 & 0620 & 1 Sep 35 \\
\hline \multirow[t]{2}{*}{5653} & 4101 & 6703 & 9 May & \multirow{2}{*}{\multicolumn{3}{|c|}{ No returns }} \\
\hline & 4036 & 6705 & 10 May & & & \\
\hline \multirow[t]{2}{*}{5669} & 4049 & 6736 & 10 May & 3807 & 7511 & 22 Oct 3 \\
\hline & 4059 & 6746 & 10 May & \multicolumn{3}{|c|}{ No returns } \\
\hline 5693 & 4109 & 6757 & $10 \mathrm{May}$ & 6900 & $1500 \mathrm{E}$ & $6 \operatorname{Mar} 3$ \\
\hline 5701 & 4057 & 6827 & 10 May & 3840 & 2715 & 1 Aug \\
\hline 5710 & 4047 & 6900 & 10 May & 4425 & 6603 & 19 JuI 3 \\
\hline 5715 & 4047 & 6900 & 10 May & 4400 & 6610 & 8 Nov 3 \\
\hline 5727 & 4030 & 6840 & II May & 5145 & 1007 & 11 Apr \\
\hline 5737 & 4030 & 6840 & $11 \mathrm{May}$ & 3630 & 7552 & $25 \mathrm{Sep}$ \\
\hline 5742 & 4105 & 6918 & II May & 4051 & 7236 & 27 Sep 3 \\
\hline 5744 & 4705 & 6918 & 11 May & $40 \lcm{47}$ & 7300 & 21 Sep \\
\hline \multirow[t]{3}{*}{5747} & 4105 & 6918 & 11 May & 4038 & $73 \quad 14$ & $18 \mathrm{Sep} 3$ \\
\hline & 4113 & 6904 & 12 May & \multicolumn{3}{|c|}{ No returns } \\
\hline & 4142 & $68 \quad 32$ & 12 May & \multicolumn{3}{|c|}{ No returns } \\
\hline 5761 & 4120 & 6840 & 12 May & 4516 & 6549 & 23 Aug \\
\hline 5762 & 4120 & 6840 & 12 May & 4530 & 6056 & $10 \mathrm{Sep}$ \\
\hline 5768 & 4720 & 6840 & 12 May & 4452 & 6527 & 21 Jul \\
\hline 5772 & 4734 & 6826 & 12 May & 4425 & 6602 & $4 \mathrm{JuI}$ \\
\hline 5790 & 4125 & 6928 & 13 May & 5415 & 0830 & 27 Jul \\
\hline 5798 & 4125 & 6928 & 13 May & 4049 & 7230 & 13 Oct 34 \\
\hline
\end{tabular}


12 bottles released at each station for all cruises below. $N=$ No ballast $\quad B=$ Ballasted with sand

\begin{tabular}{|c|c|c|c|c|c|c|c|}
\hline \multirow[b]{2}{*}{ Station } & \multicolumn{4}{|c|}{ Released } & \multicolumn{3}{|c|}{ Recovered } \\
\hline & Bottle & Lat & Long & Date & Lat & Long & Date \\
\hline 1 & $12102 \mathrm{~N}$ & 4101 & 7101 & 19 Mar & 4119.8 & 7144 & 23 May 53 \\
\hline 1 & $12107 \mathrm{~B}$ & 4101 & 7101 & 19 Mar & 3524 & 7600 & 8 Aug 56 \\
\hline 2 & & 4031 & 7101 & $19 \mathrm{Mar}$ & No $x$ & returns & \\
\hline 3 & & 4000 & 7100 & 19 Mar & No $x$ & returns & \\
\hline 4 & & 4000 & 6957 & 20 Mar & No $\mathrm{r}$ & returns & \\
\hline 5 & & 3959 & 6900 & 20 Mar & No 1 & returns & \\
\hline 6 & & 4001 & 6827 & 20 Mar & No $\mathrm{r}$ & returns & \\
\hline 7 & & 4027 & 6803 & 20 Mar & No $r$ & returns & \\
\hline 8 & & 4100 & 6731 & $20 \mathrm{Mar}$ & No $\mathrm{r}$ & returns & \\
\hline 9 & & 4118 & 6715 & 21 Mar & No & returns & \\
\hline 10 & & 4146 & 6712 & 21 Mar & No $\mathrm{r}$ & returns & \\
\hline 11 & & 4214 & $67 \mathrm{IL}_{4}$ & 21 Mar & No $r$ & returns & \\
\hline 12 & & 4301 & 6716 & 21 liar & No $\mathrm{I}$ & returns & \\
\hline 13 & $12252 \mathrm{~N}$ & 4340 & 6713 & 21 Mar & 4475 & 6841 & 30 May 53 \\
\hline 14 & $12268 \mathrm{~N}$ & 4338 & 6859 & 22 Mar & 4332.5 & 7014 & 8 Jun 53 \\
\hline 15 & $12273 B$ & 4330 & 6934 & 22 Mar & 4148.3 & 6959.3 & $20 \mathrm{Dec} 54$ \\
\hline 15 & $12275 \mathrm{~B}$ & 4330 & 6934 & 22 Mar & 4317 & 7034 & 24 Apr 53 \\
\hline 15 & $12276 \mathrm{~N}$ & 4330 & 6934 & 22 Mar & 4348.5 & 6940.5 & $18 \mathrm{Apr} 53$ \\
\hline 15 & $12280 \mathrm{~N}$ & 4330 & 6934 & 22 Mar & 4347 & 6940 & $29 \operatorname{Mar} 53$ \\
\hline 16 & $12281 \mathrm{~B}$ & 4301 & 7031 & 22 Mar & 4217 & 7052 & 3 Apr 53 \\
\hline 16 & $12283 \mathrm{~B}$ & 4301 & 7031 & 22 Mar & 4759.5 & 7004.8 & $18 \mathrm{Apr} 53$ \\
\hline 16 & $12286 \mathrm{~N}$ & 4301 & 7031 & $\therefore$ Mar & $42 \quad 56.5$ & 7047.6 & $28 \operatorname{Mar} 53$ \\
\hline 16 & $12288 \mathrm{~N}$ & 4301 & 7031 & $22 \mathrm{Mar}$ & 4256.5 & 7047.6 & 9 Apr 53 \\
\hline 16 & $12292 \mathrm{~B}$ & 4301 & 7031 & $22 \mathrm{Mar}$ & 4254 & 7048.7 & 1 Apr 53 \\
\hline 17 & $12296 \mathrm{~N}$ & 4300 & 7000 & 22 Mar & 4239 & 7041 & 4 Apr 53 \\
\hline 17 & $12297 \mathrm{~B}$ & 4300 & 7000 & 2? Mar & 4144.3 & $70 \quad 37.3$ & 20 Apr 53 \\
\hline 17 & $12300 \mathrm{~N}$ & 4300 & 7000 & 22 Mar & 4239 & $70 \angle 1$ & $2 \mathrm{Apr} 53$ \\
\hline 17 & $12302 \mathrm{~N}$ & 4300 & 7000 & 22 Mar & 4230 & 9050.5 & 6 Apr 53 \\
\hline 17 & $12303 \mathrm{~B}$ & 4300 & 7000 & 22 Mar & 4127 & 7056 & 7 Aug 53 \\
\hline 18 & & 4300 & 6900 & $22 \mathrm{Mar}$ & No & returns & \\
\hline 19 & & 4300 & 6800 & 22 Mar & No $\mathrm{Y}$ & returns & \\
\hline
\end{tabular}




\begin{tabular}{|c|c|c|c|c|c|c|c|}
\hline Station & Bottle & Lat & Long & Date & Lat & Iong & Date \\
\hline 20 & $12335 B$ & 4301 & 6701 & $23 \mathrm{Mar}$ & 4518.5 & 6447.5 & 19 Apr 55 \\
\hline 21 & $12341 \mathrm{~B}$ & 4301 & 6600 & 23 Mar & 4431 & 6554.5 & $17 \mathrm{JuI} 53$ \\
\hline 21 & $12342 \mathrm{~N}$ & 4301 & 6600 & 23 Mar & 4423.2 & 6604 & 16 Sep 53 \\
\hline 21 & $12346 \mathrm{~N}$ & 4301 & 6600 & $23 \mathrm{Mar}$ & 4350 & 6930 & 21 Dec 53 \\
\hline 22 & $12354 \mathrm{~N}$ & 4259 & 6500 & 23 Mar & 4438.3 & 6553 & 29 JuI 53 \\
\hline 22 & $12357 \mathrm{~B}$ & 4259 & 6500 & $23 \mathrm{Mar}$ & 5310 & 0916 & $21 \mathrm{Dec} 54$ \\
\hline 22 & $12358 \mathrm{~N}$ & 4259 & 6500 & $23 \mathrm{Mar}$ & 4149 & 6957 & 7 Nov 53 \\
\hline 22 & $12359 \mathrm{~B}$ & 4259 & 6500 & 23 Mar & 4440 & $6 6 \longdiv { 4 5 . 3 }$ & 6 oct 53 \\
\hline 23 & $12366 \mathrm{~N}$ & 4215 & 6457 & 23 Mar & 4423.2 & 6604 & 30 Apr 53 \\
\hline 24 & $12378 \mathrm{~N}$ & 4210 & 6602 & $23 \mathrm{Mar}$ & 4504 & 6628 & 29 Ju 53 \\
\hline 25 & $12398 \mathrm{~N}$ & 4216 & 6701 & $24 \mathrm{Mar}$ & 4514.5 & 6605 & 16 Sep 53 \\
\hline 26 & & 4218 & 6802 & 24 Mar & No & eturns & \\
\hline 27 & & 4216 & 6900 & 24 Mar & No & eturns & \\
\hline 28 & $12425 B$ & 4214 & 6952 & 24 Mar & 4723.5 & 7003 & 15 Aug 53 \\
\hline 28 & $12428 \mathrm{~N}$ & 4214 & 6952 & $24 \mathrm{Mar}$ & 4204.2 & 7014.4 & 3 Apr 53 \\
\hline 28 & $12430 \mathrm{~N}$ & 4214 & 6952 & 24 Mar & 4205 & 7012.4 & 24 May 53 \\
\hline 28 & $12432 \mathrm{~N}$ & 4214 & 6952 & $24 \mathrm{Mar}$ & 4201.9 & 7011.3 & 5 Apr 53 \\
\hline 28 & $12434 N$ & 4214 & 6952 & 24 Mar & 4204.6 & 7009.4 & 31 Mar 53 \\
\hline 28 & $12436 \mathrm{~N}$ & $42 \quad 14$ & 6952 & $24 \mathrm{Mar}$ & 4204.9 & 7011.3 & 31 Mar 53 \\
\hline 29 & & 4215 & 7002 & $25 \mathrm{Mar}$ & No & eturns & \\
\hline 30 & $12452 \mathrm{~N}$ & 4147 & 6944.5 & $25 \mathrm{Mar}$ & 4757.1 & 6959.2 & $28 \operatorname{Mar} 53$ \\
\hline 30 & $12456 \mathrm{~N}$ & 474 ? & 6944.5 & $25 \mathrm{Mar}$ & 4157.1 & 6959.2 & $28 \operatorname{Mar} 53$ \\
\hline 30 & $12460 \mathrm{~N}$ & 4747 & 6944.5 & $25 \mathrm{Mar}$ & 4759.6 & 7000.9 & 2 Apr 53 \\
\hline 31 & & 4146 & 6900 & 25 Mar & No & eturns & \\
\hline 32 & & 4747 & 6830 & $25 \mathrm{Mar}$ & No & eturns & \\
\hline 33 & & 4155 & 6800 & $25 \mathrm{Mar}$ & No & etums & \\
\hline 34 & & 4747 & 6658 & 27 Mar & No & eturns & \\
\hline 35 & & 4146 & $660 ?$ & 27 Mar & No & eturns & \\
\hline 36 & & 4143 & 6545 & 27 Mar & No & eturns & \\
\hline 37 & & 4119 & 6611.5 & 27 Mar & No & eturns & \\
\hline 38 & & 4117 & 6700 & 27 Mar & No & etums & \\
\hline 39 & $12561 \mathrm{~B}$ & 4115 & 6801 & $28 \mathrm{Mar}$ & 3546.5 & 7518 & $5 \mathrm{Jan} 55$ \\
\hline 40 & & 4116 & 6859 & 28 Mar & No & e turns & \\
\hline 41 & & 4177.5 & 6919.5 & $28 \mathrm{Mar}$ & No & eturns & \\
\hline 42 & & 4035 & 6851 & 29 Mar & No & eturns & \\
\hline 43 & & $4 0 \longdiv { 4 6 }$ & 6759 & $29 \mathrm{Mar}$ & No & eturns & \\
\hline 44 & & 4047 & 6730 & 29 Mar & No & eturns & \\
\hline 45 & & 4046 & 6700 & $29 \mathrm{Mar}$ & No & eturns & \\
\hline 46 & & 4045 & 6633.5 & 29 Mar & No & eturns & \\
\hline 47 & & 4131 & 6629 & 29 Mar & No & eturns & \\
\hline 48 & & 4215 & 6616 & $30 \mathrm{Mar}$ & No & eturns & \\
\hline 49 & $12682 N$ & 4239 & 6613 & 30 Mar & 4522 & 6420.5 & 19 Sep 53 \\
\hline 50 & $12692 \mathrm{~N}$ & 4247 & 6700 & 30 Mar & 4417 & 6618 & 15 Aug 53 \\
\hline
\end{tabular}

*Bottle recovered from bottom 
Released

\begin{tabular}{|c|c|c|c|c|}
\hline Station & Bottie & Lat & Long & Date \\
\hline 50 & $12698 \mathrm{~N}$ & 4241 & 6700 & 30 Mar \\
\hline 51 & & 4246 & 6800 & 30 Mar \\
\hline 52 & & 4240 & 6900 & $30 \mathrm{Mar}$ \\
\hline 53 & $12726 \mathrm{~N}$ & 4238 & 6956 & 31 Mar \\
\hline 54 & $12737 \mathrm{~B}$ & 4237 & 7024 & 31 Mar \\
\hline 54 & $12740 \mathrm{~N}$ & 4237 & 7024 & 31 Mar \\
\hline 54 & $12742 \mathrm{~N}$ & 4237 & 7024 & 31 Mar \\
\hline 54 & $12743 \mathrm{~B}$ & 4237 & 7024 & 31 Mar \\
\hline 54 & $12745 B$ & 4237 & 7024 & 31 Mar \\
\hline 54 & $12746 \mathrm{~N}$ & 4237 & 7024 & 31 Mar \\
\hline 54 & $12748 \mathrm{~N}$ & 4237 & 7024 & 31 Mar \\
\hline 55 & & 4200 & 6953 & 31 Mar \\
\hline 66 & & 4200.5 & 6900.5 & 31 Mar \\
\hline 57 & & 4200 & 6800 & 31 Mar \\
\hline 58 & & 4202 & 6706 & 31 Mar \\
\hline 59 & & 4159 & 6600 & 1 Apr \\
\hline 60 & & 4730 & 6549 & 1 Apr \\
\hline 61 & & 4129 & 6622 & $I \mathrm{Apr}$ \\
\hline 62 & & 4125.5 & 6781 & $1 \mathrm{Apr}$ \\
\hline 63 & $12852 \mathrm{~N}$ & 4103 & 6758 & $1 \mathrm{Apr}$ \\
\hline 64 & $12862 \mathrm{~N}$ & 4050 & 6900 & $1 \mathrm{Apr}$ \\
\hline 65 & $12872 \mathrm{~N}$ & 4036 & 6941 & 2 Apr \\
\hline 66 & $12888 \mathrm{~N}$ & 4055 & 7016 & $2 \mathrm{Apr}$ \\
\hline 66 & $12892 \mathrm{~N}$ & 4055 & 7016 & 2 Apr \\
\hline 67 & $12898 \mathrm{~N}$ & 4712 & 7042 & $2 \mathrm{Apr}$ \\
\hline 67 & $12902 \mathrm{~N}$ & 4112 & 7042 & $2 \mathrm{Apr}$ \\
\hline 67 & $12904 \mathrm{~N}$ & 4112 & 7042 & $2 \mathrm{Apr}$ \\
\hline
\end{tabular}

Recovered

Lat Iong Date

$4505 \quad 6628 \quad 9$ JuI 53 No returns No returns

$4758.37000 \quad 11$ Apr 53

4159.57004 .52 Mar 55

$4154.76958 .1 \quad 11$ Apr 53

$4154.7 \quad 6958.1 \quad 11$ Apr 53

$4203.67010 \quad 22$ Apr 53

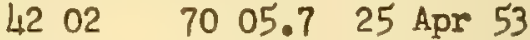

$4157.5 \quad 6959.5 \quad 11$ Apr 53

$4154.7 \quad 6958.1$ 11 Apr 53 No returns

No returns

No returns

No returns

No returns

No returns

No returns

No returns

$4335 \quad 6000 \quad 21$ JuI 53

$\begin{array}{llll}36 & 03.3 \quad 7541 \quad 15 & \text { Oct } 53\end{array}$

$\begin{array}{lllll}40 & 57.7 & 72 & 08.6 \quad 8 \text { Aug } 53\end{array}$

$\begin{array}{lllllll}426.1 & 70 & 42.1 & 1 & \mathrm{JuI} & 53\end{array}$

$\begin{array}{lllllll}419 & 70 & 49 & 14 & \mathrm{JuI} & 53\end{array}$

LI $17 \quad 70 \quad 14.5 \quad 10 \mathrm{May} 53$

$4120.7 \quad 7000.5 \quad 3$ May 53

$4119 \quad 7019.1 \quad 28$ Jun 53 


\section{Released}

\begin{tabular}{|c|c|c|c|c|}
\hline Station & Bottle & Lat & Long & Date \\
\hline 1 & $12914 N$ & 4116.8 & 7100 & 24 Apr \\
\hline 1 & $12916 \mathrm{~N}$ & 4116.8 & 7100 & 24 Apr \\
\hline 2 & 12920N & 4101.5 & 7100.5 & $24 \mathrm{Ap}$ \\
\hline 3 & & 4029 & 7059.5 & $24 A p$ \\
\hline 4 & & 3959 & 7058.5 & $25 \mathrm{Ap}$ \\
\hline 5 & & 4000 & 7000 & $25 \mathrm{Ap}$ \\
\hline 6 & & 4000.5 & 6900 & $25 \mathrm{Ap}$ \\
\hline 7 & & 4000 & 6830 & $25 \mathrm{Ap}$ \\
\hline 8 & & 4030 & 6800 & $25 \mathrm{Ap}$ \\
\hline 9 & & 4700 & 6730 & $25 \mathrm{Ap}$ \\
\hline 10 & & 4715 & 6716 & $25 \mathrm{Ap}$ \\
\hline 11 & & 4145 & 6713 & $26 \mathrm{Ap}$ \\
\hline 12 & $13047 \mathrm{~B}$ & 4215.7 & 6715 & $26 \mathrm{Ap}$ \\
\hline 13 & $13050 \mathrm{~N}$ & 4258.5 & 6715.5 & $26 \mathrm{Ap}$ \\
\hline 13 & $13060 \mathrm{~N}$ & 4258.5 & 6715.5 & $26 \mathrm{Ap}$ \\
\hline$\tilde{I}_{4}$ & $13070 \mathrm{~N}$ & 4340 & 6715 & $26 \mathrm{Ap}$ \\
\hline 14 & $13072 \mathrm{~N}$ & 4340 & 6715 & $26 \mathrm{Ap}$ \\
\hline 15 & 130758 & 43.40 & 6800 & 26 Ap \\
\hline 16 & $13092 \mathrm{~N}$ & 4340 & 6900 & $26 \mathrm{Ap}$ \\
\hline 17 & $13098 \mathrm{~N}$ & 4320 & 6940 & $27 \mathrm{Ap}$ \\
\hline 18 & $13110 \mathrm{~N}$ & 4300 & 7033.6 & $27 \mathrm{Ap}$ \\
\hline 18 & $13113 \mathrm{~B}$ & 4300 & 7033.6 & $27 \mathrm{Ap}$ \\
\hline 19 & $13128 \mathrm{~N}$ & 4300.5 & 7000 & $27 A_{p}$ \\
\hline 19 & $13132 \mathrm{~N}$ & 4300.5 & 7000 & $27 \mathrm{Ap}$ \\
\hline 20 & $13134 \mathrm{~N}$ & 4259 & 6900 & $27 \mathrm{Ap}$ \\
\hline 20 & 13山 $2 \mathrm{~N}$ & 4259 & 6900 & $27 \mathrm{Ap}$ \\
\hline 21. & $13152 \mathrm{~N}$ & 4300 & 6800 & $27 \mathrm{Ap}$ \\
\hline 22 & & 4300 & 6700 & $27 \mathrm{Ap}$ \\
\hline 23 & $13172 \mathrm{~N}$ & 4302 & 6600 & $28 \mathrm{Ap}$ \\
\hline 23 & $13179 B$ & 4302 & 6600 & $28 \mathrm{Ap}$ \\
\hline 24 & $13192 \mathrm{~N}$ & 4302 & 6501 & $28 \mathrm{Ap}$ \\
\hline 25 & & 4215 & 6455 & $28 \mathrm{Ap}$ \\
\hline 26 & & 4214 & 6600 & $28 \mathrm{Ap}$ \\
\hline 27 & & 4215 & 6700 & $29 \mathrm{Ap}$ \\
\hline 28 & & 4213 & 6800 & $29 \mathrm{Ap}$ \\
\hline 29 & & 4212 & 6859.5 & $29 \mathrm{Ap}$ \\
\hline 30 & $13254 \mathrm{~N}$ & 4213 & 7000 & $29 \mathrm{Ap}$ \\
\hline 30 & $13256 \mathrm{~N}$ & 4213 & 7000 & 29 Apr \\
\hline
\end{tabular}

Recovered

Iat Iong Date

$4125 \quad 7057 \quad 24$ May 53

$4123 \quad 7043 \quad 30$ Apr 53

$4121.87137 \quad 24 \mathrm{May} 53$

No returns

No returns

No returns

No returns

No returns

No returns

No returns

No returns

No returns

$\begin{array}{llllll}45 & 19.8 & 64 & 41.8 & 13 & \text { Sep } 53\end{array}$

$4506 \quad 6624 \quad 20$ Sep 53

$\begin{array}{lllll}45 & 02.3 \quad 66 & 53.5 \quad 11 & \text { Aug } 53\end{array}$

$\begin{array}{llllll}43 & 51.7 & 68 & 53.1 & 17 & \text { May } 53\end{array}$

$\begin{array}{lllll}4522 & 6420 & 27 & \text { Sep } 53\end{array}$

$4347 \quad 6609 \quad 10$ Apr 54

$\begin{array}{llll}4300.3 & 7036 \quad 2 \text { Jun } 53\end{array}$

$\begin{array}{llll}43 & 03.370 & 42.9 & 25 \\ 4 & \text { May } 53\end{array}$

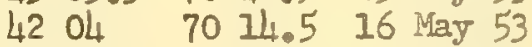

$4121.8 \quad 7137 \quad 10$ Aug 53

$4434.3 \quad 6555 \quad 6$ Sep 53

$5310 \quad 0914 \quad 27 \mathrm{JuI} 54$

$4505 \quad 6625 \quad 4$ Oct 53

$44556519 \quad 3$ Dec 53

$4415.5 \quad 6620.222$ Aug 53 No returns

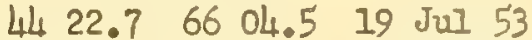

$\begin{array}{lllll}44.5 & 14 & 22.5 & 9 & \text { Sep } 53\end{array}$ $4446.3 \quad 6645 \quad 24$ JuI 53

No returns

No returns

No returns

No returns

No returns

$\begin{array}{lllll}42 & 05 & 70 & 12.5 & 17 \\ 4 a y & 53\end{array}$

$4204.2 \quad 7014.4 \quad 3 \mathrm{May} 53$ 


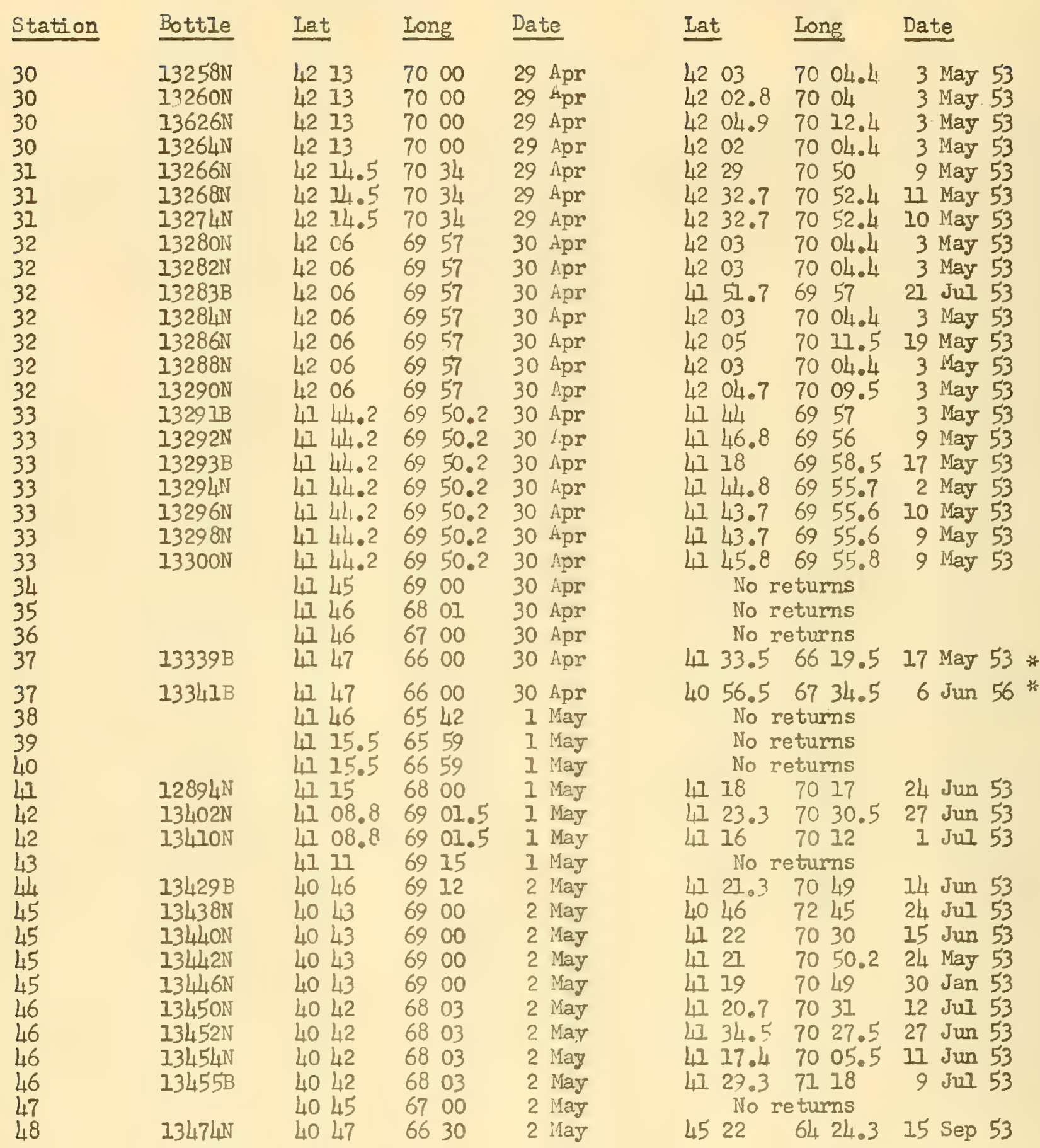

* Bottle recovered from bottom 


\begin{tabular}{|c|c|c|c|c|c|c|c|}
\hline Station & Bottle & Lat & Iong & Date & Iat & Long & Date \\
\hline 49 & & 4730 & 6626.5 & $1 \mathrm{May}$ & \multicolumn{3}{|c|}{ No returns } \\
\hline 50 & $13496 \mathrm{~N}$ & 4215 & 6617.5 & I May & 4506 & 6638.4 & $14 \mathrm{Jul} 53$ \\
\hline 50 & $13497 \mathrm{~B}$ & 4215 & 6617.5 & $1 \mathrm{May}$ & 4426.5 & 6600.5 & 9 Jan 53 \\
\hline 50 & $13500 \mathrm{~N}$ & 4215 & 6617.5 & I May & 4426 & 6610.5 & 16 Oct 53 \\
\hline 50 & $13503 \mathrm{~B}$ & 4215 & 6617.5 & 1 May & 4447.5 & 6547 & $16 \mathrm{Feb} 54$ \\
\hline 51 & $13510 \mathrm{~N}$ & 4319 & 6601 & 1 May & 5640 & 0628 & 5 oct 54 \\
\hline 51 & $13514 \mathrm{~N}$ & 4319 & 6601 & 1 May & 4422 & 6625 & 15 Aug 54 \\
\hline 51 & $13516 \mathrm{~N}$ & 4319 & 6601 & $1 \mathrm{May}$ & 4507 & 6629 & 25 JuI 53 \\
\hline 52 & $13530 \mathrm{~N}$ & 4321 & 6700 & 3 May & 4532 & 6447 & 12 Sep 53 \\
\hline 53 & & 4320 & 6757 & $3 \mathrm{May}$ & \multicolumn{2}{|c|}{ No retums } & \\
\hline 54 & $13546 \mathrm{~N}$ & 4318 & 6900 & 3 May & 4144.2 & 7020 & 4 Jun 53 \\
\hline 54 & $13550 \mathrm{~N}$ & 4318 & 6900 & 3 May & 4154 & 7001 & 14 Aug 53 \\
\hline 55 & $13566 \mathrm{~N}$ & 4318 & 7000 & 4 May & 4339 & 6549.3 & $27 \mathrm{Feb} 54$ \\
\hline 56 & $13568 \mathrm{~N}$ & 4317 & 7031 & 4 May & 4252.3 & 7049 & 16 May 53 \\
\hline 56 & $13570 \mathrm{~N}$ & 4317 & 7031 & $4 \mathrm{May}$ & 4306 & 7039.5 & 27 Jun 53 \\
\hline 56 & $13571 B$ & 4317 & 7031 & 4 May & $43 \quad 18.5$ & 7034 & $28 \mathrm{May} 54$ \\
\hline 56 & $13572 B$ & 4317 & 7031 & 4 May & 4307.8 & 7038.5 & $1 \mathrm{Dec} 54$ \\
\hline 56 & $13573 \mathrm{~B}$ & 4317 & 7031 & 4 May & 4231 & 7052 & 20 May 53 \\
\hline 56 & $13576 \mathrm{~N}$ & 4317 & 7031 & 4 May & 4245 & 7048 & $14 \operatorname{Jan} 53$ \\
\hline 56 & $13578 \mathrm{~N}$ & 4317 & 7031 & 4 May & 4250.6 & 7049 & $16 \mathrm{May} 53$ \\
\hline 57 & $13579 \mathrm{~B}$ & 4300 & 7033 & 4 May & 4204 & 7006.5 & 9 Juา 53 \\
\hline 57 & $13581 \mathrm{~B}$ & 4300 & 7033 & 4 May & 4258.3 & 7046 & 30 Oct 55 \\
\hline 57 & $13586 \mathrm{~N}$ & 4300 & 7033 & 4 May & 4253.8 & 7048.8 & 14 May 53 \\
\hline 57 & $13587 \mathrm{~B}$ & 4300 & 7033 & $4 \mathrm{May}$ & 4255 & 7027 & 29 JuI 56 * \\
\hline 57 & $13588 \mathrm{~N}$ & 4300 & 7033 & 4 May & 4143.9 & 7017.3 & 30 May 53 \\
\hline 58 & $13594 \mathrm{~N}$ & 4242 & 7032.5 & 4 May & 4127 & 7040 & 16 Aug 53 \\
\hline 59 & & 4240 & 7000 & 4 May & No & eturns & \\
\hline 60 & & 4240 & 6900 & 4 May & No $r$ & eturns & \\
\hline 61 & $13638 \mathrm{~N}$ & 4241.5 & 6800 & 4 Мау & 4509 & 6614 & 7 Dec 53 \\
\hline 62 & & 4241 & 6703 & 5 May & No 1 & oturns & \\
\hline 63 & $13654 \mathrm{~N}$ & 4242.5 & 6557 & 5 May & $4423 . ?$ & 6612 & $10 \operatorname{Jan} 53$ \\
\hline 63 & $13656 \mathrm{~N}$ & 4242.5 & 6557 & 5 May & 4502.5 & 6653.5 & 22 Sep 53 \\
\hline 63 & $13658 \mathrm{~N}$ & 4242.5 & 6557 & 5 May & 4420 & 6607.7 & $6 \mathrm{Jul} 53$ \\
\hline 63 & $13662 \mathrm{~N}$ & 4242.5 & 6557 & 5 May & 4433.8 & 6550.8 & 2 JuI 53 \\
\hline 64 & $13668 \mathrm{~N}$ & 4230 & 6500 & 5 May & 4522 & 6423.5 & 8 Sep 53 \\
\hline 64 & $13674 \mathrm{~N}$ & 4230 & 6500 & 5 May & 4520 & 6447 & 20 Sep 53 \\
\hline 65 & $13680 \mathrm{~N}$ & 4200 & 6500 & 5 May & 4520 & 6447 & 4 Aug 53 \\
\hline 66 & & 4200 & 6559.5 & 5 May & No & eturns & \\
\hline 67 & & 4200 & 6700 & 5 May & No : & eturns & \\
\hline 68 & & 4201 & 6800 & 6 May & No: & eturns & \\
\hline 69 & & 4158 & 6900 & $6 \mathrm{May}$ & No : & eturns & \\
\hline 70 & & 4754.5 & 6942 & 6 May & No: & eturns & \\
\hline 71 & & 4131 & 6929 & 6 May & No & eturns & \\
\hline 72 & & 4133.5 & 6902 & 6 May & No & eturns & \\
\hline 73 & & 4125.5 & 6800 & 6 May & No: & eturns & \\
\hline
\end{tabular}

* Bottle recovered from bottom 
Released

\begin{tabular}{|c|c|c|c|c|}
\hline Station & Bottle & Lat & Ions & Date \\
\hline 74 & $13783 \mathrm{~B}$ & 4128.5 & 6700 & 7 May \\
\hline 75 & & 4130 & 6600 & 7 Мау \\
\hline 76 & $13807 \mathrm{~B}$ & 4104 & 6700 & 7 May \\
\hline 76 & $13809 \mathrm{~B}$ & 4104 & 6700 & 7 May \\
\hline 77 & $13827 \mathrm{~B}$ & 4046.5 & 6745 & 7 May \\
\hline 78 & $13842 \mathrm{~N}$ & 4031 & 6813 & 7 May \\
\hline 79 & $13844 \mathrm{~N}$ & 4028 & 6900 & 8 May \\
\hline 79 & $13845 \mathrm{~B}$ & 4028 & 6900 & 8 May \\
\hline 79 & $13846 \mathrm{~N}$ & 4028 & 6900 & 8 May \\
\hline 79 & $13848 \mathrm{~N}$ & 4028 & 6900 & 8 May \\
\hline 79 & $13852 \mathrm{~N}$ & 4028 & 6900 & 8 May \\
\hline 79 & $13854 \mathrm{~N}$ & 4028 & 6900 & 8 May \\
\hline 80 & $13856 \mathrm{~N}$ & 4024 & 7000 & $8 \mathrm{May}$ \\
\hline 81 & $13868 \mathrm{~N}$ & 4710 & 7020 & 8 May \\
\hline 81 & $13872 \mathrm{~N}$ & 4110 & 7020 & 8 May \\
\hline 81 & $13874 \mathrm{~N}$ & 4710 & 7020 & 8 May \\
\hline 81 & $13876 \mathrm{~N}$ & 4110 & 7020 & $8 \mathrm{May}$ \\
\hline
\end{tabular}

Recovered

Iat Iong Date

$4047 \quad 6758 \quad 9$ Aug 54 No returns

$4031 \quad 6847 \quad 28 \mathrm{Jan} 54$

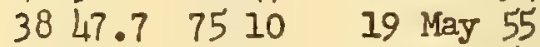

$40 \quad 46.6 \quad 6806 \quad 29 \mathrm{May} 53$

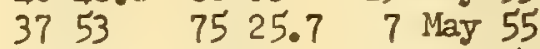

$4739.37007 \quad 29$ Jun 53

$47 \begin{array}{llll}38 & 70 & 14 & \mathrm{JuI} \\ 53\end{array}$

$\begin{array}{lllllll}41 & 27.7 & 71 & 21.8 & 18 & \text { Jun } 53\end{array}$

41 $31 \quad 70 \quad 39.5 \quad 28$ Jun 53

$47 \begin{array}{llll}14.5 & 7006 \quad 13 \mathrm{Jun} 53\end{array}$

$4131.5 \quad 70 \quad 40.3 \quad 31 \mathrm{Aug} 54$

$\begin{array}{lllll}40 & 49 & 72 & 33.7 & 14 \\ 4 & \text { Aug } 53\end{array}$

$4120.8 \quad 70 \quad 39 \quad 10 \mathrm{May} 53$

$4720.8 \quad 70 \quad 39 \quad 16$ May 53

$4720.7 \quad 7040 \quad 9$ Jun 53

$4121 \quad 7046 \quad 13$ Jun 53 
Released

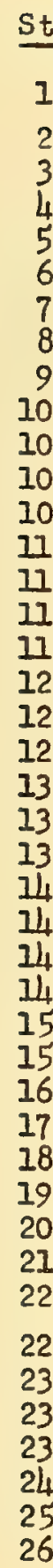

$14150 \mathrm{~N}$
Recovered

Lat Long Date

$4115.77012 .11 \mathrm{Jul} 55$ * No returns

No returns

No returns

No returns

No retums

No returns

No returns

No returns

$4403 \quad 6610$

$4519.5 \quad 6448$

4413.26609

$4520 \quad 6447$

$4520 \quad 6454$

14 Jun 53

$\begin{array}{llll}43 & 01.5 & 66.21 & 27 \\ \text { May }\end{array}$

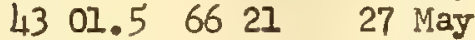

$\begin{array}{llll}43 & 01.5 & 66 & 21 \\ 4 & 27 & \mathrm{May}\end{array}$

$\begin{array}{lllll}43 & 01.5 & 66 & 21 & 27 \\ 4 & \text { May }\end{array}$

$4327 \quad 6615 \quad 27$ May

$4327 \quad 6615 \quad 27$ May

$4327 \quad 6615 \quad 27$ May

$4327 \quad 6615 \quad 27$ May

$\begin{array}{llll}43 & 26.5 & 67 & 00 \quad 27 \\ 4 a y\end{array}$

$\begin{array}{llll}43 & 26.5 & 67 & 00 \quad 27 \\ 4 a y\end{array}$

$\begin{array}{llll}43 & 26.5 & 67 & 00 \quad 27 \\ 4 a y\end{array}$

$4326.5 \quad 6800 \quad 28$ May

$4326.5 \quad 6800 \quad 28 \mathrm{May}$

$4326.56800 \quad 28 \mathrm{May}$

$4328 \quad 6900 \quad 28 \mathrm{May}$

$4328 \quad 6900 \quad 28$ May

$4328 \quad 6900 \quad 28 \mathrm{May}$

$4328 \quad 6900 \quad 28 \mathrm{May}$

$\begin{array}{lll}4327 & 7001 \quad 28 \mathrm{May}\end{array}$

$4327 \quad 7001 \quad 28$ Мау

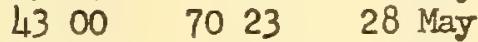

\begin{tabular}{llll}
42 & 35 & 7032 & 28 \\
\hline
\end{tabular}

$4210 \quad 7000 \quad 29$ May

$4210 \quad 6900 \quad 29$ Мay

\begin{tabular}{lllll}
42 & 08 & 68 & 00 & 29 \\
\hline
\end{tabular}

$4210 \quad 6700 \quad 29$ May

$14 \mathrm{I}_{1 \mathrm{~B}} \quad 4208 \quad 6610 \quad 29 \mathrm{May}$

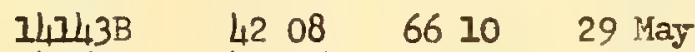

42356610

29 Мay

$4235 \quad 6610$

29 May

$4235 \quad 6610$

29 May

$\begin{array}{llll}42 & 35 & 6500 \quad 30 \mathrm{May}\end{array}$

$4203.5 \quad 6529 \quad 30$ May

$41456545.5 \quad 30$ May
4426

4500

4520

448

4520

4422.7

4457

4450

4504.5

4.41

4422

$\begin{array}{ll}42 & 03\end{array}$

4717

4202.5

4254.5

4420

\section{No returns}

No returns

No returns

No returns

No returns

$4152 \quad 6610$

$4052 \quad 6724.5 \quad 4 \mathrm{JuI} 56$ *

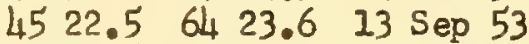

$4519.5 \quad 6448 \quad 25$ JuI 53

$4522.5 \quad 6425$

$4357 \quad 6000$

No returns

No returns
4 Sep 53

13 Sep 53

* Bottle recovered from bottom 
Released

\begin{tabular}{|c|c|c|c|c|}
\hline Station & Bottle & Lat & Long & Date \\
\hline $\begin{array}{l}27 \\
28 \\
29 \\
30\end{array}$ & $14233 B$ & $\begin{array}{ll}47 & 44 \\
47 & 46.5 \\
47 & 45 \\
47 & 41.5\end{array}$ & $\begin{array}{ll}67 & 00 \\
68 & 07 \\
69 & 00 \\
69 & 48.5\end{array}$ & $\begin{array}{l}30 \text { May } \\
30 \text { May } \\
31 \text { May } \\
31 \text { May }\end{array}$ \\
\hline $\begin{array}{l}31 \\
31 \\
32 \\
33\end{array}$ & $\begin{array}{l}14255 \mathrm{~B} \\
14247 \mathrm{~B} \\
14277 \mathrm{~B}\end{array}$ & $\begin{array}{ll}41 & 21 \\
41 & 21 \\
41 & 25 \\
41 & 21\end{array}$ & $\begin{array}{ll}69 & 28 \\
69 & 28 \\
69 & 03 \\
67 & 59\end{array}$ & $\begin{array}{l}31 \text { May } \\
31 \text { May } \\
31 \text { May } \\
31 \text { May }\end{array}$ \\
\hline $\begin{array}{l}34 \\
35 \\
36 \\
37 \\
38 \\
39 \\
40\end{array}$ & & $\begin{array}{ll}41 & 18 \\
41 & 20 \\
41 & 00 \\
40 & 29 \\
40 & 29 \\
40 & 28 \\
40 & 26.5\end{array}$ & $\begin{array}{ll}67 & 01.5 \\
66 & 11 \\
66 & 31 \\
67 & 02 \\
68 & 02 \\
68 & 58 \\
70 & 00\end{array}$ & $\begin{array}{rr}31 & \text { May } \\
1 & \text { Jun } \\
1 & \text { Jun } \\
1 & \text { Jun } \\
1 & \text { Jun } \\
1 & \text { Jun } \\
2 & \text { Jun }\end{array}$ \\
\hline 41 & & 4024.5 & 7057 & 2 Jun \\
\hline $\begin{array}{l}42 \\
43\end{array}$ & $14379 \mathrm{~B}$ & $\begin{array}{ll}40 & 26.5 \\
40 & 31\end{array}$ & $\begin{array}{ll}72 & 00 \\
72 & 58.5\end{array}$ & $\begin{array}{ll}2 & \text { Jun } \\
2 & \text { Jun }\end{array}$ \\
\hline 44 & $14498 \mathrm{~N}$ & 4047 & 7209 & 2 Jun \\
\hline 44 & $14508 \mathrm{~N}$ & $40 \quad 47$ & 7209 & 2 Jun \\
\hline 45 & $14399 B$ & 4059.5 & 7127.5 & 2 Jun \\
\hline 46 & $14404 N$ & 4100 & 7050.5 & 3 Jun \\
\hline 46 & $14408 \mathrm{~N}$ & 4100 & 7050.5 & 3 Jun \\
\hline 46 & $14472 \mathrm{~N}$ & 4700 & 7050.5 & 3 Jun \\
\hline
\end{tabular}

Recovered

Iat Long Date

No returns

No returns

No returns

LO $58 \quad 70 \mathrm{L4} \quad 16$ Oct 56 *

$3959.56919 \quad 4$ Oct 56 *

404670351 Nor $55 *$

No returns

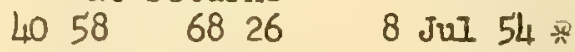

No returns

No returns

No returns

No returns

No returns

No returns

No returns

No returns

$\begin{array}{llll}38 & 43 & 74 & 55\end{array} 24$ Sep 53 No returns

$\begin{array}{llllll}40 & 37.3 \quad 73 & 18.4 & 18 & \text { Jun } & 53\end{array}$

$4104 \quad 7151.6 \quad 10 \mathrm{Jun} 53$

$4107 \quad 7130 \quad 31 \mathrm{Mar} 54$

$\begin{array}{llll}4124 & 7027 & 5 \text { JuI } 53\end{array}$

$4119.5 \quad 704999$ JuI 53

$4123.8 \quad 70 \quad 30.3 \quad 14$ Jun 53

* Bottle recovered from bottom 
Released

\begin{tabular}{|c|c|c|c|c|}
\hline Station & Bottle & Lat & Long & Date \\
\hline 1 & 161118 & 4117.5 & 7100 & $21 \mathrm{Feb}$ \\
\hline 2 & & 4100 & 7101 & $21 \mathrm{Feb}$ \\
\hline 3 & & 4030 & 7100 & $21 \mathrm{Feb}$ \\
\hline 4 & & 4000 & 7100 & $21 \mathrm{Feb}$ \\
\hline 5 & & 3958.5 & 7003 & $22 \mathrm{Feb}$ \\
\hline 6 & $16166 \mathrm{~N}$ & 4000 & 6902 & $22 \mathrm{Feb}$ \\
\hline 7 & & 4000 & 6800 & $22 \mathrm{Feb}$ \\
\hline 8 & $16185 \mathrm{~B}$ & 4036 & 6743 & $22 \mathrm{Feb}$ \\
\hline 9 & & 4100 & 6731 & $22 \mathrm{Feb}$ \\
\hline 10 & $16220 \mathrm{~N}$ & LI. 30 & $\begin{array}{lll}67 & 08.5\end{array}$ & $23 \mathrm{Feb}$ \\
\hline 11 & & $\begin{array}{ll}42 & 02.5\end{array}$ & 6650 & $23 \mathrm{Feb}$ \\
\hline 12 & $16238 \mathrm{~N}$ & 4228 & 6629 & $23 \mathrm{Feb}$ \\
\hline 12 & $16239 \mathrm{~B}$ & 4228 & 6629 & 23 Feb \\
\hline 13 & & 4300 & 6616 & $23 \mathrm{Feb}$ \\
\hline 14 & & 4318 & 6603.5 & $23 \mathrm{Feb}$ \\
\hline 15 & & $4 \longdiv { 4 0 0 }$ & 6630 & $23 \mathrm{Feb}$ \\
\hline 16 & $16289 B$ & 4355 & 6703 & 23 Feb \\
\hline 17 & $16304 \mathrm{~N}$ & 4347 & 6800 & $24 \mathrm{Feb}$ \\
\hline 18 & & 4344 & 6900 & $24 \mathrm{Feb}$ \\
\hline 19 & $16320 \mathrm{~N}$ & 4339 & 6942 & $24 \mathrm{Feb}$ \\
\hline 19 & $16323 B$ & 4339 & 6942 & 24 Feb \\
\hline 19 & $1632 \mathrm{LN}$ & 4339 & 6942 & $24 \mathrm{Feb}$ \\
\hline 20 & $16331 \mathrm{~B}$ & 4300 & 7025 & $24 \mathrm{Feb}$ \\
\hline 20 & $1 6 3 \longdiv { 4 0 N }$ & 4300 & 7025 & $24 \mathrm{Feb}$ \\
\hline $2 I$ & $16344 \mathrm{~N}$ & 4234.2 & 7025 & $24 \mathrm{Feb}$ \\
\hline 22 & $16362 \mathrm{~N}$ & 4200 & 7030 & $25 \mathrm{Feb}$ \\
\hline 22 & $16364 \mathrm{~N}$ & 4200 & 7030 & $25 \mathrm{Feb}$ \\
\hline 23 & & 4221 & 6958 & $25 \mathrm{Feb}$ \\
\hline 24 & $16382 \mathrm{~N}$ & 4239.5 & 6959 & $25 \mathrm{Feb}$ \\
\hline 25 & 163898 & 4300 & 6956 & $25 \mathrm{Feb}$ \\
\hline 25 & $16395 \mathrm{~B}$ & 4300 & 6956 & $25 \mathrm{Feb}$ \\
\hline 26 & $16409 \mathrm{~B}$ & 4257 & 6859 & $25 \mathrm{Feb}$ \\
\hline 27 & $16475 \mathrm{~B}$ & 4245 & 6801 & $25 \mathrm{Feb}$ \\
\hline 28 & $16429 \mathrm{~B}$ & 4243 & 6700 & $25 \mathrm{Feb}$ \\
\hline 29 & $16448 \mathrm{~N}$ & 4243 & 6600 & $26 \mathrm{Feb}$ \\
\hline 30 & & 4247 & 6504 & $26 \mathrm{Feb}$ \\
\hline 31 & $16462 \mathrm{~N}$ & 4220 & 6530 & $26 \mathrm{Feb}$ \\
\hline 32 & & 4201 & 6554 & $26 \mathrm{Feb}$ \\
\hline 33 & $16488 \mathrm{~N}$ & 4758 & 6701 & 26 Feb \\
\hline 34 & $16497 \mathrm{~B}$ & 4204 & 6803 & $26 \mathrm{Feb}$ \\
\hline 34 & $16503 \mathrm{~B}$ & 4204 & 6803 & $26 \mathrm{Feb}$ \\
\hline 34 & $16507 \mathrm{~B}$ & 4204 & 6803 & $26 \mathrm{Feb}$ \\
\hline 35 & $16510 \mathrm{~N}$ & 4200 & 6900 & $27 \mathrm{Feb}$ \\
\hline 35 & $16516 \mathrm{~N}$ & 4200 & 6900 & $27 \mathrm{Feb}$ \\
\hline
\end{tabular}

Recovered

Lat Long Date

$4120.6 \quad 70 \quad 47.1 \quad 30$ Mar 55

No returns

No returns

No returns

No returns

$3221.5 \quad 6440 \quad 4$ Dec 55 No returns

$5245935 \quad 29$ Aug 56 No returns

$\begin{array}{llllll}40 & 01 & 74 & 03.3 & 15 & \text { oct } 55\end{array}$ No returns

$4750 \quad 6956.6 \quad 14$ Jun 55 $41496956.5 \quad 15$ oct 55 No returns No returns No returns

$4422.266 \quad 05.2 \quad 23$ Aug 55 $4130 \quad 7102.6 \quad 23$ JuI 55 No returns

4344.56959 .425 Aug 55 $\begin{array}{llllll}42 & 02 & 70 & 05.7 & 22 & \text { May } 55\end{array}$ $4719.4 \quad 7045.4 \quad 4$ Jun 55 $4720.8 \quad 70 \quad 30 \quad 10 \mathrm{Jul} 55$ $4046.5 \quad 72 \quad 42.6 \quad 14$ Aug 55 $4053 \quad 72 \quad 21 \quad 15$ Aug 55 $\begin{array}{lllll}37 & 11.4 & 00 & 11 & \text { Oct } 55\end{array}$ $4047.3 \quad 72 \quad 40 \quad 17$ Aug 55 No returns

$4220.7 \quad 7040 \quad 20$ Aug 55 $3602 \quad 7540 \quad 26$ Sep 55 $4148 \quad 6956.3 \quad 10$ May 55 $\begin{array}{llllll}42 & 02 & 70 & 10.1 & 7 & \text { May } 55\end{array}$ LI 237002.25 Aug 55 $\begin{array}{llllll}42 & 18 & 70 & 53.8 & 20 & \mathrm{JuI} \\ 55\end{array}$ $43 \quad 47.5 \quad 6519.2 \quad 23$ Jun 55 No retums

44 $13 \quad 6846 \quad 21$ Aug 56 No returns

$\begin{array}{lllll}39 & 26.5 & 74 & 20 & 18 \text { Sep } 55\end{array}$ $4234.5 \quad 7045 \quad 13$ Jun 55 $4249 \quad 7049 \quad 13$ Nov 55 $\begin{array}{llll}4505 & 6628 & 9 & 0 \text { ct } 55\end{array}$ $\begin{array}{lllll}41 & 27.8 & 71 & 21.7 \quad 1 \text { Aug } 55\end{array}$ $\begin{array}{lllll}40 & 28.3 & 74 & 00 & 14 \\ \text { JuI } 55\end{array}$ 
Released

\begin{tabular}{|c|c|c|c|c|}
\hline Station & Bottle & Lat & Long & Date \\
\hline 36 & & 4200 & 6956 & $27 \mathrm{Feb}$ \\
\hline 37 & & 4130 & 6942 & $27 \mathrm{Feb}$ \\
\hline 38 & & 4125 & 6855 & $27 \mathrm{Feb}$ \\
\hline 39 & & 4726 & 6802 & $27 \mathrm{Feb}$ \\
\hline 40 & $16578 \mathrm{~N}$ & 4730 & 6705 & $28 \mathrm{Feb}$ \\
\hline 47 & & 4129 & 6600 & $28 \mathrm{Feb}$ \\
\hline 42 & $16602 \mathrm{~N}$ & 4114 & 6606.5 & $28 \mathrm{Feb}$ \\
\hline 43 & & 4101 & 6624 & $28 \mathrm{Feb}$ \\
\hline 44 & & 4101 & 6700 & $28 \mathrm{Feb}$ \\
\hline 45 & & 4100 & 6758 & $28 \mathrm{Feb}$ \\
\hline 46 & & 4100 & 6917 & 1 Mar \\
\hline 47 & $1665 \mathrm{LN}$ & 4030 & 6857 & 1 Mar \\
\hline 48 & & 4028 & 6800 & 1 Mar \\
\hline 49 & & 4032 & 6658.5 & 1 Mar \\
\hline 50 & & 4108 & 6631 & 1 Mar \\
\hline 51 & & 4130 & 6620 & 2 Mar \\
\hline 52 & & 4200 & 6608 & 2 Mar \\
\hline 53 & $16726 \mathrm{~N}$ & 4222 & 6600 & 2 Mar \\
\hline 54 & & 4309.5 & 6527 & 2 Mar \\
\hline
\end{tabular}

Recovered

Lat Long Date

No returns

No returns

No returns

No returns

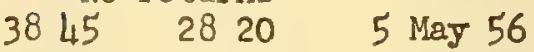

No returns

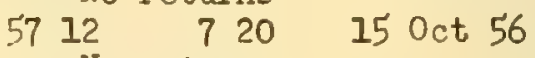

No returns

No returns

No returns

No returns

$5234956 \quad 5$ JuI 56

No returns

No $r$ eturns

No returns

No returns

No returns

$4354.86446 \quad 21$ Aug 55 
Released

\begin{tabular}{|c|c|c|c|c|}
\hline Station & Bottle & Iat & Long & Date \\
\hline 1 & & 4117.5 & 7100 & 19 Mar \\
\hline 2 & & 4100 & 7100 & 19 Mar \\
\hline 3 & & 4028.8 & 7100.8 & 19 Mar \\
\hline 4 & & 4001.5 & 7100 & 19 Mar \\
\hline 5 & & 3959.5 & 6950 & 19 Mar \\
\hline 6 & & 4002.4 & 6857 & 20 Mar \\
\hline 7 & & $40 \quad 04.2$ & 6758.5 & $20 \mathrm{Mar}$ \\
\hline 8 & & $40 \quad 30.4$ & 6744.5 & 20 Mar \\
\hline 9 & $16854 \mathrm{~N}$ & 4101 & 6732.2 & 20 Mar \\
\hline 10 & $16863 \mathrm{~B}$ & 4139.6 & 6710.2 & $20 \mathrm{Mar}$ \\
\hline 10 & 16867B & 4139.6 & 6710.2 & 20 Mar \\
\hline 17 & & 4200 & 6657.2 & 20 Mar \\
\hline 12 & $16882 \mathrm{~N}$ & 4233.6 & 6634 & 21 Mar \\
\hline 12 & $16884 \mathrm{~N}$ & 4233.6 & 6634 & 21 Mar \\
\hline 12 & $16889 \mathrm{~B}$ & 4233.6 & 6634 & $21 \mathrm{Mar}$ \\
\hline 13 & I6897B & 4300 & 6617.8 & 21 Mar \\
\hline 14 & $16909 \mathrm{~B}$ & 4320 & 6606.8 & $21 \mathrm{Mar}$ \\
\hline 15 & $16951 \mathrm{~B}$ & 4343 & 6618.2 & 21 Mar \\
\hline 16 & $16957 \mathrm{~B}$ & 4400 & 6630 & 21 Mar \\
\hline 16 & $16958 \mathrm{~N}$ & 4400 & 6630 & 21 Mar \\
\hline 17 & $16922 \mathrm{~N}$ & 4355.5 & 6701 & 21 Mar \\
\hline 17 & $16926 \mathrm{~N}$ & 4355.5 & 6701 & $21 \mathrm{Mar}$ \\
\hline 18 & & 4352 & 6804 & $21 \mathrm{Mar}$ \\
\hline 19 & $16970 \mathrm{~N}$ & 4341.5 & 6900 & 22 Mar \\
\hline 19 & $16973 \mathrm{~B}$ & $43 \quad 47.5$ & 6900 & 22 Mar \\
\hline 19 & $16976 \mathrm{~N}$ & 4347.5 & 6900 & 22 Mar \\
\hline 20 & $16978 \mathrm{~N}$ & 4335 & 6943 & 22 Mar \\
\hline 20 & $16984 \mathrm{~N}$ & 4335 & 6943 & 22 Mar \\
\hline 21 & $16992 \mathrm{~N}$ & 4321 & 7006.5 & 22 Mar \\
\hline 21 & $16993 \mathrm{~B}$ & 4321 & 7006.5 & 22 Mar \\
\hline 21 & $16996 \mathrm{~N}$ & 4321 & 7006.5 & 22 Mar \\
\hline 21 & $16998 \mathrm{~N}$ & 4321 & 7006.5 & 22 Mar \\
\hline 22 & $17002 \mathrm{~N}$ & 4300 & 7031 & $22 \mathrm{Mar}$ \\
\hline 22 & $17006 \mathrm{~N}$ & 4300 & 7031 & 22 Mar \\
\hline 22 & $17010 \mathrm{~N}$ & 4300 & 7031 & 22 Mar \\
\hline 22 & $17011 B$ & 4300 & 7031 & $22 \mathrm{Mar}$ \\
\hline 22 & $17012 \mathrm{~N}$ & 4300 & 7031 & 22 Mar \\
\hline 23 & $17015 \mathrm{~B}$ & 4256.8 & 7001 & 23 Mar \\
\hline 23 & $17016 \mathrm{~N}$ & 4256.8 & 7001 & $23 \mathrm{Mar}$ \\
\hline 23 & $17017 \mathrm{~B}$ & 4256.8 & 7001 & $23 \mathrm{Mar}$ \\
\hline 23 & $17020 \mathrm{~N}$ & 4256.8 & 7001 & 23 Mar \\
\hline 23 & $1702 \mathrm{LN}$ & 4256.8 & 7001 & 23 Mar \\
\hline 24 & $17025 \mathrm{~B}$ & 4255 & 6855.7 & 23 Mar \\
\hline 24 & $17027 \mathrm{~B}$ & 4255 & 6855.7 & 23 Mar \\
\hline
\end{tabular}

Recovered

Lat Long Date

No returns

No r eturns

No returns

No returns

No returns

No returns

No returns

No returns

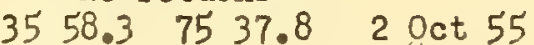

$5710 \quad 0722 \quad 10$ ct 56

$4052 \quad 7224 \quad 14$ Aug 55 No returns

$4504 \quad 6634 \quad 26$ oct 55

4. $24 \quad 6612 \quad 10 \mathrm{JuI} 55$

$4434.3 \quad 6555 \quad 5$ JuI 55

$\begin{array}{lllll}45 & 06.4 & 64 & 56.5 & 21 \\ \text { Jun } 55\end{array}$

$4430 \quad 6606 \quad 27$ Apr 55

$4500 \quad 6657 \quad 24 \mathrm{Jul} 55$

$4433 \quad 6602 \quad 29$ Mar 55

$4509 \quad 6450 \quad 12$ Jun 55

$4444.266 \quad 45.5 \quad 27$ Apr 55

$4320.67029 .5 \quad 11$ May 55 No returns

$4225.7 \quad 7056.1 \quad 10$ May 55

$4239.470 \quad 42.2 \quad 21$ May 55

$4203.1 \quad 7038.6 \quad 29$ Apr 55

$4130.57104 \quad 6 \mathrm{JuI} 55$

$4156 \quad 7033.7 \quad 10$ Jun 55

$4121 \quad 7047.4 \quad 5$ Jun 55

$4140 \quad 6957.1 \quad 4$ JuI 55

$4727.7 \quad 70 \quad 39.5 \quad 4$ Jun 55

$\begin{array}{llll}4202 \quad 70 & 05.820 \\ 4 a y & 55\end{array}$

$4204.9 \quad 70 \quad 10.6 \quad 29$ Apr 55

$4057.27210 \quad 25$ Jun 55

$4155.6 \quad 70 \quad 32.8 \quad$ I May 55

$\begin{array}{lllll}42 & 03.7 \quad 70 & 09.5 & 25 & \text { May } 55\end{array}$

$\begin{array}{llllll}37 & 29.7 & 75 & 38.8 & 18 & \text { Sep } 55\end{array}$

$\begin{array}{llllll}35 & 21.2 & 75 & 30 & 11 \\ \text { JuI } & 56\end{array}$

$\begin{array}{llll}42 & 06.6 & 70 & 40\end{array} 3$ May 55

$4755.6 \quad 70 \quad 32.8 \quad 30 \mathrm{Apr} 55$

$47 \quad 49.2 \quad 6956.4 \quad 13$ Jun 55

$4144.17020 \quad 25 \operatorname{Mar} 56$

$4156 \quad 70 \quad 33.6 \quad 15$ May 55

$\begin{array}{llll}42 & 03.570 & 08.4 \quad 22 \\ \text { May } 55\end{array}$ 
Released

\begin{tabular}{|c|c|c|c|c|}
\hline Station & Bottle & Iat & Long & Date \\
\hline 24 & I7030N & 4255 & 6855.7 & 23 Mar \\
\hline 24 & $17031 \mathrm{~B}$ & 4255 & $68 \quad 55.7$ & 23 Mar \\
\hline 24 & $17036 \mathrm{~N}$ & 425 & 6855.7 & 23 Var \\
\hline 25 & $17043 \mathrm{~B}$ & 4252.5 & 6800 & 23 Var \\
\hline 26 & $17054 \mathrm{~N}$ & 4249.4 & 6702.2 & $24 \mathrm{Mar}$ \\
\hline 26 & $17058 \mathrm{~N}$ & 4249.4 & 6702.2 & 24 Mar \\
\hline 27 & $17066 \mathrm{~N}$ & 4245 & 6557 & $24 \mathrm{Mar}$ \\
\hline 27 & $17068 \mathrm{~N}$ & 4245 & $6557^{\circ}$ & 24 Iar \\
\hline 27 & $17072 N$ & 4245 & 6557 & 24 Mar \\
\hline 28 & & 4240.5 & 6459.5 & 24 Mar \\
\hline 29 & & 4216 & 6502 & $24 \mathrm{Mar}$ \\
\hline 30 & $\ldots$ & 4221.5 & 6622 & $24 \mathrm{Mar}$ \\
\hline 31 & & 4222 & 6702 & 25 Mar \\
\hline 32 & & 4218 & 6759 & $25 \mathrm{Mar}$ \\
\hline 33 & $17137 \mathrm{~B}$ & 4213.3 & 6900 & $25 \mathrm{Mar}$ \\
\hline 33 & $17139 \mathrm{~B}$ & 4213.3 & 6900 & 25 Mar \\
\hline 34 & & 4212.7 & 7008.2 & 25 Mar \\
\hline 35 & $17165 \mathrm{~B}$ & 4215 & $70 \mathrm{Ln}$ & 25 Mar \\
\hline 36 & $17175 \mathrm{~B}$ & 4207.3 & 6958 & $26 \mathrm{Mar}$ \\
\hline 36 & $17180 \mathrm{~N}$ & 4207.3 & 6958 & $26 \mathrm{Mar}$ \\
\hline 37 & & $4145^{\circ}$ & 6947 & 26 Mar \\
\hline 38 & $17198 \mathrm{~N}$ & 4144.5 & 6852 & $26 \mathrm{Mar}$ \\
\hline 38 & $17200 N$ & 4744.5 & 6852 & $26 \mathrm{Mar}$ \\
\hline 39 & & 4146 & 6802.5 & 26 Mar \\
\hline X兴品 & $17262 \mathrm{~N}$ & $4] 59$ & 6959 & 28 Mar \\
\hline$"$ & $17264 \mathrm{~N}$ & 4159 & 6959 & 28 Mar \\
\hline$"$ & $17266 \mathrm{~N}$ & 4759 & 6959 & 28 Nar \\
\hline$" 1$ & $17272 \mathrm{~N}$ & 4759 & 6959 & 28 Mar \\
\hline$" 1$ & $17276 \mathrm{~N}$ & 4159 & 6959 & $28 \mathrm{Mar}$ \\
\hline$n$ & $27277 \mathrm{~B}$ & 4759 & 6959 & 28 Mar \\
\hline$\pi$ & $17280 \mathrm{~N}$ & 4759 & 6959 & 28 Mar \\
\hline$"$ & $17282 \mathrm{~N}$ & 4159 & 6959 & 28 Mar \\
\hline$n$ & $17283 B$ & 4759 & 6959 & $28 \operatorname{Mar}$ \\
\hline$" 1$ & $17284 \mathrm{~N}$ & 4159 & 6959 & $28 \operatorname{Mar}$ \\
\hline$" 1$ & $17288 \mathrm{~N}$ & 4159 & 6959 & $28 \mathrm{Mar}$ \\
\hline 40 & & 4758 & 6949 & 29 Mar \\
\hline 41 & $17304 \mathrm{~N}$ & 4155.8 & 6900 & 29 Mar \\
\hline 41 & $17307 \mathrm{~B}$ & 4155.9 & 6900 & $29 \mathrm{Mar}$ \\
\hline 42 & $17316 \mathrm{~N}$ & 4759.5 & 6801.8 & $29 \operatorname{Mar}$ \\
\hline 43 & $17336 \mathrm{~N}$ & 4200 & 6701 & $29 \mathrm{Mar}$ \\
\hline 44 & $17344 \mathrm{~N}$ & 4153.5 & 6558.8 & 29 Mar \\
\hline 45 & & 4255.3 & 6532 & 29 Mar \\
\hline 46 & $17370 \mathrm{~N}$ & 4229.6 & 6502.8 & 30 Mar \\
\hline 47 & & 4227.3 & 6600 & 30 Mar \\
\hline
\end{tabular}

Recovered

Lat Long Date

$4055.3 \quad 72 \quad 15 \quad 15$ Aug 55 $4122.5 \quad 70 \quad 30.3 \quad 1$ Jun 55 $\begin{array}{llllll}39 & 47.5 & 74 & 08.4 & 15 & \text { Sep } 55\end{array}$ $\begin{array}{llll}3514 & 7531 & 4 & \text { Oct } 55\end{array}$ $4500.5 \quad 6657 \quad 20$ oct 55 $\begin{array}{llll}44 & 00.5 & 66 & 09.2 \quad 19 \\ \text { May } 55\end{array}$

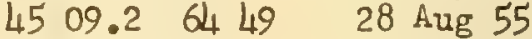
$4340.6 \quad 7010 \quad 27$ Aug 55

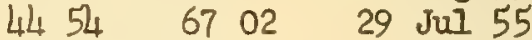

No returns

No returns

No returns

No returns

No returns

$4131 \quad 7104.2 \quad 5$ Jun 55 $4139.4 \quad 6959.129$ May 55 No returns

$\begin{array}{llllll}41 & 17.8 & 70 & 06.3 \quad 3 & \mathrm{JuI} & 55\end{array}$ $4111.57134 \quad 21$ Jun 55 $4138 \quad 7038.6 \quad 4$ Jun 55 No returns

$40 \quad 56.5 \quad 72 \quad 11.7 \quad 13$ Aug 55 $4137.8 \quad 70 \quad 14.5 \quad 27$ JuI 55 No returns

$41 \begin{array}{llll}17.7 & 70 & 06.8 \quad 1 \text { May } 55\end{array}$

$41 \quad 17.5 \quad 70 \quad 05.7 \quad 8$ Apr 55

47 $17.7 \quad 7006.8 \quad 25$ Apr 55

$41 \quad 17.8 \quad 7006.5 \quad 5$ Apr 55

$47 \quad 39.3 \quad 6957.5 \quad 17$ Apr 55

$47 \begin{array}{llll}17.7 & 70 & 08 & 17 \\ \text { Apr } 55\end{array}$

41 $17 \quad 7005$ 19 Anr 55

$\begin{array}{lllllll}37 & 52.8 & 75 & 20.7 & 2 & \text { Oct } 55\end{array}$

$\begin{array}{llllll}39 & 31 & 74 & 18 & 16 & \text { Oct } 55\end{array}$

$4117.7 \quad 7009 \quad 17$ May 55

$47 \begin{array}{llll}17.8 & 70 & 06.3 \quad 30 & \text { Jan } 55\end{array}$ No returns

$4048 \quad 7244 \quad 17$ Aug 55

$4123 \quad 7027 \quad 28$ May 55

3654.976 ol.? 3 oct 55

$4130.3 \quad 7041.5 \quad 6$ Aug 55

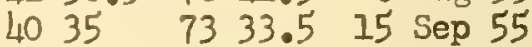
No returns

$4320 \quad 7027 \quad 22$ May 55 No returns 
Released

\begin{tabular}{|c|c|c|c|c|}
\hline Station & BottIe & Lat & Long & Date \\
\hline 48 & & 4226.5 & 6625 & $30 \mathrm{Mar}$ \\
\hline 49 & & 4158 & 6614 & 30 Mar \\
\hline 50 & & 4132.3 & 6600.3 & 30 Mar \\
\hline 51 & $17430 \mathrm{~N}$ & 4127 & 6658.5 & 30 Mar \\
\hline 51 & $17432 \mathrm{~N}$ & 4727 & 6658.5 & 30 Mar \\
\hline 52 & $17443 B$ & 4147 & 6700 & 31 Mar \\
\hline 53 & $17448 \mathrm{~N}$ & 4746.8 & 6600 & 31 Mar \\
\hline 53 & $174501 \mathrm{~N}$ & 4746.8 & 6600 & 31 Mar \\
\hline 54 & & 4144 & 6540.1 & 31 Mar \\
\hline 55 & $17470 \mathrm{~N}$ & 4115.3 & 6606.2 & 31 Mar \\
\hline 6 & $17487 \mathrm{~B}$ & 4110 & 6632 & 31 Mar \\
\hline 57 & & 4103 & 6714 & 31 Mar \\
\hline 58 & & 4052.5 & 6800.2 & 31 Mar \\
\hline 59 & & 4044.5 & 6837.6 & 1 Apr \\
\hline 60 & $17540 N$ & 4036.5 & 6918.5 & $1 \mathrm{Apr}$ \\
\hline 61 & & 4037 & 6953 & $1 \mathrm{Apr}$ \\
\hline 62 & & 4054.2 & 7027 & $1 \mathrm{Apr}$ \\
\hline 63 & $17572 \mathrm{~N}$ & 4109 & 7048.5 & $1 \mathrm{Apr}$ \\
\hline
\end{tabular}

Recovered

Lat Long Date

No returns

No returns

No returns

$\begin{array}{llllll}41 & 18.5 & 70 & 47.5 & 23 & \text { JuI } 55\end{array}$

$4120.8 \quad 7030 \quad 16 \mathrm{Jul} 55$

$3932.6 \quad 74 \quad 15.125$ Aug 55

$4139.4 \quad 6958.2 \quad 6$ Aug 55

$\begin{array}{llllll}41 & 17.4 & 70 & 05.7 & 13 & \mathrm{JuI} \\ 55\end{array}$ No returns

$40 \quad 50.5 \quad 72 \quad 28.7 \quad 11$ Aug 55

$\begin{array}{lllll}35 & 56.9 & 75 & 37 & 22\end{array}$

No returns

No returns

No returns

$\begin{array}{llll}38 & 20 & 75 & 05\end{array}$ 7. Sep 55

No returns

No returns

$\begin{array}{llll}37 & 1 & 7528.1 & 20\end{array}$ 
Released

\begin{tabular}{|c|c|c|c|c|}
\hline Station & Bottle & Lat & Long & Date \\
\hline 1 & & 4119 & 7059 & $19 \mathrm{Apr}$ \\
\hline 2 & $17592 \mathrm{~N}$ & 4100.5 & 7101.5 & 19 Apr \\
\hline 3 & & 41 31.5 & 7100.5 & 19 Apr \\
\hline 4 & & 4008.5 & 7058.5 & $19 \mathrm{Apr}$ \\
\hline 5 & & 3956.5 & 6959 & 20 Apr \\
\hline 6 & & 3954 & 6911 & 20 Apr \\
\hline $6 a$ & & 3953.5 & 6852.5 & $20 \mathrm{Apr}$ \\
\hline 7 & 17667B & 3959.3 & 6801 & 20 Apr \\
\hline 8 & & 4031 & 6747 & $20 \mathrm{Apr}$ \\
\hline 9 & $17686 \mathrm{~N}$ & LI 02.5 & 6729 & $20 \mathrm{Apr}$ \\
\hline 10 & $17704 \mathrm{~s}$ & 4132 & 6708.5 & 20 Apr \\
\hline 11 & $17710 \mathrm{~N}$ & 4202 & 6650 & $21 \mathrm{Apr}$ \\
\hline 11 & $17717 \mathrm{~B}$ & 1202 & 6650 & $27 \mathrm{Apr}$ \\
\hline 12 & & 4216.2 & 6641 & 21 Apr \\
\hline 13 & & 4237.5 & 6623 & 21 Apr \\
\hline I4 & & 4301.5 & 6619 & 21 Apr \\
\hline 15 & & 4319 & 6605 & 21 Apr \\
\hline 16 & & 4341 & 6618.5 & 21 Apr \\
\hline 17 & $17782 \mathrm{~N}$ & W4 00 & 6627.8 & 21 Apr \\
\hline 17 & $17784 \mathrm{~N}$ & 4400 & 6627.8 & 21 Apr \\
\hline 18 & $17797 \mathrm{~B}$ & 4357.5 & 6703 & 21 Apr \\
\hline 19 & $17812 \mathrm{~N}$ & 4355.5 & 6730 & $21 \mathrm{Apr}$ \\
\hline 20 & $17826 \mathrm{~N}$ & 4350 & 6757.2 & $22 \mathrm{Apr}$ \\
\hline 20 & $17828 \mathrm{~N}$ & 4350 & 6757.2 & $22 \mathrm{Apr}$ \\
\hline 21 & $17832 \mathrm{~N}$ & $43 \quad 41.5$ & 6823.5 & $22 \mathrm{Apr}$ \\
\hline 21 & $17835 \mathrm{~B}$ & 4341.5 & 6823.5 & 22 Apr \\
\hline 22 & $17843 \mathrm{~B}$ & 4336.1 & 6855 & 22 Apr \\
\hline 22 & $1784 \mathrm{LN}$ & 4336.1 & 6855 & 22 Apr \\
\hline 22 & $17847 \mathrm{~B}$ & 4336.1 & 6855 & 22 Apr \\
\hline 22 & $17850 \mathrm{~N}$ & 4336.1 & 6855 & $22 \mathrm{Apr}$ \\
\hline 23 & $17854 \mathrm{~N}$ & 4329.5 & 6933.5 & 22 Apr \\
\hline 23 & 178558 & 4329.5 & 6933.5 & 22 Apr \\
\hline 23 & $17856 \mathrm{~N}$ & 4329.5 & 6933.5 & $22 \mathrm{Apr}$ \\
\hline 23 & $17860 \mathrm{~N}$ & 4329.5 & 6933.5 & $22 \mathrm{Apr}$ \\
\hline 23 & $17864 \mathrm{~N}$ & 4329.5 & 6933.5 & $22 \mathrm{Apr}$ \\
\hline 24 & $17866 \mathrm{~N}$ & 4320 & 7001 & $22 \mathrm{Apr}$ \\
\hline 24 & $17868 \mathrm{~N}$ & 4320 & 7001 & $22 \mathrm{Apr}$ \\
\hline 24 & $17871 B$ & $\angle 320$ & 7001 & 22 Apr \\
\hline 24 & $178^{\circ}$ & 1320 & 7001 & $22 \mathrm{Apr}$ \\
\hline 25 & $17878 \mathrm{~N}$ & 4301.5 & 7021 & 22 Apr \\
\hline 25 & $17883 \mathrm{~B}$ & 4301.5 & 7021 & 22 Apr \\
\hline 25 & $17884 \mathrm{~N}$ & 4301.5 & 7021 & 22 Apr \\
\hline
\end{tabular}

Recovered

Lat Long Date No returns

$3753.57525 \quad 24$ Aug 55 No returns

No returns

No returns

No returns

No returns

$5900 \quad 0300 \quad 2$ Nov 56

No returns

$4120.8 \quad 7033.1 \quad 8 \quad \mathrm{Jul} 55$

$\begin{array}{llll}38 & 25 & 31 & 10\end{array} 12$ May 56

$4034.7 \quad 7350 \quad 13$ Aug 55

$\begin{array}{llll}40 & 39 & 73 & 07.6 \quad 3 \text { Sep } 55\end{array}$

No returns

No returns

No returns

No returns

No returns

$4202.6 \quad 7013 \quad 25$ Jun 55

$\begin{array}{llll}4430 & 66 & 06 \quad 8 \text { Sep } 55\end{array}$

$\begin{array}{llllllllllll}43 & 35.8 & 70 & 13.1 & 8 \text { May } 55\end{array}$

$4344.7 \quad 7002 \quad 10$ Sep $55^{\circ}$

$4740 \quad 6956 \quad 17$ Oct 55

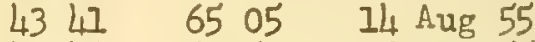

$4748 \quad 6957 \quad 6$ Nor 55

$\begin{array}{lllll}42 & 03 \quad 70 & 13.4 & 17 & \text { Jun } \\ 55\end{array}$

$\begin{array}{lllllll}41 & 24.5 & 70 & 32.2 & 12 & \text { JuI } & 55\end{array}$

42 14.1 7045.621 May 55

$\begin{array}{lllll}42 & 25.7 & 70 & 56.1 & 23 \\ 4 & 33 & \text { May } 55\end{array}$

$4233 \quad 7050 \quad 8$ May 55

$\begin{array}{llll}42 & 18.7 & 70 & 57.2 \quad 1 \\ 4 & 189 & 55\end{array}$

$\begin{array}{lllll}42 & 09.6 & 70 & 42.6 \quad 6 \text { Apr } 56\end{array}$

$4216.7 \quad 70 \quad 57.1 \quad 30$ Apr 55

$4236.67040 \quad 5$ Aug 55

$42 \quad 16.970 \quad 57.4 \quad 30$ Apr 55

$4259 \quad 7045.827$ Apr 55

$4256.3 \quad 7047.727$ Apr 55

$4259.2 \quad 70 \quad 45.8 \quad 26$ Apr 55

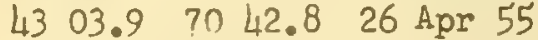

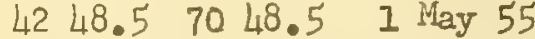

$4248.5 \quad 70 \quad 51.727$ Apr 55

$4248.970 \quad 48.525$ Apr 55 


\begin{tabular}{|c|c|c|c|c|c|c|c|}
\hline Station & Bottle & Irat & Long & Date & Lat & Long & Date \\
\hline 26 & $17889 \mathrm{E}$ & 4256.8 & 59.5 & 22 Apr & 4215.5 & 7048.4 & 28 Apr \\
\hline 26 & $17890 \mathrm{~N}$ & 4256.8 & 6959.5 & 22 Apr & 4218.0 & 7058.3 & $29 \mathrm{Apr}$ \\
\hline 26 & $17898 \mathrm{~N}$ & 4256.8 & 6959.5 & 22 Apr & 4201.1 & 7005.2 & 20 May \\
\hline 26 & $17899 \mathrm{~B}$ & 4256.8 & 6959.5 & 22 Apr & 4741.8 & 6957.1 & 26 Jun \\
\hline 26 & $17900 \mathrm{~N}$ & 4256.8 & 6959.5 & $22 \mathrm{Apr}$ & $4248^{\circ}$ & 7048.5 & $4 \mathrm{JuI}$ \\
\hline 27 & $17901 \mathrm{~B}$ & 4255.8 & 6933.8 & 22 Apr & 4204.9 & 7011 & 9 May \\
\hline 27 & $17904 \mathrm{~N}$ & 4255.8 & 6933.8 & 22 Apr & 4755.6 & 7032.8 & $30 \mathrm{Apr}$ \\
\hline 27 & $17906 \mathrm{~N}$ & 4255.8 & 6933.8 & 22 Арг & 4756.4 & 7034.1 & 22 May \\
\hline 27 & $17912 \mathrm{~N}$ & 4255.8 & 6933.8 & 22 Apr & 4147.1 & 7030.5 & 8 May \\
\hline 28 & & 4256.5 & 6858 & 22 Apr & No I & eturns & \\
\hline 29 & & 4254.4 & 6800.7 & 23 Apr & No $r$ & eturns & \\
\hline 30 & & 4252.5 & 6705 & 23 Apr & No & eturns & \\
\hline 31 & $17955 B$ & 4251 & 6629 & $23 \mathrm{Apr}$ & 4507.8 & 6634.2 & I Aug \\
\hline 32 & & 4249.5 & 6600.5 & 23 Apr & No $r$ & eturns & \\
\hline 33 & 17977B & 4244.2 & 6526 & $23 \mathrm{Apr}$ & 4456 & 6700.8 & $90_{c t}$ \\
\hline 33 & 17979B & 4244.2 & 6526 & 23 Apr & 4507.8 & 6634.2 & $31 \mathrm{Ju}$ \\
\hline 34 & $17993 B$ & 4239.2 & 6457 & 23 Apr & 4503 & 6647 & 5 Sep \\
\hline 34 & $17994 \mathrm{~N}$ & 4239.2 & 6457 & 23 Apr & 4503.7 & 6644 & 24 Jun \\
\hline 34 & $17985 \mathrm{~B}$ & 4239.2 & 6457 & $23 \mathrm{Apr}$ & 4504.6 & 6639 & 11 Sep \\
\hline 35 & $18005 B$ & 4215 & 6504 & $23 \mathrm{Apr}$ & 4201.6 & 7002.6 & 22 Oct \\
\hline 35 & $18008 \mathrm{~N}$ & 4215 & 6504 & 23 Apr & 4327 & 6516 & 2 JuI \\
\hline 36 & $18017 \mathrm{~B}$ & 4216 & 6531.5 & 23 Apr & 4341 & 6504 & 2 Dec \\
\hline 37 & $18028 \mathrm{~N}$ & 4315.5 & 6559.3 & 23 Apr & 4051.2 & 7230 & $150 \mathrm{ct}$ \\
\hline 38 & & 4215.8 & 6630.5 & $23 \mathrm{Apr}$ & No I & eturns & \\
\hline 39 & & 4216 & 6658.8 & $24 \mathrm{Apr}$ & No $r$ & eturns & \\
\hline 40 & & 4218 & 6733.4 & 24 Apr & No $r$ & eturns & \\
\hline 41 & & 4218 & 6801 & 24 Apr & No $r$ & eturns & \\
\hline 42 & $18086 \mathrm{~N}$ & 4219 & 6901.1 & 24 Apr & LI 20.9 & 7032.5 & 26 May \\
\hline 42 & $18087 \mathrm{~B}$ & 4219 & 6901.1 & $24 \mathrm{Apr}$ & 4728.3 & 7110 & 30 Jun \\
\hline 42 & $18088 \mathrm{~N}$ & 4219 & 6901.1 & 24 Apr & $40 \quad 45.4$ & 7246.8 & $11 \mathrm{Aug}$ \\
\hline 42 & $18089 \mathrm{~B}$ & 4219 & 6901.1 & 24 Apr & 3956.4 & 7404.3 & 13 Aug \\
\hline 42 & $18090 \mathrm{~N}$ & 4219 & 6901.1 & 24 Apr & 4101.8 & 7157.2 & $13 \mathrm{Jwn}$ \\
\hline 43 & $18096 \mathrm{~N}$ & 4216 & 6934.2 & $24 \wedge \mathrm{pr}$ & 4135.4 & 7038.6 & 6 Aug \\
\hline 43 & $18097 \mathrm{~B}$ & 4216 & 6934.2 & 24 Apr & 4122.4 & 7030.4 & $8 \mathrm{Aug}$ \\
\hline 43 & $18100 \mathrm{~N}$ & 4216 & 6934.2 & 24 Apr & 4044.5 & 7250 & 12 Jun \\
\hline 43 & $18101 \mathrm{~B}$ & 4216 & 6934.2 & 24 Apr & 3550 & 7537.1 & $13 \mathrm{Nov}$ \\
\hline 43 & $18103 \mathrm{~B}$ & 4216 & 6934.2 & 24 Apr & 4043 & 7255 & $10 \mathrm{JuI}$ \\
\hline 44 & $18108 \mathrm{~N}$ & 4215.3 & 6959 & 27 Apr & 4123.4 & 7030.3 & 20 Jun \\
\hline 44 & $18110 \mathrm{~N}$ & 4215.3 & 6959 & 27 Apr & 4125 & 7033 & 10 Jun \\
\hline 44 & $18115 \mathrm{~B}$ & 4215.3 & 6959 & 27 Apr & 4120.7 & 7040 & $20 \mathrm{Aug}$ \\
\hline 45 & 18119B & 4214.2 & 7012 & 27 Apr & 4149 & 6956.4 & 26 May \\
\hline 45 & $18125 \mathrm{~B}$ & 4214.2 & 7012 & 27 Apr & 4121 & 7001.6 & $22 \mathrm{JuJ}$ \\
\hline 45 & $18128 \mathrm{~N}$ & $42 \quad 14.2$ & 7012 & 27 Apr & 4149.2 & 6956.4 & $16 \mathrm{Aug}$ \\
\hline 46 & $18129 \mathrm{~B}$ & $\begin{array}{lll}42 & 01.9\end{array}$ & 6957.1 & 27 Apr & 4122.8 & 7030 & 31 May \\
\hline 46 & $18131 \mathrm{~B}$ & $\begin{array}{lll}42 & 01.9\end{array}$ & 6957.1 & 27 Apr & 4127.5 & 7039.9 & I4 Jun \\
\hline 46 & $18132 \mathrm{~N}$ & $\begin{array}{lll}42 & 01.9\end{array}$ & 6957.1 & 27 Apr & 4139.4 & 6959.1 & $28 \mathrm{May}$ \\
\hline 46 & $18133 \mathrm{~B}$ & $\begin{array}{ll}42 & 01.9\end{array}$ & 6957.1 & 27 Apr & 4722 & 7030 & 4 Ju1 \\
\hline
\end{tabular}




\begin{tabular}{|c|c|c|c|c|c|c|c|}
\hline Station & Bottle & Iat & Long & Date & Lat & Iong & Date \\
\hline 46 & $18134 \mathrm{~N}$ & 4201.9 & 69.57 .1 & 27 Apr & 4120.8 & 7030 & 20 May \\
\hline 46 & $18136 \mathrm{~N}$ & $\begin{array}{ll}42 & 01.9\end{array}$ & 6957.1 & 27 Apr & 4134 & 7039 & 4 Jun \\
\hline 46 & $18137 \mathrm{~B}$ & 4201.9 & 6957.1 & 27 Apr & 4044 & 72 5. 8 & 12 Jun \\
\hline 46 & $18138 \mathrm{~N}$ & 4201.9 & 6957.1 & 27 Apr & 4126.8 & 7055.3 & $20 \mathrm{Jul}$ \\
\hline 46 & $18139 \mathrm{~B}$ & 4201.9 & 6957.1 & 27 Apr & 4121 & 7050.2 & 6 Jun \\
\hline 46 & $1814 \mathrm{ON}$ & 4201.9 & 6957.1 & 27 Apr & 4120.9 & 7032.5 & 26 May \\
\hline 47 & $18142 \mathrm{~N}$ & 4144.6 & 6940 & 27 Apr & $40 \quad 42.7$ & 7255.7 & 15 Aug \\
\hline 47 & $18144 \mathrm{~N}$ & 4144.6 & 6940 & 27 Apr & 3918 & 7433 & 9 Oct \\
\hline 47 & $18151 \mathrm{~B}$ & 4144.6 & 6940 & 27 Apr & 4721 & 7047.4 & 8 Jun 5 \\
\hline 47 & $18152 \mathrm{~N}$ & 4144.6 & 6940 & 27 Apr & 4037.3 & 7318.5 & 14 Aug 5 \\
\hline 48 & $18154 \mathrm{~N}$ & 4744 & 6920 & 27 Apr & 4123 & 7030.3 & 31 May 5 \\
\hline 48 & $18160 \mathrm{~N}$ & 474 & 6920 & 27 Apr & 3712.8 & 7549 & 17 Nov 5 \\
\hline 49 & $18169 \mathrm{~B}$ & 4145 & 6857 & $27 \mathrm{Apr}$ & 4046 & 7245 & 21 Aug \\
\hline 50 & & 4147.2 & 6833 & 28 Apr & No & eturns & \\
\hline 51 & $18191 \mathrm{~B}$ & 4750.4 & 6804.6 & $28 \mathrm{Apr}$ & 4440 & 6546 & 1 Dec 5 \\
\hline 52 & & 4744 & 6655.5 & $28 \mathrm{Apr}$ & No I & eturns & \\
\hline 53 & & 4740.8 & 6629.8 & 28 Apr & No & eturns & \\
\hline 54 & & 414.7 & $\begin{array}{lll}66 & 03.8\end{array}$ & 28 Apr & No 1 & eturns & \\
\hline 55 & & 4145.2 & 6543 & $28 \mathrm{Apr}$ & No $r$ & eturns & \\
\hline 56 & & 4135 & 6554 & 28 Apr & No & e turns & \\
\hline 57 & $18262 \mathrm{IN}$ & 41 14.8 & 6616 & 28 Apr & 3532.5 & 7528 & 30 Sep \\
\hline 57 & $18272 \mathrm{~N}$ & 4714.8 & 6616 & 28 Apr & 4053.4 & 7220 & I4 Aug \\
\hline 58 & & 4114.2 & 6630.5 & 28 Apr & No 1 & eturns & \\
\hline 59 & & 4115.1 & 6659 & 28 Apr & No 1 & eturns & \\
\hline 60 & & 4114.2 & 6729 & 29 Apr & No & eturns & \\
\hline 61 & $18312 \mathrm{~N}$ & 4116.5 & 6803.4 & 29 Apr & 4132.6 & $70 \quad 36.7$ & 16 JuI \\
\hline 61 & $18316 \mathrm{~N}$ & 4116.5 & 6803.4 & 29 Apr & 4049.2 & 7231 & 19 Aug 5 \\
\hline 61 & $18320 \mathrm{~N}$ & 4116.5 & 6803.4 & 29 Apr & 4120.8 & 7030 & 16 JuI 5 \\
\hline 62 & $18328 \mathrm{~N}$ & 4117.2 & 6832.7 & 29 Apr & 4202 & 700.5 .7 & 28 oct 5 \\
\hline 63 & & 4114.3 & 6850.2 & 29 Apr & No & eturns & \\
\hline 64 & $18346 \mathrm{~N}$ & 4700 & 6849.3 & 29 Apr & 4120.9 & 7033 & 6 Jun \\
\hline 64 & $18348 \mathrm{~N}$ & 4100 & 6849.3 & 29 Apr & 4138.3 & 7038.6 & 30 Jun \\
\hline 64 & $18350 \mathrm{~N}$ & 4700 & 6849.3 & 29 Apr & 3554.1 & 7535.4 & 工4 Sep \\
\hline 64 & $18352 \mathrm{~N}$ & 4100 & 6849.3 & 29 Apr & 3521 & 7530 & 15 Sep \\
\hline 64 & $18354 \mathrm{~N}$ & 4700 & 6849.3 & 29 Apr & 4038.3 & 7318.6 & 4 Sep \\
\hline 64 & $18355 \mathrm{~B}$ & 4100 & 6849.3 & 29 Apr & 4130 & 7120 & 25 Jun \\
\hline 65 & & 4043.6 & 6845.6 & 29 Apr & No & eturns & \\
\hline 66 & & 4040 & 6823.5 & 30 Apr & No $I$ & eturns & \\
\hline 67 & $18383 B$ & 4939.5 & 6753.5 & 30 Apr & 3730 & 7538.6 & 4 Oct \\
\hline 67 & $18389 \mathrm{~B}$ & 4039.5 & 6753.5 & 30 Apr & 4728.8 & 7116.6 & $14 \mathrm{Aug}$ \\
\hline 68 & 18393B & 4039.4 & 6726.4 & 30 Apr & 3515.8 & 7531 & 7 Oct \\
\hline 68 & $18402 \mathrm{~N}$ & 4039.4 & 6726.4 & 30 Apr & 4038.3 & 7311 & 16 Aug \\
\hline 69 & & 4040.5 & 6700.7 & 30 Apr & No $r$ & eturns & \\
\hline 70 & $18420 \mathrm{~N}$ & 4044.3 & 6642.9 & 30 Apr & 3820 & 7505 & 23 Sep \\
\hline
\end{tabular}




\begin{tabular}{|c|c|c|c|c|c|c|c|}
\hline Station & Bottle & Lat & Long & Dete & Lat & Long & Date \\
\hline 71 & $18432 \mathrm{~N}$ & 4101.5 & 6632 & $30 \mathrm{Apr}$ & 4053 & 7228.6 & 28 Aug 55 \\
\hline 71 & $18436 \mathrm{~N}$ & 4101.5 & 6632 & $30 \mathrm{Apr}$ & 3954.2 & 7404.7 & 12 Aug 55 \\
\hline 71 & $18439 \mathrm{~B}$ & 4701.5 & 6632 & 30 Apr & $40 \quad 02.2$ & 7403 & 22 Sep 55 \\
\hline 72 & & 4133.6 & 6617 & 1 May & \multicolumn{3}{|c|}{ No r eturns } \\
\hline 73 & & 4202.6 & 6603 & 1 May & \multicolumn{3}{|c|}{ No returns } \\
\hline 74 & & 4226 & 6539 & 1 May & \multicolumn{3}{|c|}{ No returns } \\
\hline 75 & $18482 \mathrm{~N}$ & 4245.6 & 6528 & $1 \mathrm{May}$ & 4505 & 6628 & 12 Oct 55 \\
\hline 75 & $18487 B$ & 4245.6 & 6528 & 1 May & 4147.7 & 7001.2 & 23 Nov 55 \\
\hline 76 & $18498 \mathrm{~N}$ & 4257 & 6520.5 & $1 \mathrm{May}$ & 4436 & 6654.5 & $30 \mathrm{JuZ} 55$ \\
\hline 77 & & 4258 & 6541 & 1 May & \multicolumn{3}{|c|}{ No returns } \\
\hline 78 & $18518 \mathrm{~N}$ & 4258 & 6600 & I May & 4348 & 6609 & 21 May 55 \\
\hline 78 & $18521 \mathrm{~B}$ & 4258 & 6600 & $1 \mathrm{May}$ & 4434.5 & 6555 & $31 \mathrm{May} 55$ \\
\hline 78 & $18522 \mathrm{~N}$ & 4258 & 6600 & 1 May & 4320.3 & 6537.3 & 29 JuI 55 \\
\hline 79 & $18526 \mathrm{~N}$ & 4241 & 6548.2 & 1 May & 4329 & 6529.5 & 6 Jun 55 \\
\hline 79 & $18531 \mathrm{~B}$ & 4241 & 6548.2 & 1 May & 4307.2 & 7051.5 & 28 Apr 56 \\
\hline 79 & $18532 \mathrm{~N}$ & 4247 & 6548.2 & 1 May & 4445.9 & $6 6 \longdiv { 4 4 . 2 }$ & $3 \mathrm{Aug} 55$ \\
\hline 79 & $18533 \mathrm{~B}$ & 4241 & 6548.2 & I May & 4506 & 6630 & 5 Juบ 55 \\
\hline 79 & $18534 \mathrm{~N}$ & 4247 & 6548.2 & 1 May & 4433 & 6602 & $10 \mathrm{Aug} 55$ \\
\hline 80 & & 4227 & 6539 & 1 May & \multicolumn{3}{|c|}{ No returns } \\
\hline 81 & $18552 \mathrm{~N}$ & 4215.2 & 6531 & 1 May & 4401 & 6837 & 28 oct 55 \\
\hline 82 & & 4207 & 6545 & 1 May & \multicolumn{3}{|c|}{ No returns } \\
\hline 83 & & 4155.8 & 6602.5 & 2 May & \multicolumn{3}{|c|}{ No returns } \\
\hline 84 & & 4746.3 & 6617.4 & 2 May & \multicolumn{3}{|c|}{ No returns } \\
\hline 85 & & 4132 & 6634 & 2 May & \multicolumn{3}{|c|}{ No returns } \\
\hline 86 & & 4118.5 & 6652 & 2 May & \multicolumn{3}{|c|}{ No returns } \\
\hline 87 & & 4112.3 & 6715 & 2 May & \multicolumn{3}{|c|}{ No returns } \\
\hline 88 & & 4103 & 6730 & $2 \mathrm{May}$ & \multicolumn{3}{|c|}{ No returns } \\
\hline 89 & & 4052.5 & 6748 & 2 May & \multicolumn{3}{|c|}{ No retums } \\
\hline 90 & $18668 \mathrm{~N}$ & 4040.6 & 6808.5 & 2 May & 5630 & 0707 & 25 Aug 56 \\
\hline 91 & & 4036.8 & 6811.4 & 2 May & \multicolumn{3}{|c|}{ No returns } \\
\hline 92 & & 4033.2 & 6751.4 & 2 May & \multicolumn{3}{|c|}{ No returns } \\
\hline 93 & $18694 N$ & 4031 & 6729 & 2 May & 4735 & 7050 & 6 Aug 55 \\
\hline 93 & 18703B & 4031 & 6729 & $2 \mathrm{May}$ & 4047 & 7248 & 17 Aug 55 \\
\hline 94 & $18705 \mathrm{~B}$ & 4032 & 6752.8 & 2 May & 4039.5 & 7305 & 28 sep 55 \\
\hline 94 & I8710N & 4032 & 6752.8 & 2 May & 3903 & 7445.4 & 14 Sep 55 \\
\hline 94 & 1871]B & 4032 & 6752.8 & 2 May & 3903 & 7445.4 & 25 Sep 55 \\
\hline 95 & & 4029.8 & 6817.8 & 2 May & \multicolumn{3}{|c|}{ No returns } \\
\hline 96 & & 4027.8 & 6840 & 2 May & \multicolumn{3}{|c|}{ No returns } \\
\hline 97 & $18748 \mathrm{~N}$ & 4024.7 & 6909 & 3 May & 6047 & 0048 & 12 Oct 56 \\
\hline 98 & $18753 \mathrm{~B}$ & 4029.4 & 6929.3 & 3 May & 3903 & 74.45 .4 & 19 Sep 55 \\
\hline 98 & $18754 \mathrm{~N}$ & 4029.4 & 6929.3 & 3 May & 4051 & 7226.8 & 7 Aug 5 \\
\hline 98 & $18756 \mathrm{~N}$ & 4029.4 & 6929.3 & 3 May & 3526.7 & 7528.9 & 28 Sep 5 \\
\hline 98 & $18758 \mathrm{~N}$ & 4029.4 & 6929.3 & 3 May & 4052 & 7224 & $15 \mathrm{Aug} 55$ \\
\hline 98 & $18759 \mathrm{~B}$ & 4029.4 & 6929.3 & 3 May & 3956.4 & 7404.3 & 14 Aug 55 \\
\hline 98 & $18761 \mathrm{~B}$ & 4029.4 & 6929.3 & $3 \mathrm{May}$ & 4104 & 7156.9 & 14 Aug 55 \\
\hline
\end{tabular}


Released

\begin{tabular}{|c|c|c|c|c|}
\hline Station & Bottie & Lat & Long & Date \\
\hline 99 & & 4031.2 & 6945.8 & 3 Мау \\
\hline 100 & 18777B & 4038.5 & 7007.7 & 3 May \\
\hline 100 & $18778 \mathrm{~N}$ & 4038.5 & 7007.7 & 3 May \\
\hline 100 & $18781 \mathrm{~B}$ & 4038.5 & $7007 . ?$ & 3 May \\
\hline 100 & $18782 \mathrm{~N}$ & 4038.5 & 7007.7 & $3 \mathrm{May}$ \\
\hline 101 & $18789 \mathrm{~B}$ & 4056 & 7030.5 & 3 May \\
\hline 101 & $18791 B$ & 4056 & 7030.5 & 3 May \\
\hline 101 & $18792 \mathrm{~N}$ & 4056 & 7030.5 & $3 \mathrm{May}$ \\
\hline 101 & $18795 \mathrm{~B}$ & 4056 & 7030.5 & 3 Мау \\
\hline 101 & $18796 \mathrm{~N}$ & 4056 & 7030.5 & 3 May \\
\hline 101 & 18799B & 4056 & 7030.5 & 3 May \\
\hline 102 & $18812 B$ & 4111.7 & 7050 & \\
\hline
\end{tabular}

\section{Recovered}

Lat Iong Date

No returns

$4135.67039 \quad 24$ Jun 55

$4039.6 \quad 7305 \quad 13$ Jun 55

$\begin{array}{llll}41 & 28.8 & 70 & 45.1 \quad 1 \text { Jun } 55\end{array}$

$4120.8 \quad 7029 \quad 24$ May 55

$41 \quad 18.470 \quad 47.328$ May 55

$\begin{array}{lllll}47 & 20.9 & 70 & 32.5 & 24 \\ 4 a y & 55\end{array}$

$4120 \quad 7044 \quad 27$ JuI 55

$4718.7 \quad 7048 \quad 29$ May 55

$\begin{array}{llllll}41 & 24.1 & 70 & 30.7 \quad 10 & \text { Apr } 56\end{array}$

41. $21 \quad 70 \quad 30.5 \quad 25$ May 55

4130.17106 .529 May 55 
Released

\begin{tabular}{|c|c|c|c|c|}
\hline Station & Bottle & Lat & Iong & Date \\
\hline 1 & $18816 \mathrm{~N}$ & 4117.3 & 7100 & $16 \mathrm{May}$ \\
\hline 1 & $18818 \mathrm{~N}$ & 4117.3 & 7100 & $16 \mathrm{May}$ \\
\hline 1 & $18820 \mathrm{~N}$ & 4117.3 & 7100 & $16 \mathrm{May}$ \\
\hline 1 & $18822 \mathrm{~N}$ & 417.3 & 7100 & 16 May \\
\hline 2 & 18825B & 4039.5 & 7030 & $16 \mathrm{May}$ \\
\hline 2 & $18829 B$ & 4039.5 & 7030 & 16 Маy \\
\hline 2 & $18831 B$ & 4039.5 & 7030 & $16 \mathrm{May}$ \\
\hline 3 & $18844 \mathrm{~N}$ & 4000 & 7000 & 16 May \\
\hline 4 & $18853 \mathrm{~B}$ & 4000 & $\begin{array}{ll}69 & 05.3\end{array}$ & 16 May \\
\hline 5 & & 3959 & 6759 & 17 May \\
\hline 6 & & 4702 & 6723.5 & 17 May \\
\hline 7 & & 4200 & 6658 & 17 May \\
\hline 8 & $18903 B$ & 4237.2 & 6621 & 17 May \\
\hline 8 & $18906 \mathrm{~N}$ & 4237.2 & 6621 & 17 May \\
\hline 9 & $18912 \mathrm{~N}$ & 4315 & 6606.8 & $18 \mathrm{May}$ \\
\hline 9 & $18919 \mathrm{~B}$ & 4315 & 6606.8 & $18 \mathrm{May}$ \\
\hline 9 & $18920 \mathrm{~N}$ & 4315 & $\begin{array}{lll}66 & 06.8\end{array}$ & $18 \mathrm{May}$ \\
\hline 10 & $18926 \mathrm{~N}$ & 4319 & 6721.5 & $18 \mathrm{May}$ \\
\hline 11 & $18940 \mathrm{~N}$ & 4352 & 6620 & $18 \mathrm{May}$ \\
\hline 11 & $18947 \mathrm{~B}$ & 4352 & 6620 & $18 \mathrm{May}$ \\
\hline 11 & $18944 \mathrm{~N}$ & 4352 & 6620 & $18 \mathrm{May}$ \\
\hline 12 & $18947 \mathrm{~B}$ & 4420 & 6730 & $18 \mathrm{May}$ \\
\hline 12 & $18948 \mathrm{~N}$ & W4 20 & 6730 & $18 \mathrm{May}$ \\
\hline 12 & $18956 \mathrm{~N}$ & 4420 & 6730 & $18 \mathrm{May}$ \\
\hline 13 & & 4423.8 & 6630.6 & 19 May \\
\hline 14 & & 4347 & 6712 & $19 \mathrm{May}$ \\
\hline 15 & $18981 \mathrm{~B}$ & 4329.3 & $66 \quad 11.2$ & $19 \mathrm{May}$ \\
\hline 15 & $18988 \mathrm{~N}$ & 4329.3 & 6611.2 & 19 May \\
\hline 16 & $19004 \mathrm{~N}$ & 4330.3 & 6803 & 19 May \\
\hline 17 & & 4331 & 6900 & 20 :lay \\
\hline 18 & & 4331 & 7003.4 & 20 May \\
\hline 19 & $19130 \mathrm{~N}$ & 4257.8 & 7022.3 & $20 \mathrm{May}$ \\
\hline 20 & $19043 \mathrm{~B}$ & 4248 & 6855 & 20 May \\
\hline 21 & $19054 \mathrm{~N}$ & 4248.9 & 6753.8 & 20 May \\
\hline 21 & $19064 \mathrm{~N}$ & 4248.9 & 6753.8 & 20 May \\
\hline 22 & $19067 \mathrm{~B}$ & 4247.4 & 6657.2 & $20 \mathrm{May}$ \\
\hline 22 & $19069 \mathrm{~B}$ & 4247.4 & 6657.2 & $20 \mathrm{May}$ \\
\hline 22 & $19072 \mathrm{~N}$ & 4247.4 & 6657.2 & $20 \mathrm{May}$ \\
\hline 23 & & 4244 & 6602 & $21 \mathrm{May}$ \\
\hline 24 & & 4233 & 6438.8 & $21 \mathrm{May}$ \\
\hline 25 & & 4200 & $6 4 \longdiv { 4 2 }$ & 21 May \\
\hline
\end{tabular}

Recovered

Iat Long Date

$4129.27044 \quad 5$ Jun 55

$4113.97134 .6 \quad 10$ Jun 55

$4138.17020 \quad 31 \mathrm{May} 55$

$4138.1 \quad 7017 \quad 31$ May 55

$4725 \quad 7055.8 \quad 6$ Jun 55

$4111.5 \quad 7133.9 \quad 10$ Jun 55

$4113.5 \quad 7133.8 \quad 9$ Jun 55

$\begin{array}{llllll}39 & 31.2 & 74 & 16.5 & 19 & \text { Sep } 55\end{array}$

$4047.27240 \quad 4$ Sep 55

No returns

No returns

No returns

$4340 \quad 6505 \quad 12$ Jun 55

$4508.5 \quad 6616 \quad 12 \mathrm{Sep} 55$

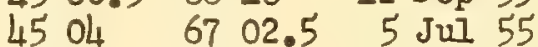

$\begin{array}{lllll}43 & 38 & 66 & 03 & 16 \\ 4 & \text { Nov } 55\end{array}$

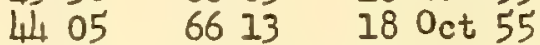

$4241.57047 .525 \mathrm{Nov} 55$

$\begin{array}{lllll}44 & 46 & 65 & 36.5 & 14 \\ \text { Jun } & 55\end{array}$

$4340.6 \quad 7010 \quad 21$ Aug 55

$4507.2 \quad 6630 \quad 22$ Jun 55

$4457.5 \quad 65$ I4 31 Oct 55

$4336.47013 \quad 14$ Jun 55

$4416 \quad 6608 \quad 10$ Sep 55

No returns

No returns

$4508 \quad 6453 \quad 29$ Aug 55

45 or 6704.631 JuI 55

$4457 \quad 6654 \quad 7$ Sep 55

No returns

No r eturns

$3920.6 \quad 7428 \quad 7$ oct 55

$4452 \quad 6656 \quad 22$ Oct 55

$4514 \quad 6603.5 \quad 14$ Sep 55

$4449.3 \quad 6531 \quad 25$ Sep 55

$\begin{array}{lll}4338 & 7013 \quad 8 \text { oct } 56\end{array}$

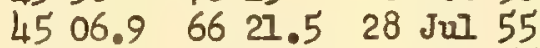

$4449.5 \quad 66 \quad 58 \quad 30$ JuI 55

No returns

No returns

No returas 


\begin{tabular}{|c|c|c|c|c|c|c|c|}
\hline Station & Bottle & Lat & Long & Date & Lat & Iong & Date \\
\hline 26 & $19114 \mathrm{~N}$ & 4758.2 & 6603 & 21 May & 4447 & 6535 & 1 Nov 55 \\
\hline 26 & $19122 \mathrm{~N}$ & 4758.2 & 6603 & 21 May & 4326.3 & 6537.6 & 28 Jun 55 \\
\hline 27 & & 4204.2 & 6658 & 21 May & No & eturns & \\
\hline 28 & $19146 \mathrm{~N}$ & 4202 & 6801.6 & 22 May & $4327 . ?$ & 6529.6 & 17 Jul 5 \\
\hline 29 & $19150 \mathrm{~N}$ & 4233.3 & 7031.7 & 22 May & 4327.5 & 6539 & 18 Aug 5 \\
\hline 29 & $19154 \mathrm{~N}$ & 4233.3 & 7031.7 & 22 May & $\begin{array}{ll}42 & 03.7\end{array}$ & 7006 & 12 Jun \\
\hline 29 & $19158 \mathrm{~N}$ & 4233.3 & 7031.7 & 22 May & 4204.3 & $\begin{array}{lll}70 & 07.8\end{array}$ & 15 Jun 5 \\
\hline 30 & & 4210.7 & 70.02 & 22 May & No & eturns & \\
\hline 31 & & 4751.8 & 6936.9 & 22 May & No & eturns & \\
\hline 32 & 19189B & 4149 & 6855.5 & 23 May & W4 56.5 & 6517 & 20 Sep 5 \\
\hline 33 & & 4144.7 & 6804 & 23 May & No r & turns & \\
\hline 34 & $19210 \mathrm{~N}$ & 4132 & 6932.5 & 23 Мay & 4120.8 & 7034 & $8 \mathrm{JuZ}$ \\
\hline 34 & $19211 \mathrm{~B}$ & 4732 & 6932.5 & $23 \mathrm{May}$ & 4728 & 7123.4 & $14 \mathrm{Aug}$ \\
\hline 34 & $19219 \mathrm{~B}$ & 4132 & 6932.5 & $23 \mathrm{May}$ & 4120.8 & 7030 & $15 \mathrm{JuI}$ \\
\hline 35 & & 4118.5 & 6855.2 & 23 Мау & No & eturns & \\
\hline 36 & & 4727 & 6802 & 23 May & No & eturns & \\
\hline 37 & $19248 \mathrm{~N}$ & 4132.8 & 6655.8 & $24 \mathrm{May}$ & 4717 & 7215.7 & 15 Aug \\
\hline 37 & $19251 B$ & 4732.8 & 6555.8 & 24 May & 4730 & 7107.6 & $25 \mathrm{Aug}$ \\
\hline 37 & 192558 & 4132.8 & 6655.8 & $24 \mathrm{May}$ & 4730 & 7103 & $26 \mathrm{Jul}$ \\
\hline 37 & $19256 \mathrm{~N}$ & 4132.8 & 6655.8 & 24 May & 4735 & 7051.5 & $19 \mathrm{JuI} 5$ \\
\hline 38 & & 4725.8 & 6551.4 & 24 May & No & eturns & \\
\hline 39 & & 4700.9 & 6615.9 & $24 \mathrm{May}$ & No & eturns & \\
\hline 40 & $19282 \mathrm{~N}$ & L17 03.6 & 6700.9 & $24 \mathrm{May}$ & 4058 & 7207.8 & 15 Aug \\
\hline 40 & $19287 B$ & LI 03.6 & 6700.9 & $24 \mathrm{May}$ & 4733.7 & 7113.2 & 4 Aug \\
\hline 40 & $19288 \mathrm{~N}$ & 4703.6 & 6700.9 & 24 May & 4057.6 & 7209 & $15 \mathrm{Aug}$ \\
\hline 40 & I9290N & 4103.6 & 6700.9 & $24 \mathrm{May}$ & 4036.8 & 7324.4 & 15 Aug 5 \\
\hline 40 & 19291B & 4103.6 & 6700.9 & 24 May & 3931 & 3107 & 2 Aug 5 \\
\hline 41 & & 4102 & 6909.4 & 24 May & No & eturns & \\
\hline 42 & $19306 \mathrm{~N}$ & 4030.4 & 6903.2 & $25 \mathrm{May}$ & 4005 & 7402.4 & $14 \mathrm{Aug}$ \\
\hline 42 & $19310 \mathrm{~N}$ & 4030.4 & 6903.2 & $25 \mathrm{May}$ & 4046 & 7245 & 9 Aug \\
\hline 42 & $19311 \mathrm{~B}$ & 4030.4 & 6903.2 & $25 \mathrm{May}$ & 4035 & 7334 & $21 \mathrm{Aug}$ \\
\hline 42 & $19312 \mathrm{~N}$ & 4030.4 & 6903.2 & 25 May & 4033.1 & 7356 & $15 \mathrm{Aug}$ \\
\hline 42 & $19313 \mathrm{~B}$ & 4030.4 & 6903.2 & $25 \mathrm{May}$ & 4047.3 & 7240 & If Aug \\
\hline 42 & $19316 \mathrm{~N}$ & 4030.4 & 6903.2 & $25 \mathrm{May}$ & 4143 & 7037 & 22 JuI \\
\hline 43 & $19327 \mathrm{~B}$ & 4028.2 & 6800.2 & $25 \mathrm{May}$ & 3937.2 & 7411.7 & 20 Sep 5 \\
\hline 44 & & 4031.4 & 6655.8 & $25 \mathrm{May}$ & No & eturns & \\
\hline 45 & & 4031.3 & 6631.6 & 25 May & No $r$ & eturns & \\
\hline 46 & & 3950 & 67 U4 & 25 May & No & e turns & \\
\hline 47 & $19372 N$ & $10 \quad 58.5$ & 6757.8 & 26 May & 4048 & 7242.7 & 21 Aug \\
\hline 47 & $19376 \mathrm{~N}$ & $40 \quad 58.5$ & 6757.8 & 26 May & 4730.5 & 7105 & 8 Jun \\
\hline 48 & $19318 \mathrm{~N}$ & 3945.8 & 6828.8 & $26 \mathrm{May}$ & 3919.5 & 74.30 & 8 oct \\
\hline 49 & & 4057.4 & 6900.8 & 26 May & No & eturns & \\
\hline 50 & $19392 \mathrm{~N}$ & 3946 & 6930.4 & 27 Мay & 405.5 & 7225 & $15 \mathrm{Aug}$ \\
\hline 51 & $19414 \mathrm{~N}$ & $40 \quad 42.3$ & 7006.2 & $27 \mathrm{May}$ & 4052 & 7223.5 & 13 Jun \\
\hline 51 & 194158 & $40 \quad 42.3$ & $70 \quad 06,2$ & 27 Мау & 3513.7 & 7531.6 & 17 Sep \\
\hline
\end{tabular}


Released

\begin{tabular}{llllll} 
Station & Bottle & Lat & Iong & Date \\
\cline { 4 - 6 } 51 & 19416N & 4042.3 & 7006.2 & 27 May \\
51 & $19478 \mathrm{~N}$ & 4042.3 & 7006.2 & $27 \mathrm{May}$ \\
51 & $19423 \mathrm{~B}$ & 4042.3 & 7006.2 & $27 \mathrm{May}$ \\
51 & $19424 \mathrm{~N}$ & 4042.3 & 7006.2 & $27 \mathrm{May}$ \\
52 & & 3949.6 & 7033.1 & $27 \mathrm{May}$ \\
53 & $1944 \mathrm{~N}$ & 4042 & 7116 & $27 \mathrm{May}$ \\
53 & $19442 \mathrm{~N}$ & 4042 & 7116 & $27 \mathrm{May}$ \\
54 & & 4002.8 & 7157.2 & $28 \mathrm{May}$ \\
55 & $19467 \mathrm{~B}$ & 4100.2 & 7137.6 & $28 \mathrm{May}$ \\
55 & $19468 \mathrm{~N}$ & 4700.2 & 7137.6 & $28 \mathrm{May}$ \\
56 & $19476 \mathrm{~N}$ & 4112.8 & 7115.5 & $28 \mathrm{May}$ \\
56 & $19479 \mathrm{~B}$ & 4112.8 & 7115.5 & $28 \mathrm{May}$ \\
56 & $19481 \mathrm{~B}$ & 4712.8 & 7115.5 & $28 \mathrm{May}$ \\
56 & $19482 \mathrm{~N}$ & 4112.8 & 7115.5 & $28 \mathrm{May}$ \\
56 & $19483 \mathrm{~B}$ & 4112.8 & 7115.5 & $28 \mathrm{May}$ \\
56 & $19484 \mathrm{~N}$ & 4112.8 & 7115.5 & $28 \mathrm{May}$
\end{tabular}

Recovered

Iat Iong Date

$40 \quad 46.5 \quad 72 \quad 42.8 \quad 12$ Jun 55 $\begin{array}{llllll}36 & 55.8 & 76 & 10.7 & 20 & \text { Sep } 55\end{array}$ $\begin{array}{llll}35 & 25 & 7529 & 7 \text { Nov } 56\end{array}$ $4046.5 \quad 7242.8 \quad 12$ Jun 55 No returns

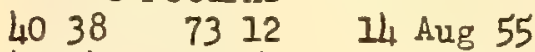

$4034.7 \quad 7350 \quad 9$ Sep 55 No returns

$\begin{array}{llll}36 & 05 & 7542 & 18 \text { Sep } 55\end{array}$

$\begin{array}{llllll}38 & 36.5 & 75 & 03.6 \quad 14 & \text { Aug } 55\end{array}$

$\begin{array}{lllllll}38 & 30 & 75 & 03.1 & 24 & \text { Sep } 55\end{array}$

$\begin{array}{lllll}38 & 20 & 75 & 05 & 17\end{array}$ Aug 55

$\begin{array}{lllllll}40 & 38.1 & 73 & 11.8 & 13 & \text { Jun } 55\end{array}$

$4171 \quad 7134 \quad 9$ Jun 55

$\begin{array}{llllll}40 & 38.1 & 73 & 11.8 & 12 & \text { Jun } 55\end{array}$

41197349 Jun 56 


\begin{tabular}{|c|c|c|c|c|c|c|c|}
\hline \multirow[b]{2}{*}{ Station } & \multicolumn{4}{|c|}{ Released } & \multicolumn{3}{|c|}{ Recovered } \\
\hline & Bottle & Lat & Long & Date & Lat & Long & Date \\
\hline 1 & & 4117.5 & 7100 & $20 \mathrm{Feb}$ & No: & eturns & \\
\hline 2 & & 4050.5 & 7101 & $20 \mathrm{Feb}$ & No: & etums & \\
\hline 3 & & 4024 & 7100.5 & $20 \mathrm{Feb}$ & No : & eturns & \\
\hline 4 & & 4000 & 7100 & $20 \mathrm{Feb}$ & No & etarns & \\
\hline 5 & & 3958 & 7022 & $21 \mathrm{Feb}$ & No ? & eturns & \\
\hline 6 & & 3956.5 & 6945 & $21 \mathrm{Feb}$ & No & eturns & \\
\hline 7 & & 3956.3 & 6857 & $21 \mathrm{Feb}$ & No ? & e turns & \\
\hline 8 & & 3957 & 6800 & 21 Feb & No ? & eturns & \\
\hline 9 & & $40 \quad 26.2$ & 6742.5 & $21 \mathrm{Feb}$ & No ? & eturns & \\
\hline 10 & & 4053.5 & 6726 & 21 Feb & No I & eturns & \\
\hline 17 & & 4123 & 6716 & 22 Feb & No & eturns & \\
\hline 12 & & 4755.3 & 6701 & 22 Feb & No & eturns & \\
\hline 13 & $19639 B$ & 4218.5 & 6645 & 22 Feb & 47.30 & 7102.5 & 5 sep 56 \\
\hline 14 & & 4245 & 6632 & $22 \mathrm{Feb}$ & No & eturns & \\
\hline 15 & $19662 \mathrm{~N}$ & 4318.8 & 6613.3 & $22 \mathrm{Feb}$ & 4334 & 6547 & 13 May 56 \\
\hline 16 & 196718 & 4344 & 6632 & $22 \mathrm{Feb}$ & 4458 & 6703 & 27 JuI 56 \\
\hline 17 & & 4356.7 & 6641.2 & $22 \mathrm{Feb}$ & No & eturns & \\
\hline 18 & $19694 N$ & 4350 & 6730 & $23 \mathrm{Feb}$ & 4423.5 & 6612.7 & 5 May 56 \\
\hline 19 & $20344 N$ & 1344 & 6805.5 & $23 \mathrm{Feb}$ & 4218 & 7053 & 26 oct 56 \\
\hline 19 & $20350 \mathrm{~N}$ & 4344 & 6805.5 & $23 \mathrm{Feb}$ & 4344.2 & 7001.3 & $28 \mathrm{JuZ} 56$ \\
\hline 20 & $20356 \mathrm{~N}$ & 4334.6 & 6834.9 & $23 \mathrm{Feb}$ & 4456 & 6658 & 22 Jun 56 \\
\hline 20 & 203618 & 4334.6 & 6834.9 & $23 \mathrm{~F} \in \mathrm{b}$ & $\begin{array}{lll}42 & 01.9\end{array}$ & 7005.6 & 23 Nov 56 \\
\hline 20 & $20364 \mathrm{~N}$ & 4334.6 & 6834.9 & $23 \mathrm{Feb}$ & 4121 & 7027 & 1 Nov 56 \\
\hline 21 & 20365B & 4330.5 & 6920.7 & $23 \mathrm{Feb}$ & 4138.1 & 7020 & 29 May 56 \\
\hline 27 & $20369 B$ & 4330.5 & 6920.7 & 23 Feb & $4 1 \longdiv { 4 0 . 5 }$ & 7043 & 1 Jun 56 \\
\hline 21 & $20370 \mathrm{~N}$ & 4330.5 & 6920.7 & $23 \mathrm{Feb}$ & 4150 & 6956.6 & 26 Jun 56 \\
\hline 27 & $20373 B$ & 4330.5 & 6920.7 & $23 \mathrm{Feb}$ & 4723 & 7030.3 & 22 Jun 56 \\
\hline 21 & $20374 N$ & 4330.5 & 6920.7 & $23 \mathrm{Feb}$ & 4121.2 & 7046.1 & 18 May 56 \\
\hline 21 & $20376 N$ & 4330.5 & 6920.7 & 23 Feb & 4123 & 7045 & 25 Juב 56 \\
\hline 22 & $20379 B$ & 4326.3 & 7004.5 & $23 \mathrm{Feb}$ & 4739 & 7010.5 & 5 Jun 56 \\
\hline 22 & $20381 B$ & 4326.3 & 7004.5 & $23 \mathrm{Feb}$ & 4136 & 7039 & $13 \mathrm{JuI} 56$ \\
\hline 23 & $19704 \mathrm{~N}$ & 4254.8 & 7026.5 & 23 Feb & 4204.4 & 7014.3 & 1 Jun 56 \\
\hline 23 & $19706 \mathrm{~N}$ & 4254.8 & 7026.5 & $23 \mathrm{Feb}$ & 4151.7 & 6957.1 & 21 Mar 5 \\
\hline 23 & $19707 \mathrm{~B}$ & 4254.8 & 7026.5 & $23 \mathrm{Feb}$ & 4144.2 & 7020 & 11 Aug 5 \\
\hline 23 & $19709 \mathrm{~B}$ & 4254.8 & 7026.5 & $23 \mathrm{Feb}$ & $\begin{array}{lll}42 & 01.9\end{array}$ & 7037.5 & 19 Apr 5 \\
\hline 24 & $19715 B$ & 4253.3 & 6943.8 & $23 \mathrm{Feb}$ & $\begin{array}{lll}42 & 03.8\end{array}$ & 7014.8 & 2 Apr 5 \\
\hline 24 & $19717 \mathrm{~B}$ & 4253.3 & 6943.8 & $23 \mathrm{Feb}$ & $\begin{array}{ll}42 & 04.8\end{array}$ & 7013.3 & 25 Mar 5 \\
\hline 24 & $19718 \mathrm{~N}$ & 4253.3 & 6943.8 & $23 \mathrm{Feb}$ & 4204.8 & 7013.3 & 2 Apr 5 \\
\hline 24 & 19719B & 4253.3 & 6943.8 & $23 \mathrm{Feb}$ & 4204.3 & 7008 & 28 Мау 56 \\
\hline 24 & $19721 B$ & 4253.3 & 6943.8 & $23 \mathrm{Feb}$ & 4157.5 & 7004.7 & 7 Apr 56 \\
\hline 24 & 19723B & 4253.3 & 6943.8 & $23 \mathrm{Feb}$ & 4204 & 7014.7 & 21 Mar 56 \\
\hline
\end{tabular}


Released

\begin{tabular}{|c|c|c|c|c|}
\hline Station & Bottle & Lat & Long & Date \\
\hline 25 & $19729 B$ & 4248.5 & 6914 & $23 \mathrm{Feb}$ \\
\hline 26 & & 4248 & 6827 & $24 \mathrm{Feb}$ \\
\hline 27 & & 4247 & 6738 & $24 \mathrm{Feb}$ \\
\hline 28 & $19806 \mathrm{~N}$ & 4246.5 & 6658 & $24 \mathrm{Feb}$ \\
\hline 28 & $19808 \mathrm{~N}$ & 4246.5 & 6658 & $24 \mathrm{Feb}$ \\
\hline 29 & & 4242.5 & 6615 & $24 \mathrm{Feb}$ \\
\hline 30 & & $4238^{\circ}$ & 6542 & $24 \mathrm{Feb}$ \\
\hline 31 & & 4235.3 & 6451.5 & 24 Feb \\
\hline 32 & & 4222.5 & 6503 & 24 Feb \\
\hline 33 & & 4208.8 & 6525.1 & $24 \mathrm{Feb}$ \\
\hline 34 & & 4207.8 & 6553.5 & $24 \mathrm{Feb}$ \\
\hline 35 & & 4208 & 6632 & $25 \mathrm{Feb}$ \\
\hline 36 & & 4217 & 6709 & $25 \mathrm{Feb}$ \\
\hline 37 & 19969B & 4213 & 6746 & $25 \mathrm{Feb}$ \\
\hline 38 & I9980N & 4209.4 & 6825.2 & $25^{\circ} \mathrm{Feb}$ \\
\hline 38 & $19984 \mathrm{~N}$ & $\begin{array}{ll}42 & 09.4\end{array}$ & 6825.2 & $25 \mathrm{Feb}$ \\
\hline 39 & $19990 \mathrm{~N}$ & 4220.5 & 6901 & $25 \mathrm{Feb}$ \\
\hline 40 & $20014 \mathrm{~N}$ & 4231.8 & 6946 & $25 \mathrm{Feb}$ \\
\hline 40 & $20015 \mathrm{~B}$ & 4231.8 & 6946 & 25 Feb \\
\hline 40 & $20017 \mathrm{~B}$ & 4231.8 & 6946 & $25 \mathrm{Feb}$ \\
\hline 40 & $20021 B$ & 4231.8 & 6946 & $25 \mathrm{Feb}$ \\
\hline 40 & $20022 N$ & 4231.8 & 6946 & $25 \mathrm{Feb}$ \\
\hline 41 & $20027 B$ & 4211 & 7007 & 27 Feb \\
\hline 41 & $20032 \mathrm{~N}$ & 4217 & 7007 & 27 Feb \\
\hline 41 & $20033 B$ & 4211 & 7007 & 27 Feb \\
\hline 41 & $20034 \mathrm{~N}$ & 4211 & 7007 & $27 \mathrm{Feb}$ \\
\hline 47 & $20036 \mathrm{~N}$ & 4211 & 7007 & 27 Feb \\
\hline 42 & & 4152.7 & 6939.5 & 27 Feb \\
\hline 43 & $20051 \mathrm{~B}$ & 4152.7 & 6900.8 & $28 \mathrm{Feb}$ \\
\hline Wh & $20067 \mathrm{~B}$ & 4753 & 6821 & $28 \mathrm{Feb}$ \\
\hline 45 & & 4151.5 & 6738 & 29 Feb \\
\hline 46 & $20093 B$ & 4746.5 & 6650 & $29 \mathrm{Feb}$ \\
\hline 46 & $20094 \mathrm{~N}$ & 4746.5 & 6650 & $29 \mathrm{Feb}$ \\
\hline 47 & & 4142 & 6619.5 & 29 Feb \\
\hline 48 & & 4736 & 6600 & $29 \mathrm{Feb}$ \\
\hline 49 & & 4720 & 6608 & $29 \mathrm{Feb}$ \\
\hline 50 & & 4713.8 & 6657 & 29 Feb \\
\hline 51 & & 4115.4 & 6756 & $1 \mathrm{Mar}$ \\
\hline 52 & $20165 \mathrm{~B}$ & 4113.5 & 6859.7 & $1 \mathrm{Mar}$ \\
\hline 53 & & 4046 & 6859 & $1 \mathrm{Mar}$ \\
\hline 54 & & 4047 & 681 & 1 Mar \\
\hline 55 & & 4048.5 & $67 \quad 43.7$ & 1 Mar \\
\hline 56 & & 4028.8 & 6755.4 & 1 Mar \\
\hline 57 & & 4027.5 & 6859 & $1 \mathrm{Mar}$ \\
\hline 58 & & 4035.5 & 6939 & 2 Mar \\
\hline
\end{tabular}

Recovered

Lat Iong Date

$4116 \quad 70$ I4 $28 \mathrm{JvI} 56$ No returns

No returns

$4528 \quad 6450 \quad 23$ Jun 56 $4204.8 \quad 7013.3 \quad 6$ Oct 56 No returns

No returns

No returns

No returns

No returns

No returns

No returns

No returns

$4126 \quad 7042 \quad 7$ JuI 56

LI $29.7 \quad 7108 \quad 2$ JuI 56

$\begin{array}{llllll}23 & 23 & 70 & 30.3 & 28 & \text { May } 56\end{array}$

$4128.6 \quad 7046.7 \quad 2$ JuI 56

$41 \quad 17.8 \quad 7006 \quad 17$ Jun 56

$4518 \quad 6447 \quad 14$ Oct 56

$4123.1 \quad 70 \quad 30 \quad 16$ Jun 56

47 W 6958 21 Mar 56

$4120.8 \quad 70 \quad 30 \quad 24$ May 56

$47 \begin{array}{llll}40.4 & 71 & 04 & 22 \\ & \text { Jun } & 56\end{array}$

42 04.4. 70 14.2 2 Mar 56

$4137.6 \quad 71 \quad 12 \quad 12 \mathrm{Jul} 56$

$4204.170 \quad 14.6 \quad 8$ Mar 56

4144.97025 .722 Apr 56 No returns

$4134.7 \quad 70 \quad 56.2 \quad 20$ Jun 56 $4136.57016 \quad 15 \mathrm{JuI} 56$ No returns

$4120.5 \quad 7042.5 \quad 6 . J u n 56$ $4121 \quad 7047 \quad 20$ oct 56

No returns

No returns

No returns

No returns

No returns

$4130 \quad 7102.6 \quad 10 \mathrm{JuI} 56$

No $r$ eturns

No returns

No returns

No returis

No returns

No returns 
Released

\begin{tabular}{|c|c|c|c|c|}
\hline Station & Bottle & Lat & Iong & Date \\
\hline 59 & & 4044.7 & $70 \quad 19.4$ & $2 \operatorname{Mar}$ \\
\hline 60 & $20257 \mathrm{~B}$ & 4117 & 7052.7 & 2 Mar \\
\hline 60 & $20260 \mathrm{~N}$ & 4117 & $70 \quad 52.7$ & 2 Mar \\
\hline 60 & $20264 \mathrm{~N}$ & 4117 & $70 \quad 52.7$ & $2 \mathrm{Mar}$ \\
\hline 60 & $20265 B$ & 4117 & 7052.7 & $2 \mathrm{Mar}$ \\
\hline 60 & $20267 \mathrm{~B}$ & 4117 & $70 \quad 52.7$ & 2 Mar \\
\hline
\end{tabular}

Recovered

Iat Long Date No returns

$4119.2 \quad 7048.7 \quad 18$ Apr 56 $\begin{array}{lll}4021 & 7046.5 & 18 \\ 4 a r & 56\end{array}$ $4125.57044 \quad 15 \mathrm{Mar} 56$ $4725.5 \quad 70 \quad 55.5 \quad 26$ Sep 56 $\begin{array}{lll}4126 & 70 \quad 50.9 & 11 \text { Jun } 56\end{array}$ 
ALBATROSS III Cruise 72 21-31 March, 1956

\begin{tabular}{|c|c|c|c|c|c|c|c|}
\hline \multirow[b]{2}{*}{ Station } & \multirow[b]{2}{*}{ Bottle } & \multicolumn{2}{|c|}{ Released } & \multirow[b]{2}{*}{ Date } & \multicolumn{2}{|c|}{ Recovered } & \multirow[b]{2}{*}{ Date } \\
\hline & & Lat & Long & & Lat & Long & \\
\hline 1 & $20272 N$ & 4]. 77.8 & 7100 & 21 Mar & 4058.4 & 7206.6 & 17 Apr 56 \\
\hline 1 & $20273 B$ & 4117.8 & 7100 & $21 \mathrm{Mar}$ & 4130.5 & 7104 & 24 Jun 56 \\
\hline 1 & $20274 \mathrm{~N}$ & 4117.8 & 7100 & 21 Mar & 4116 & 7012.3 & 2 Jun 56 \\
\hline 1 & $20276 \mathrm{~N}$ & 4717.8 & 7100 & $21 \mathrm{Mar}$ & 4058.5 & 7206.2 & 17 Apr 56 \\
\hline 1 & $20278 \mathrm{~N}$ & 4117.8 & 7100 & 21 Mar & 4058.3 & 7207.3 & 17 Apr 56 \\
\hline 2 & & 4038 & $\begin{array}{ll}71 & 01\end{array}$ & 21 Mar & No $r$ & etums & \\
\hline 3 & & 4002.5 & 7059 & 21 Mar & $\mathrm{N}_{\mathrm{O}} \mathrm{r}$ & eturns & \\
\hline 4 & & 4001 & 7013.5 & 22 Mar & No $r$ & eturns & \\
\hline 5 & & 4001 & 6926 & 22 Mar & No $r$ & eturns & \\
\hline 6 & & 395.5 & 6838 & 22 Mar & No $r$ & eturns & \\
\hline 7 & & 3957.5 & 6804.3 & 22 Mar & No $r$ & eturns & \\
\hline 8 & & 4032 & 6743 & 22 Mar & No $r$ & aturns & \\
\hline 9 & $20413 B$ & 4106 & 6726.5 & 22 Mar & 4127.5 & 7046.7 & 7 JuI 56 \\
\hline 9 & $20422 \mathrm{~N}$ & 4706 & 6726.5 & $22 \mathrm{Mar}$ & $4 I 23.4$ & 7030.5 & 26 May 56 \\
\hline 10 & & 4139 & 6708 & $23 \mathrm{Mar}$ & No $r$ & eturns & \\
\hline 17 & & 4209 & 6654 & $23 \mathrm{Mar}$ & No $r$ & eturns & \\
\hline 12 & $20456 \mathrm{~N}$ & 4240 & 6622 & 23 Mar & 4454.5 & 6521 & 25 May 56 \\
\hline 12 & $20460 \mathrm{~N}$ & 4240 & 6622 & 23 Mar & 4509 & 6448 & 21 Jun 56 \\
\hline 13 & $20467 B$ & 4310 & 6613 & $23 \mathrm{Mar}$ & 4450 & 6656 & $14 \mathrm{Jul} 56$ \\
\hline 14 & & 4303.8 & 6531.3 & $23 \mathrm{Mar}$ & No $r$ & eturns & \\
\hline 15 & & 4259 & 6440 & 23 Mar & No $n$ & sturns & \\
\hline 16 & & 4235.5 & 6438.5 & 23 Mar & No $r$ & aturns & \\
\hline 17 & $20511 B$ & 4238.5 & 6543 & 24 Mar & 4452.7 & 6657.8 & 26 Sep 56 \\
\hline 18 & & 4250 & 6723 & 24 Mar & No $n$ & eturns & \\
\hline 19 & & 4248 & 6805 & 24 Mar & No $r$ & eturns & \\
\hline 20 & $20556 \mathrm{~N}$ & 4247 & 6854.5 & $24 \mathrm{Mar}$ & 4522 & 6420 & 7 Sep 56 \\
\hline 21 & $20563 B$ & 4239.7 & 7002 & 24 Mar & 4139 & 7011.5 & 2 Sep 56 \\
\hline 22 & $20579 B$ & 4235 & 7023.5 & 26 Mar & 4115.2 & 7010 & 12 JuI 56 \\
\hline 23 & & 4204.5 & 6952 & 26 Mar & No $r$ & eturns & \\
\hline 24 & & 4204.8 & 6857 & $26 \mathrm{Mar}$ & No $r$ & eturns & \\
\hline 25 & & 4207.5 & 6738 & $26 \mathrm{Mar}$ & No $r$ & eturns & \\
\hline 26 & & 4204.3 & 6700 & $27 \mathrm{Mar}$ & No $r$ & eturns & \\
\hline 27 & & 4201.5 & 6604 & 27 Mar & No $r$ & eturns & \\
\hline $27 \mathrm{~A}$ & $20648 \mathrm{~N}$ & 4203 & 6556 & 27 Mar & 4119 & 7017 & 24 Jul 56 \\
\hline 28 & & 4207 & 6506 & 27 Mar & No $r$ & eturns & \\
\hline 29 & & 4246.5 & 6507 & 27 Mar & No $r$ & eturns & \\
\hline 30 & $20677 \mathrm{~B}$ & 4240.5 & 6608.3 & 28 Mar & 4413 & 6609 & 23 Oct 56 \\
\hline 30 & $20680 \mathrm{~N}$ & 4240.5 & 6608.3 & 28 Mar & 4434.7 & 6556.5 & 7 Jul 56 \\
\hline 31 & & 4217.5 & 6602 & $28 \mathrm{Mar}$ & No $r$ & eturns & \\
\hline 32 & $20710 \mathrm{~N}$ & 47.45 .5 & 6553 & $28 \mathrm{Mar}$ & $4123 . ?$ & 7003 & 25 Nov 56 \\
\hline 33 & & 4150.5 & 6702.5 & 28 Mar & Nc: & eturns & \\
\hline 34 & $20728 \mathrm{~N}$ & 4745.5 & 6800 & 28 Mar & 4122.8 & 7030.3 & I4 JuI 56 \\
\hline $\begin{array}{l}35 \\
36\end{array}$ & & 4147 & 6901.5 & $28 \mathrm{Mar}$ & Nore & turns & \\
\hline 36 & & 4147 & 6946 & 29 Mar & No $r$ & eturns & \\
\hline
\end{tabular}


Released

\begin{tabular}{|c|c|c|c|c|}
\hline Station & Bottle & Lat & Long & Date \\
\hline 37 & & 4730 & 6930 & 29 Mar \\
\hline 38 & $20780 \mathrm{~N}$ & 4725 & 6844 & 29 Mar \\
\hline 39 & & 4730 & 6748.5 & 29 Mar \\
\hline 40 & & 4724.5 & 6627 & 29 Mar \\
\hline 41 & & L1 20 & 6605 & 29 Mar \\
\hline 42 & & 4106 & $66 \quad 27$ & 29 Mar \\
\hline 43 & & 4105 & 6717 & 29 Mar \\
\hline 44 & & 4045.8 & 6713 & $30 \mathrm{Mar}$ \\
\hline 45 & $20866 \mathrm{~N}$ & 4045 & 6644 & 30 Mar \\
\hline 46 & & 4026 & 6718 & 30 Mar \\
\hline 47 & $20892 \mathrm{~N}$ & 4704.3 & 6807.5 & 30 Mar \\
\hline 48 & $20893 B$ & 4027.5 & 6859 & $30 \mathrm{Mar}$ \\
\hline 48 & $20896 \mathrm{~N}$ & 4027.5 & 6859 & 30 Mar \\
\hline 48 & $20899 B$ & 4027.5 & 6859 & 30 Mar \\
\hline 48 & $20903 B$ & 4027.5 & 6859 & 30 Mar \\
\hline 49 & $20907 \mathrm{~B}$ & 4055 & 6937.5 & $30 \mathrm{Mar}$ \\
\hline 49 & $20909 B$ & 4055 & 6937.5 & 30 Mar \\
\hline 49 & $20911 B$ & 4055 & 6937.5 & $30 \mathrm{Mar}$ \\
\hline 49 & 209158 & 4055 & 6937.5 & 30 Mar \\
\hline 50 & $20917 \mathrm{~B}$ & 4027 & 7014 & 31 Mar \\
\hline 50 & $20918 \mathrm{~N}$ & 4027 & $70 \mathrm{IL}$ & $31 \mathrm{Ma}$ \\
\hline 50 & $20921 B$ & 4027 & $70 \quad 14$ & $31 \mathrm{Ma}$ \\
\hline 50 & 20922N & 4027 & $70 \mathrm{I4}$ & $31 \mathrm{Ma}$ \\
\hline 50 & $20927 \mathrm{~B}$ & $40 ? 7$ & 7014 & $31 \mathrm{Ma}$ \\
\hline 50 & $20928 \mathrm{~N}$ & 4027 & $70 \mathrm{I}_{4}$ & $31 \mathrm{Ma}$ \\
\hline 占 & $20930 \mathrm{~N}$ & 4056.8 & $70 \overline{39}$ & $31 \mathrm{Ma}$ \\
\hline 51 & $20934 N$ & 4056.8 & 7039 & $31 \mathrm{Ma}$ \\
\hline 51 & $20936 \mathrm{~N}$ & 4056.8 & 7039 & $32 \mathrm{Ma}$ \\
\hline 52 & $20941 B$ & 4117.8 & 7100 & $31 \mathrm{Ma}$ \\
\hline 52 & $20942 \mathrm{~N}$ & 4117.8 & 7100 & $31 \mathrm{Ma}$ \\
\hline 52 & $20944 \mathrm{~N}$ & 4117.8 & 7100 & $31 \mathrm{Ma}$ \\
\hline 52 & $20946 \mathrm{~N}$ & LI 17.8 & 7100 & 31 Mar \\
\hline 52 & $20948 \mathrm{~N}$ & 4117.8 & 7100 & 31 Mar \\
\hline 52 & $20949 B$ & 4117.8 & 7100 & $31 \mathrm{Ma}$ \\
\hline 52 & $20950 \mathrm{~N}$ & 4117.8 & 7100 & $31 \mathrm{Ma}$ \\
\hline 52 & $20952 \mathrm{~N}$ & 4117.8 & 7100 & $31 \mathrm{Ma}$ \\
\hline
\end{tabular}

Recovered

Iat Lons Date

No returns

$4147 \quad 7002 \quad 12$ Nov 56

No retums

No returns

No returns

No returns

No returns

No returns

$4744.9 \quad 7035.4 \quad 4$ Oct 56 No returns

$4720.7 \quad 7040 \quad 26$ Jun 56

$4723.2 \quad 70 \quad 30.9 \quad 5$ Jun 56

$4117.7 \quad 7008.82$ Jun 56

$41 \quad 29.3 \quad 71 \quad 17.5 \quad 25$ Jun 56

$4723.5 \quad 7003 \quad 8 \mathrm{JuI} 56$

$4738.1 \quad 7017 \quad 30$ May 56

$\begin{array}{llllll}41 & 30 & 71 & 02.6 & 10 & \mathrm{JuI} \\ 56\end{array}$

$\begin{array}{llllll}41 & 27.5 & 70 & 46.5 & 7 & \text { JuI } 56\end{array}$

$4130.5 \quad 7040.9 \quad 4 \mathrm{Jun} 56$

$4130.5 \quad 7105.5 \quad 25$ Jun 56

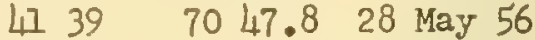

$4129.7 \quad 7108 \quad 24$ Jun 56

$4119 \quad 70 \quad 48.5 \quad 15$ May 56

$47 \begin{array}{llllll}38.3 & 70 & 39 & 7 & \mathrm{JuI} & 56\end{array}$

$4720.8 \quad 70 \quad 30 \quad 25$ May 56

$4720.8 \quad 7030 \quad 22$ Jun 56

$4052.8 \quad 7207 \quad 16$ Apr 56

$4701.3 \quad 7158.3 \quad 5$ Apr 56

$4727.4 \quad 71 \quad 17.6 \quad 24$ Jun 56

$4058.2 \quad 7207 \quad 17$ Apr 56

$\begin{array}{llllll}40 & 58.4 & 72 & 06.6 & 17 & \text { Apr } 56\end{array}$

4051.17226 .421 Apr 56

$4058.4 \quad 7206.6 \quad 17$ Apr 56

$\begin{array}{lllllll}41 & 14.6 & 70 & 07.7 & 7 & \text { Jun } 56\end{array}$

LI $30.5 \quad 7105.3 \quad 17$ Apr 56

4102.37155 .122 Apr 56 
Released

\begin{tabular}{|c|c|c|c|c|}
\hline Station & Bottle & Lat & Long & Date \\
\hline 1 & $20956 \mathrm{~N}$ & 4056 & 7100 & $17 t$ \\
\hline 1 & $20957 \mathrm{~B}$ & 4056 & 7100 & 17 \\
\hline 1 & $20958 \mathrm{~N}$ & 4056 & 7100 & 17 \\
\hline 1 & $20961 \mathrm{~B}$ & 4056 & 7100 & 17 \\
\hline 1 & $20964 \mathrm{~N}$ & 4056 & 7100 & 17 \\
\hline 2 & $20968 \mathrm{~N}$ & 4031.4 & 7100.2 & 17 \\
\hline 3 & & 4000.5 & 7056 & 17 \\
\hline 4 & $20992 \mathrm{~N}$ & 4001.5 & 6958.3 & $18 \mathrm{~A}$ \\
\hline 5 & & 4000.5 & 6852.5 & 18 \\
\hline 6 & & 4000 & 6800 & 18 \\
\hline 8 & & 4701.8 & 6731.8 & 18 \\
\hline 9 & & 4729 & 6711.7 & $18 \mathrm{~A}$ \\
\hline 11 & $21054 \mathrm{~N}$ & 4240 & 6638 & $19 \mathrm{~A}$ \\
\hline 11 & $21060 \mathrm{~N}$ & 4240 & 6638 & 19 \\
\hline 12 & $21065 \mathrm{~B}$ & 4305 & 6628 & $19 \mathrm{~A}$ \\
\hline 12 & $21066 \mathrm{~N}$ & 4305 & 6628 & $19 \mathrm{~A}$ \\
\hline 12 & $21070 \mathrm{~N}$ & 4305 & 6628 & $19 \mathrm{~A}$ \\
\hline 12 & $21072 \mathrm{~N}$ & 4305 & 6628 & 19 \\
\hline 13 & $21075 B$ & 4322.2 & 6620.4 & 19 \\
\hline 13 & $21076 \mathrm{~N}$ & 4322.2 & 6620.4 & 19 \\
\hline 13 & $21078 \mathrm{~N}$ & 4322.2 & 6620.4 & $19 \mathrm{~A}$ \\
\hline 13 & $21079 B$ & 4322.2 & 6620.4 & 19 \\
\hline 13 & $21084 \mathrm{~N}$ & 4322.2 & 6620.4 & 19 \\
\hline $13 A$ & $21088 \mathrm{~N}$ & 4344 & 6638.5 & $19 \mathrm{~A}$ \\
\hline $13 \mathrm{~A}$ & $21089 B$ & 4344 & 6638.5 & $19 \mathrm{~A}$ \\
\hline $13 A$ & $21091 B$ & 4344 & 6638.5 & $19 \mathrm{~A}$ \\
\hline $13 \mathrm{~A}$ & $21092 \mathrm{~N}$ & 4344 & 6638.5 & $19 \mathrm{~A}$ \\
\hline $13 \mathrm{~A}$ & $21096 \mathrm{~N}$ & $43 \mathrm{LH}_{4}$ & 6638.5 & $19 \mathrm{~A}$ \\
\hline$\amalg_{4}$ & $21100 \mathrm{~N}$ & 4355.5 & 6651 & $19 \mathrm{~A}$ \\
\hline 14 & $21102 \mathrm{~N}$ & 4355.5 & 6651 & $19 \mathrm{~A}$ \\
\hline 14 & $21104 \mathrm{~N}$ & 4355.5 & 6651 & $19 \mathrm{~A}$ \\
\hline 14 & $21108 \mathrm{~N}$ & 4355.5 & 665 & $19 \mathrm{~A}]$ \\
\hline 15 & $21120 \mathrm{~N}$ & 4350 & 6802 & $19 \mathrm{~A}$ \\
\hline 16 & $21123 B$ & 4340.5 & 6900 & $19 \mathrm{~A}$ \\
\hline 16 & $21124 \mathrm{~N}$ & 4340.5 & 6900 & $19 \mathrm{~A}$ \\
\hline 16 & $21128 \mathrm{~N}$ & 4340.5 & 6900 & $19 \mathrm{~A}$ \\
\hline 16 & $21129 B$ & 4340.5 & 6900 & $19 \mathrm{~A}$ \\
\hline 17 & $21136 \mathrm{~N}$ & 4329 & 7002.5 & $20 \mathrm{~A}$ \\
\hline 17 & $21140 \mathrm{~N}$ & 4329 & 7002.5 & $20 \mathrm{~A}]$ \\
\hline 18 & $21153 B$ & 4300.4 & 7030 & $20 \mathrm{~A}$ \\
\hline 19 & $21162 \mathrm{~N}$ & 4258.3 & 7000 & $20 \mathrm{~A}]$ \\
\hline 19 & $21163 B$ & 4258.3 & 7000 & $20 \mathrm{Ar}$ \\
\hline
\end{tabular}

Recovered

Lat Irong Date

$\begin{array}{lllll}41 & 21.8 & 70 & 29.7 & 25 \\ 4 & 25 y & 56\end{array}$

U工 $25 \quad 7027 \quad 20$ JuI 56

$4130.4 \quad 7041.6 \quad 15 \mathrm{JuI} 56$

$4129.2 \quad 71 \quad 15.5 \quad 8$ JuI 56

$4140.27000 .8 \quad 2$ Jun 56

$4722.6 \quad 70 \quad 30.2 \quad 15$ Oct 56 No returns

$4127.470 \quad 49.5 \quad 7$ JuI 56

No returns

No returns

No returns

No returns

$4459 \quad 6658 \quad 27$ Jun 56

$4459 \quad 6658 \quad 24 \operatorname{Jun} 56$

$4456.36644 \quad 10$ Oct 56

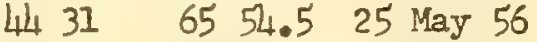

$44156623 \quad 6$ May 56

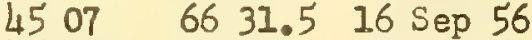

$4500 \quad 6657 \quad 28$ Jul 56

$4434 \quad 6552 \quad 29$ Jul 56

$44416545 \quad 9$ May 56

$44416541 \quad 13 \mathrm{May} 56$

$4435.7 \quad 6558 \quad 8$ May 56

$\begin{array}{llll}4505 & 66 & 39 & 4\end{array}$ Sep 56

$4437 \quad 6543 \quad 9$ May 56

$\begin{array}{lllll}44 & 01 & 68 & 37.5 & 21 \\ \text { JuI } & 56\end{array}$

$4518 \quad 6447 \quad 24 \mathrm{May} 56$

$4520 \quad 6448 \quad 10$ May 56

$\begin{array}{lllll}45 & 02.3 \quad 66 & 53.6 \quad 4 \text { Sep } 56\end{array}$

$4520 \quad 6448 \quad 11$ May 56

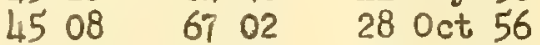

$4447.5 \quad 65 \quad 35 \quad 20$ May 56

$4048.56956 \quad 17$ Nor 56

$\begin{array}{llll}43 & 31 & 70 & 22.4\end{array} 2 \operatorname{Sep} 56$

$\begin{array}{llllll}43 & 10.5 & 70 & 36.5 & 12 & \mathrm{Jun} \\ 56\end{array}$

$\begin{array}{llllll}43 & 38.5 & 70 & 13.7 & 11 & \mathrm{Jun} \\ 56\end{array}$

\begin{tabular}{lllll}
43 & 39 & 70 & 12.8 & 14 \\
\hline
\end{tabular}

$4238 \quad 7036.7 \quad 30$ Sep 56

$4349 \quad 6952.9 \quad 13$ Jun 56

$4449 \quad 6521 \quad 12$ Nov 56

$4515.2 \quad 6548.5 \quad 16$ sep 56

$4510 \quad 6446 \quad 14$ Oct 56 


\begin{tabular}{|c|c|c|c|c|c|c|c|}
\hline Station & Bottle & Lat & Long & Date & $L_{a t}$ & Long & Date \\
\hline 20 & $21171 B$ & 4253.2 & 6855.8 & 20 Apr & 4450 & 6656 & $\Psi_{4} \mathrm{Ju}$ \\
\hline 21 & $21182 \mathrm{~N}$ & 4248.8 & 6803 & 20 Apr & 4455 & 6656 & 16 Jun \\
\hline 21 & $21190 \mathrm{~N}$ & 4248.8 & 6803 & 20 Apr & 4341 & 7007 & $12 \mathrm{Sep}$ \\
\hline 21 & $21192 \mathrm{~N}$ & 4248.8 & 6803 & $20 \mathrm{Apr}$ & 4218 & 7055 & 11 Nov \\
\hline 22 & $21196 \mathrm{~N}$ & 4253 & 6700.5 & 20 Apr & 4528 & 6452 & 26 Sep \\
\hline 22 & $21202 \mathrm{~N}$ & 4253 & 6700.5 & 20 Apr & 4448.5 & 6658.9 & 12 Jun \\
\hline 23 & & 4254 & 6613 & 21 Apr & No & returns & \\
\hline 24 & & 4237 & 6501.5 & $21 \mathrm{Apr}$ & No: & returns & \\
\hline 25 & & 4201 & 6512 & 21 Apr & No & returns & \\
\hline 26 & $212418 B$ & 4201.5 & 6602 & 21 Apr & 4503 & 6504 & 27 Nov \\
\hline 27 & & 4205.4 & 6649 & 21 Apr & 4503 & 6504 & 27 Nov \\
\hline 28 & $21271 B$ & 4158 & 6800 & 22 Apr & 4513 & 6433 & 6 Nov \\
\hline 29 & $21287 \mathrm{~B}$ & 4204.8 & 6903.5 & 22 Apr & 4452 & 6527 & 4 Nov \\
\hline 30 & & $\begin{array}{lll}42 & 06.8\end{array}$ & 6928.5 & 22 Apr & No & returns & \\
\hline 31 & $21302 \mathrm{~N}$ & $42 \mathrm{IL}_{4}$ & 6946.1 & 22 Apr & $42 \mathrm{OL}_{4}$ & 7015 & 27 Oct \\
\hline 31 & $21306 \mathrm{~N}$ & $42 \mathrm{IL}_{4}$ & 6946.1 & 22 Apr & 4327 & 6528 & 7 Sep \\
\hline 32 & & 4233.4 & 7038.8 & 22 Apr & No & returns & \\
\hline 33 & & 4216.5 & 7015.5 & 22 Apr & No & returns & \\
\hline 34 & & 4131.5 & 6940 & $23 \mathrm{Apr}$ & No & retums & \\
\hline 35 & & 4134.5 & 6904 & $23 \mathrm{Apr}$ & No & returns & \\
\hline 36 & $21370 \mathrm{~N}$ & 4128.5 & 6755 & 23 Apr & 4121 & 7050 & $30 \mathrm{Ju}$ \\
\hline 37 & & 4730 & 6654 & 23 Apr & No & returns & \\
\hline 39 & & 4102 & 6627.5 & 24 Apr & No: & returns & \\
\hline 40 & & 4101.5 & 6658.5 & 24 Apr & No & returns & \\
\hline 41 & & 4058.2 & 6805.5 & $24 \mathrm{Apr}$ & No & returns & \\
\hline 42 & & 4103.5 & 6904 & 24 Apr & No & returns & \\
\hline 43 & & $40 \quad 30.3$ & 6851 & 25 Apr & No & returns & \\
\hline 44 & & 4030.5 & 6800 & 25 Apr & No & returns & \\
\hline 45 & & 4034 & 6659 & 24 Apr & No & returns & \\
\hline 46 & & 4113 & 6628.5 & $25 \mathrm{Apr}$ & No & returns & \\
\hline 47 & & 4132 & 6615.5 & 25 Apr & No: & returns & \\
\hline 48 & & 4213.2 & 6546.8 & 25 Apr & No: & returns & \\
\hline 49 & & 4223 & 6532.5 & 26 Apr & No & returns & \\
\hline 50 & & 4259.5 & 6510.1 & 26 Apr & No: & returns & \\
\hline II & & 4244.5 & 6547 & 26 Apr & No & returns & \\
\hline 52 & $21544 \mathrm{~N}$ & 4233 & 6627 & 26 Apr & 4503.5 & $67 \mathrm{OL}$ & 5 Aug \\
\hline 52 & $21548 \mathrm{~N}$ & 4233 & 6627 & 26 Apr & 4431.8 & 6738.4 & $18 \mathrm{JuI}$ \\
\hline 52 & 215518 & 4233 & 6627 & 26 Apr & 4437 & 6543 & $16 \mathrm{Nov}$ \\
\hline 53 & $21554 \mathrm{~N}$ & 4219.8 & 6656.7 & 26 Apr & 4222 & 7058 & 27 Oct \\
\hline 54 & & 4223 & 6602 & 26 Apr & No & returns & \\
\hline 55 & $21578 \mathrm{~N}$ & 4218.6 & 6530.6 & 26 Apr & 4335 & 6526 & $30 \mathrm{JuI}$ \\
\hline 55 & $21581 B$ & 4218.6 & 6530.6 & 26 Apr & 4151 & 6957 & $280 \mathrm{ct}$ \\
\hline 56 & $21595 \mathrm{~B}$ & 4146.3 & 6530.4 & 27 Apr & $?$ & $?$ & 23 Jun \\
\hline 57 & & 4747.5 & 6558 & 27 Apr & No & returns & \\
\hline 58 & & 4745 & 6703.5 & 27 Apr & No & returns & \\
\hline 59 & & 4126.8 & 6653.9 & 27 Apr & No: & returns & \\
\hline 60 & & 4710.5 & 6643.5 & 27 Apr & $\mathrm{N}_{0}$ & returns & \\
\hline 61 & & 4044.5 & 6629.5 & 27 Apr & No : & returns & \\
\hline
\end{tabular}


Released

\begin{tabular}{|c|c|c|c|c|}
\hline Station & Bottre & Iat & Long & Date \\
\hline 62 & $21663 B$ & 4046 & 6739 & 27 Apr \\
\hline 63 & & 4044.5 & 6805.8 & 28 Apr \\
\hline 64 & & 4031.5 & 6900 & 28 Apr \\
\hline 65 & $21701 B$ & 4048 & 7002 & 28 Apr \\
\hline 65 & $21705 \mathrm{~B}$ & 4048 & 7002 & 28 Apr \\
\hline 65 & $21707 \mathrm{~B}$ & 4048 & 7002 & $28 \mathrm{Apr}$ \\
\hline 66 & $21710 \mathrm{~N}$ & 4104.4 & 7034.7 & 28 Apr \\
\hline 66 & $21711 B$ & 4104.4 & 7034.7 & 28 Apr \\
\hline 66 & 21713B & 4104.4 & 7034.7 & $28 \mathrm{Apr}$ \\
\hline 66 & $21714 \mathrm{~N}$ & 4704.4 & $70 \quad 34.7$ & 28 Apr \\
\hline 66 & $21717 \mathrm{~B}$ & 4104.4 & 7034.7 & 28 Apr \\
\hline 66 & $21720 \mathrm{~N}$ & 4104.4 & 7034.7 & 28 Apr \\
\hline 67 & $21721 B$ & 4111 & 7050 & 28 Apr \\
\hline 67 & $21723 B$ & 4111 & 7050 & $28 \mathrm{Apr}$ \\
\hline 67 & $21727 \mathrm{~B}$ & 4111 & 7050 & $28 \mathrm{Apr}$ \\
\hline 67 & $21728 \mathrm{~N}$ & 4111 & 7050 & 28 Apr \\
\hline 67 & $21731 B$ & 4111 & 7050 & $28 \mathrm{Apr}$ \\
\hline 67 & $21723 \mathrm{~N}$ & 411 & 7050 & 28 Apr \\
\hline
\end{tabular}

Recovered

Lat Long Date

$4132.5 \quad 7039.6 \quad 17 \mathrm{JuI} 56$ No returns No returns

$4127.5 \quad 7111.6 \quad 13 \mathrm{Jull} 56$ $4111.9 \quad 7135.5 \quad 17 \mathrm{JuI} 56$ $47 \begin{array}{llll}48 & 70 & 16 & \mathrm{JuI} \\ 56\end{array}$ 47 \begin{tabular}{llll}
17.2 & 70 & 14.8 & 2 \\
\hline & Jun 56
\end{tabular} $4139 \quad 7011.8 \quad 4 \mathrm{Jan} 56$ $4136.9 \quad 7026 \quad 13 \mathrm{JuI} 56$ $4120.97035 \quad 4$ Jun 56 41 $19 \quad 7049 \quad 23$ JuI 56 $4120.9 \quad 7033.5 \quad 4$ Jun 56 $4136.57024 \quad 1$ Jun 56 $4139.4 \quad 6959 \quad 10$ Jun 56 $4138 \quad 7015 \quad 29$ Jun 56 $\begin{array}{lllll}41 & 29.8 & 71 & 02.4 & 26 \\ \mathrm{May} & 56\end{array}$ $4136.57039 \quad 2$ Jun 56 $4139.27007 \quad 23 \mathrm{May} 56$ 
Released

\begin{tabular}{|c|c|c|c|c|}
\hline Station & Bottle & Lat & Iong & Date \\
\hline 1 & $21733 B$ & 4101.7 & $70 \quad 57.7$ & $16 \mathrm{May}$ \\
\hline 1 & $21734 \mathrm{~N}$ & 4101.7 & 7057.7 & $16 \mathrm{May}$ \\
\hline 1 & $21735 \mathrm{~B}$ & $\begin{array}{lll}47 & 01.7\end{array}$ & 7057.7 & $16 \mathrm{May}$ \\
\hline 1 & $21739 B$ & $\begin{array}{ll}47 & 01.7\end{array}$ & 7057.7 & 16 Мay \\
\hline 1 & $21740 \mathrm{~N}$ & $\begin{array}{ll}41 & 01.7\end{array}$ & 7057.7 & $16 \mathrm{May}$ \\
\hline 1 & $21741 \mathrm{~B}$ & 4101.7 & 7057.7 & 16 Мay \\
\hline 1 & $21743 B$ & 4101.7 & 7057.7 & $16 \mathrm{May}$ \\
\hline 1 & $21744 \mathrm{~N}$ & 4101.7 & 7057.7 & $16 \mathrm{May}$ \\
\hline 2 & $21750 \mathrm{~N}$ & 4009 & 7058.6 & $16 \mathrm{May}$ \\
\hline 2 & $21754 \mathrm{~N}$ & 4009 & 7058.6 & $16 \mathrm{May}$ \\
\hline 3 & & 4000.5 & 7100 & $16 \mathrm{May}$ \\
\hline 4 & & 3957.8 & 6933 & 17 May \\
\hline 5 & $21782 \mathrm{~N}$ & 4000.5 & 6851 & 17 May \\
\hline 5 & $21785 \mathrm{~B}$ & 4000.5 & 6851 & 17 May \\
\hline 5 & $21789 \mathrm{~B}$ & 4000.5 & 6851 & 17 May \\
\hline 6 & & 4001.5 & 6759.8 & 17 May \\
\hline 7 & & 4039 & 6737.5 & 17 May \\
\hline 8 & $21818 \mathrm{~N}$ & 4703 & 6717 & 17 May \\
\hline 8 & $21819 \mathrm{~B}$ & 4103 & 6717 & 17 May \\
\hline 9 & & 4127 & 6651 & 17 May \\
\hline 10 & $21844 \mathrm{~N}$ & 4157 & 6630 & $18 \mathrm{May}$ \\
\hline 17 & $21854 \mathrm{~N}$ & 4234 & 6606 & $18 \mathrm{May}$ \\
\hline 11 & $21855 \mathrm{~B}$ & 4234 & 6606 & $18 \mathrm{Mzy}$ \\
\hline 11 & $21859 \mathrm{~B}$ & 4234 & 6606 & $18 \mathrm{May}$ \\
\hline 12 & $21872 \mathrm{~N}$ & 4301.5 & 6546 & $18 \mathrm{May}$ \\
\hline 13 & $21882 \mathrm{~N}$ & 4315.4 & $\begin{array}{lll}66 & 03.7\end{array}$ & 18 May \\
\hline 13 & 218858 & 4315.4 & $\begin{array}{lll}66 & 03.7\end{array}$ & 18 May \\
\hline 13 & $21887 \mathrm{~B}$ & 4315.4 & $\begin{array}{lll}66 & 03.7\end{array}$ & 18 May \\
\hline 13 & $21888 \mathrm{~N}$ & 4315.4 & $\begin{array}{lll}66 & 03.7\end{array}$ & $18 \mathrm{May}$ \\
\hline I4 & $21889 \mathrm{~B}$ & 4317 & 6643 & 18 May \\
\hline 14 & $21896 \mathrm{~N}$ & 4317 & 6643 & 18 May \\
\hline 14 & $21898 \mathrm{~N}$ & 4317 & 6643 & 18 May \\
\hline 15 & $21904 \pi$ & 4316.5 & 6723 & $18 \mathrm{May}$ \\
\hline 16 & & 4345.2 & 6720.9 & $18 \mathrm{May}$ \\
\hline 17 & $21926 \mathrm{~N}$ & 4344.3 & 6625.7 & 19 May \\
\hline 17 & $21931 B$ & 4344.3 & 6625.7 & 19 May \\
\hline 18 & $219411 B$ & 4400.2 & 6626.2 & 19 May \\
\hline 18 & $21984 \mathrm{~N}$ & 4400.2 & 6626.2 & 19 May \\
\hline 19 & $21954 \mathrm{~N}$ & $44 \quad 15.5$ & 6655 & 19 May \\
\hline 19 & $27958 \mathrm{~N}$ & 4475.5 & 6655 & 19 May \\
\hline 20 & & 4421 & 6721.8 & I9 May \\
\hline 21 & $21980 \mathrm{~N}$ & 4428 & 6637 & 19 :ay \\
\hline
\end{tabular}

Recovered

Iat Long Date

$4730.5 \quad 7104 \quad 24 \mathrm{Jum} 56$ $4716.5 \quad 7013.4 .2$ Jun 56

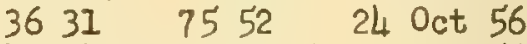
$4124.2 \quad 70 \quad 31.5 \quad 10$ Jun 56 $\begin{array}{lllll}41 & 17.2 & 70 & 14.8 & 4 \\ \mathrm{Jun} & 56\end{array}$ $4123.17030 \quad 9$ Jun 56 $4123.2 \quad 70 \quad 30.8 \quad 5$ Jun 56 $4139.4 \quad 7007 \quad 21$ Jun 56 $\begin{array}{llll}3541 & 7529 & 7 \text { Nov } 56\end{array}$ $4120.8 \quad 70 \quad 50.4 \quad 12$ Jun 56 No returns No returns

$4109 \quad 7134 \quad 1$ sep 56 $4121 \quad 7029 \quad 30 \mathrm{JuI} 56$ $\begin{array}{lll}3546 & 7531 & 1 \text { Nov } 56\end{array}$ No returns No returns

$4121.87030 \quad 14 \mathrm{JuI} 56$ $\begin{array}{llllll}41 & 21 & 70 & 01.6 & 21 & \mathrm{Ju} \\ 56\end{array}$ No returns

$\begin{array}{llll}43 & 25 & 6537 & 27 \\ \text { JuI } & 56\end{array}$ 44 $516658 \quad 29 \mathrm{Jul} 56$ $4520 \quad 6447 \quad 29$ Aug 56 $4744.7 \quad 7025 \quad 27$ oct 56 44 $28 \quad 63 \quad 36 \quad 22$ Aug 56 $\begin{array}{llllllll}42 & 02.5 & 70 & 03.7 \quad 30 & \text { Sep } 56\end{array}$ $4202 \quad 7006 \quad 160$ ct 56 $4342 \quad 6950 \quad 1 \mathrm{JuI} 56$ $4342 \quad 6950 \quad 3$ JuI 56 $\begin{array}{lllllll}4 & 58.5 & 67 & 00.7 & 24 & \text { JuI } & 56\end{array}$ $\begin{array}{lllll}43 & 33.4 & 70 & 17 & 19 \\ \text { Jun } & 56\end{array}$

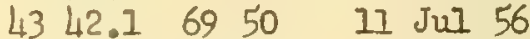
$4527.5 \quad 6452 \quad 2$ Aug 56 No retums

$4427 \quad 6607 \quad 2$ Jun 56 $4430 \quad 6606 \quad 30$ May 56 $4448 \quad 6647 \quad 25 \mathrm{JuI} 56$ $4405 \quad 6611 \quad 24 u_{\text {ct }} 56$

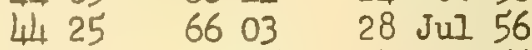
$\begin{array}{llllll}43 & 48.8 & 70 & 05.7 \quad 4 \text { Aug } 56\end{array}$ No returns

44. $24.46800 \quad 23$ Sep 56 


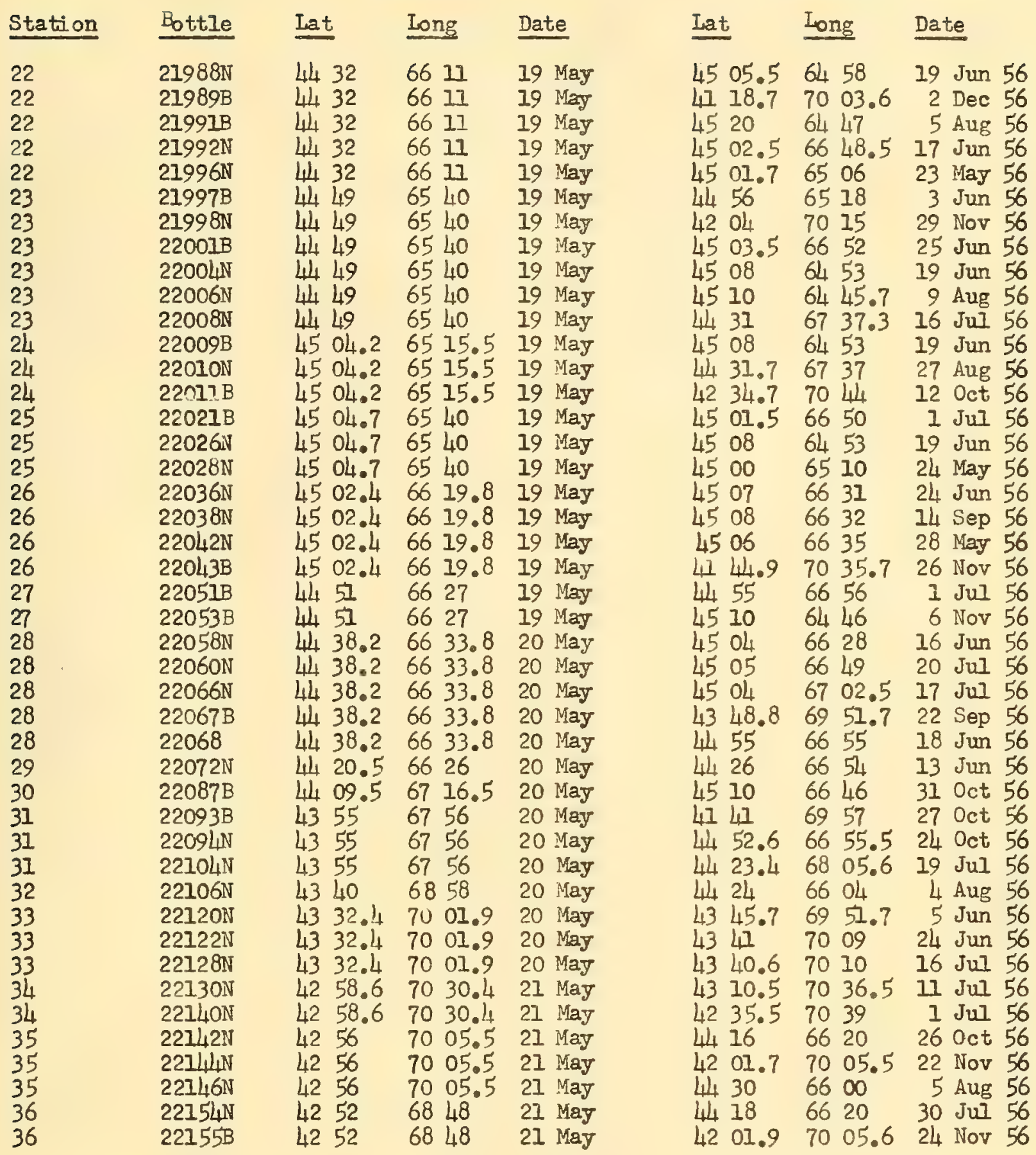




\begin{tabular}{|c|c|c|c|c|c|c|c|}
\hline Station & Bottle & Lat & Long & Date & Iiat & Long & Date \\
\hline 37 & & 4249.8 & 6800.9 & $21 \mathrm{May}$ & No & -eturns & \\
\hline 38 & $22181 B$ & 4245.4 & 6647 & 21 May & 4507 & 6424 & 7 Oct \\
\hline 38 & $22182 \mathrm{~N}$ & 4245.4 & 6647 & 21 May & 41459 & 6657 & 25 Juี \\
\hline 38 & $22184 \mathrm{~N}$ & 4245.4 & 6647 & 21 May & $\begin{array}{ll}42 & 03.7\end{array}$ & 7014.7 & 26 Nov \\
\hline 39 & $22193 B$ & 4242.4 & 6555 & 21 May & 4344.7 & 7000.5 & 16 Sep \\
\hline 40 & & 4235.2 & 6503.5 & 22 May & No & eturns & \\
\hline 41 & & 4259 & 6503.3 & 22 May & No & etums & \\
\hline 42 & $22230 \mathrm{~N}$ & 4302 & 6603 & $22 \mathrm{May}$ & 4345.5 & 6937 & $23 \mathrm{Aug}$ \\
\hline 42 & $22235 B$ & 4302 & 6603 & $22 \mathrm{May}$ & 4118 & 7005 & 23 Nov \\
\hline 43 & $22237 B$ & 4304.3 & $665 ?$ & 22 May & 4149 & 6958 & $30 \mathrm{Aug}$ \\
\hline 43 & $22238 \mathrm{~N}$ & 4304.3 & 6657 & 22 May & 4438 & 6642 & 27 Juา \\
\hline 43 & $22245 B$ & 4304.3 & 6657 & $22 \mathrm{May}$ & 4143.5 & 6955.7 & 30 Sep \\
\hline 44 & & 4220.3 & 6700 & 22 May & No & eturns & \\
\hline 45 & & 4217 & 6548.5 & $22 \mathrm{May}$ & No & eturns & \\
\hline 46 & & $421_{4.5}$ & 6502.7 & 23 May & No & - etums & \\
\hline 47 & & 4759.2 & $6504 . ?$ & 23 Мay & No & eturns & \\
\hline 48 & $22307 B$ & 4205 & 6600 & 23 May & 3622.5 & 7549 & 2 Nov \\
\hline 49 & & $42 \quad 10.4$ & 6702 & 23 May & No & -eturns & \\
\hline 50 & & 4204 & 6811 & 23 May & No & - eturns & \\
\hline 51 & & 4204 & 6858 & 23 May & No & returns & \\
\hline 52 & & 4203.5 & 6932.9 & 24 May & No & returns & \\
\hline 53 & $22366 \mathrm{~N}$ & 4238.2 & 6933 & 24 May & 4503.6 & 6627.5 & 14 Sep \\
\hline 54 & $22369 B$ & 4234.2 & 7034 & 24 May & 4234.5 & 7042.5 & 22 JuI \\
\hline 54 & $22370 \mathrm{~N}$ & 4234.2 & 7034 & 24 Маy & 4235 & 7041.8 & 27 May \\
\hline 54 & $22373 B$ & 4234.2 & 7034 & 24 May & 4235 & 7041.8 & 27 May \\
\hline 54 & $2237 \mathrm{LN}$ & 4234.2 & 7034 & $24 \mathrm{May}$ & 4234.8 & 7044 & $28 \mathrm{May}$ \\
\hline 54 & 223758 & 4234.2 & 7034 & $24 \mathrm{May}$ & 4235 & 7047.8 & 27 May \\
\hline 54 & $22376 \mathrm{~N}$ & $4234 . ?$ & 7034 & 24 May & $4234 . ?$ & 7044.2 & 26 May \\
\hline 54 & $22380 \mathrm{~N}$ & 4234.2 & 7034 & 24 May & 4235 & 7041.8 & 28 May \\
\hline 55 & & 4209.7 & 7031.2 & $24 \mathrm{May}$ & No & ceturns & \\
\hline 56 & & 4206.3 & 6957.5 & $24 \mathrm{May}$ & No & -eturns & \\
\hline 57 & & 4131.3 & $6 9 \longdiv { 4 5 }$ & 24 May & No & ceturns & \\
\hline 58 & $22428 \mathrm{~N}$ & 4137.2 & 6900 & $24 \mathrm{May}$ & 3654 & 7559.3 & 28 oct \\
\hline 59 & & 4126 & 6744.5 & $25 \mathrm{May}$ & No & ceturns & \\
\hline 60 & & 4132 & 6657 & 25 May & No & returns & \\
\hline 61 & & 4131.6 & 6618.7 & 25 May & No & ceturns & \\
\hline 62 & & 4118.8 & 6557.7 & 25 May & No & ceturns & \\
\hline 63 & & 4706.5 & 6612 & $25 \mathrm{May}$ & No & -eturns & \\
\hline 64 & $22494 \mathrm{~N}$ & 4106 & 671.5 .5 & 25 May & 4120 & $70 \quad 02$ & $3 \mathrm{Aug}$ \\
\hline 65 & $22506 \mathrm{~N}$ & 4106.5 & 6759.5 & $26 \mathrm{May}$ & 4455 & 6521 & $3 \mathrm{Nov}$ \\
\hline 66 & $22514 N$ & 4059.5 & 6851 & $26 \mathrm{May}$ & 3615 & 7547 & $2 \mathrm{Nov}$ \\
\hline 66 & $22522 \mathrm{~N}$ & 4059.5 & 6851 & 26 May & 3652 & 7559 & 4 Nov \\
\hline 67 & $22533 B$ & 4059 & 6912.8 & $26 \mathrm{May}$ & 3632 & 7552 & $1 \mathrm{Nov}$ \\
\hline 68 & & 4047 & 6908 & 26 May & No & returns & \\
\hline 69 & & 4043.3 & 6845.3 & 26 May & No & ceturns & \\
\hline 70 & & 4040.6 & 6755 & $26 \mathrm{May}$ & No & ceturns & \\
\hline
\end{tabular}




\begin{tabular}{|c|c|c|c|c|}
\hline Station & Bottle & Lat & Iong & Date \\
\hline 71 & & $40 \quad 37.2$ & 6724 & 26 May \\
\hline 72 & & 4038.5 & 6646.6 & 26 May \\
\hline 73 & & 4005 & 6650 & 27 May \\
\hline 74 & & 4023.5 & 6728 & 27 May \\
\hline $74 x$ & $22632 \mathrm{~N}$ & 4037.2 & 6801.3 & 27 May \\
\hline 75 & & 4018 & 6812 & 27 May \\
\hline 76 & $22654 \mathrm{~N}$ & 4013 & 6823 & 27 May \\
\hline 76 & $22656 \mathrm{~N}$ & 4013 & 6823 & 27 Мау \\
\hline 77 & & 4028 & 6859.5 & 27 May \\
\hline 78 & $22673 B$ & 4011 & 6909 & 27 May \\
\hline 78 & $22675 \mathrm{~B}$ & 4011 & 6909 & 27 May \\
\hline 79 & $22684 \pi$ & 4021 & 7021.4 & $28 \mathrm{May}$ \\
\hline 79 & $22685 B$ & 4021 & 7021.4 & $28 \mathrm{May}$ \\
\hline 79 & $22689 B$ & 4021 & 7021.4 & $28 \mathrm{May}$ \\
\hline 79 & $22690 \mathrm{~N}$ & 4021 & 7021.4 & $28 \mathrm{May}$ \\
\hline 79 & $22691 B$ & 4021 & 7021.4 & $28 \mathrm{May}$ \\
\hline 79 & $22692 \mathrm{~N}$ & 4021 & 7021.4 & 28 May \\
\hline 80 & & 4007 & $70 \quad 25.5$ & 28 Mzy \\
\hline 81 & & 3946.5 & 7027.5 & 28 May \\
\hline 82 & & 3950.7 & 7101 & 28 May \\
\hline 83 & & 4012 & 7119 & 28 May \\
\hline 84 & & 4028.7 & 7135.2 & 28 May \\
\hline $85^{\circ}$ & & 4003 & 7200.3 & $28 \mathrm{May}$ \\
\hline 86 & & 4031.5 & $\begin{array}{lll}72 & 02.3\end{array}$ & 29 May \\
\hline 87 & & 4045.5 & 7204 & 29 May \\
\hline 88 & & 4102 & 7132 & 29 Мay \\
\hline 89 & 22801B & 4117.5 & 7100 & 29 Мay \\
\hline 89 & $22803 B$ & 4117.5 & 7100 & 29 Мау \\
\hline 89 & $22804 \mathrm{~N}$ & 47.5 & 7100 & 29 May \\
\hline 89 & $22808 \mathrm{~N}$ & 4117.5 & 7100 & 29 May \\
\hline 89 & $22810 \mathrm{~N}$ & 4117.5 & 7100 & $29 \mathrm{May}$ \\
\hline 89 & $22811 B$ & 4117.5 & 7100 & 29 May \\
\hline
\end{tabular}

Iat Iong Date

No returns

No r eturns

No returns

No returns

$4136 \quad 7039 \quad 24$ JuI 56

No returns

$\begin{array}{llll}3559 & 7538 & 26 & \text { Oct } 56\end{array}$

$4123 \quad 7030 \quad 10$ Aug 56

No returns

$4130 \quad 7102 \quad 21 \mathrm{JuI} 56$

LI I5 $7010 \quad 2 \mathrm{JuI} 56$

$4128.5 \quad 7048 \quad 1$ JuI 56

$47 \begin{array}{llll}15 & 7010 & 3 \mathrm{JuI} & 56\end{array}$

$\begin{array}{lllllll}41 & 32.5 & 70 & 39.5 & 23 & \mathrm{JuI} & 56\end{array}$

$4125.270 \quad 55.6 \quad 26$ Jun 56

$4134 \quad 7031 \quad 11 \mathrm{Jul} 56$

$\begin{array}{llllll}4133 & 70 & 39.2 & 21 & \mathrm{Jul} & 56\end{array}$ No returns

No returns

No returns

No r e turns

No returns

No returns

No returns

No r eturns

No returns

$4118.3 \quad 7047.4 \quad 4 \mathrm{Jun} 56$

$4120 \quad 7049.5 \quad 1$ Jun 56

4I. $39.2 \quad 7045.4 \quad 4.5 u I 56$

$4731.77040 .6 \quad 6$ Jun 56

$4121 \quad 7050.4 \quad 3$ Jun 56

$4718.3 \quad 70 \quad 47.4 \quad 4$ Jun 56 


\section{Released}

\begin{tabular}{|c|c|c|c|c|}
\hline Station & Bottle & Lat & Long & Date \\
\hline 1 & 22821B & 4041 & 7101 & $11 \mathrm{Jun}$ \\
\hline 1 & $2281 / 4 \mathrm{~N}$ & $4 0 \longdiv { 4 1 }$ & 7101 & 11 Jun \\
\hline 2 & & 4001 & 7100 & 11 Jun \\
\hline 3 & & 4000 & 7010 & 12 Jun \\
\hline 4 & & 3958 & 6918 & $12 \mathrm{Jun}$ \\
\hline 5 & & 3958.5 & $68 \lcm{46}$ & 12 Jun \\
\hline 6 & & 4003 & 6800 & 12 Jun \\
\hline 7 & & 4031.5 & 6740.5 & 12 Jun \\
\hline 8 & $22899 B$ & 4105 & 6717 & 12 Jun \\
\hline 9 & & 4136.5 & 6658.5 & 12 Jun \\
\hline 10 & & 4207 & 6631 & 13 Jun \\
\hline 11 & $22936 \mathrm{~N}$ & 4229 & 6606.5 & 13 Jun \\
\hline 11 & $22937 B$ & 4229 & 6606.5 & $13 \mathrm{Jun}$ \\
\hline 12 & $22952 \mathrm{~N}$ & 4300 & 6554 & 13 Jun \\
\hline 12 & $22954 \mathrm{~N}$ & 4300 & 6554 & 13 Jun \\
\hline 13 & $22957 \mathrm{~B}$ & 4312 & 6624 & 13 Jun \\
\hline 13 & $22960 \mathrm{~N}$ & 4312 & 6624 & 13 Jun \\
\hline 14 & & 4311 & 6659 & 13 Jun \\
\hline 15 & & 4333.8 & 6615.3 & 13 Jun \\
\hline 16 & $23000 \mathrm{~N}$ & 4335 & 6703.2 & 13 Jun \\
\hline 17 & $23016 \mathrm{~N}$ & 4402 & 6637.5 & $14 \mathrm{Jun}$ \\
\hline 18 & $23028 \mathrm{~N}$ & W4 17.5 & 6630 & I4 Jun \\
\hline 19 & $23038 \mathrm{~N}$ & 4424 & $6 6 \longdiv { 4 2 . 5 }$ & 14 Jun \\
\hline 20 & $20347 \mathrm{~B}$ & 4444 & 6600 & Ils Jun \\
\hline 20 & $23050 N$ & 4444 & 6600 & 14 Jun \\
\hline 20 & $23051 B$ & 4444 & 6600 & 14 Jun \\
\hline 21 & $23056 \mathrm{~N}$ & 4503.5 & 6518 & If Jun \\
\hline 21 & $23059 \mathrm{~B}$ & 4503.5 & 6518 & 14 Jun \\
\hline 21 & $23060 \mathrm{~N}$ & 4503.5 & 6518 & $14 \mathrm{Jun}$ \\
\hline 21 & $23064 \mathrm{~N}$ & 4503.5 & 6518 & I4 Jun \\
\hline 22 & $23068 \mathrm{~N}$ & 4500.8 & 6607 & $14 \mathrm{Jun}$ \\
\hline 22 & $23070 \mathrm{~N}$ & 4500.8 & 6607 & 14 Jun \\
\hline 22 & 23071B & 4500.8 & 6607 & 14 Jun \\
\hline 23 & $23084 \mathrm{~N}$ & 4458.8 & 6633.8 & $14 \mathrm{Jun}$ \\
\hline 24 & $23096 \mathrm{~N}$ & 4432 & 6612 & $\mathrm{IL}_{4} \mathrm{Jun}$ \\
\hline 24 & $23097 \mathrm{~B}$ & 4432 & 6612 & 14 Jun \\
\hline 25 & & 4413.5 & 6658 & 15 Jun \\
\hline 26 & & 4358 & 6734 & 15 Jun \\
\hline 27 & $23125 \mathrm{~B}$ & 4350 & 6757.5 & 15 Jun \\
\hline 27 & $23126 \mathrm{~N}$ & 4350 & 6737.5 & 15 Jun \\
\hline 28 & $23140 N$ & 4346.5 & 6840 & $15 \mathrm{Jun}$ \\
\hline 28 & $23143 B$ & 4346.5 & 6840 & 15 Jun \\
\hline 28 & $23146 \mathrm{~N}$ & 4346.5 & 6840 & 15 Jun \\
\hline
\end{tabular}

Recovered

Lat Iong Date

$3623 \quad 7549 \quad 23$ oct 56

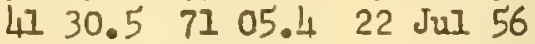
No returns

No returns

No returns

No returns

No returns

No returns

$\begin{array}{llll}3803 \quad 75 & 13.5 \quad 4 \text { Nov } 56\end{array}$ No returns

No returns

$4435 \quad 6552 \quad 6$ Aug 56

$4452 \quad 6527 \quad 13$ Nor 56

$4140 \quad 6956 \quad 19$ Oct 56

$4156.6 \quad 6959 \quad 3$ Nov 56

$\begin{array}{llllll}42 & 01.9 & 70 & 05.6 & 23 & \text { Nov } 56\end{array}$

$\begin{array}{lll}4512 & 6437 \quad 10 \mathrm{Sep} & 56\end{array}$

No returns

No returns

$\begin{array}{llllll}42 & 37.8 & 70 & 39.4 & \boldsymbol{H}_{4} & \text { Oct } 56\end{array}$ $4502.5 \quad 6647 \quad 6$ Sep 56 $4359.1 \quad 6609.6 \quad 25$ Nov 56

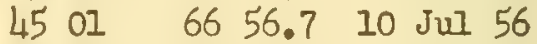
$4439 \quad 6721 \quad 22$ Aug 56 $\begin{array}{lllll}45 & 04.5 & 66 & 28 & 1\end{array}$ $4238.4 \quad 6600 \quad 4$ Nov 56 $\begin{array}{lllll}45 & 02.5 & 66 & 48.5 & 29 \\ \operatorname{Jun} & 56\end{array}$ $4503 \quad 6646 \quad 25$ Oct 56 $4152 \quad 6957 \quad 27$ Oct 56

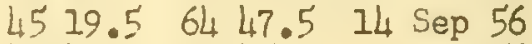
44 $46 \quad 6645 \quad 30 \mathrm{JuI} 56$ $4439.46646 \quad 5$ Aug 56 $4450.3 \quad 66 \quad 57.1 \quad 16 \mathrm{JuI} 56$ $\begin{array}{llllll}40 & 17.7 & 70 & 06.6 & 11 & \text { Nov } 56\end{array}$ $4466 \quad 66$ 44 $29 \mathrm{JuI} 56$ $4507 \quad 6631 \quad 14 \operatorname{sep} 56$ No returns

No returns

4201.97005 .624 Nov 56 44 $52 \quad 6656 \quad 21$ Sep 56 $4502 \quad 6652 \quad 24$ Aug 56 $4351.3 \quad 6952.7 \quad 30$ Sep 56 $43 \quad 45.7 \quad 6951.82$ JuI 56 


\begin{tabular}{|c|c|c|c|c|c|c|}
\hline Station & Bottle & Lat & Iong & Date & Long & Date \\
\hline 28 & $23 \mathrm{IL}_{4} \mathrm{~B}$ & 4346.5 & 6840 & 15 Jun & 4344 & 4 Aug 56 \\
\hline 29 & $23160 \mathrm{~N}$ & 4339 & 6938.2 & 15 Jun & $\begin{array}{llllll}43 & 43.1 & 70 & 08.1\end{array}$ & $14 \mathrm{JuI} 56$ \\
\hline 30 & & 4322.1 & 6917.5 & $15 \mathrm{Jun}$ & No returns & \\
\hline 31 & $23180 \mathrm{~N}$ & 4305.5 & 7005 & $15 \mathrm{Jun}$ & $4508 \quad 6453$ & 9 Aug 56 \\
\hline 31 & $23184 \mathrm{~N}$ & 4305.5 & 7005 & 15 Jun & 4503.2 & 10 Sep 56 \\
\hline 32 & $23196 \mathrm{~N}$ & 4301.5 & 6930.5 & 16 Jun & $4502.5 \quad 66 \quad 53.5$ & 14 Sep 56 \\
\hline 33 & & & 6845.5 & 16 Jun & No returns & \\
\hline 34 & $23209 B$ & 4253 & 6752 & 16 Jun & $414 \quad 6623.3$ & 14 Sep 56 \\
\hline 35 & $23228 \mathrm{~N}$ & 4247.5 & 6719.5 & $16 \mathrm{Jun}$ & 6608 & 3 Aug 56 \\
\hline 36 & $23234 \mathrm{~N}$ & 4245 & 6619 & $16 \mathrm{Jun}$ & 418 & 31 Juา 56 \\
\hline 36 & $23235 B$ & 4245 & 6619 & 16 Jun & $4434 \quad 6552$ & 29 JuI 56 \\
\hline 36 & $23236 \mathrm{~N}$ & 4245 & 6619 & $16 \mathrm{Jun}$ & $43 \lcm{46.7 \quad 6608}$ & 9 JuI 56 \\
\hline 37 & & 4244.8 & 6511.5 & 17 Jun & No returns & \\
\hline 38 & $23258 \mathrm{~N}$ & 4250 & 6545 & 17 Jun & $4340 \quad 6552$ & 12 JuI 56 \\
\hline 38 & $23259 \mathrm{~B}$ & 4250 & 6545 & 17 Jun & 4429.5 & 7 Aug 56 \\
\hline 38 & $23262 \mathrm{~N}$ & 4250 & 6545 & 17 Jun & 4440 & 29 JuI 56 \\
\hline 38 & $23263 B$ & 4250 & 6545 & 17 Jun & 6607.5 & 3 Aug 56 \\
\hline 38 & $23268 \mathrm{~N}$ & 4250 & 6545 & 17 Jun & 4423 & 31 Jง 56 \\
\hline 39 & $23070 \mathrm{~N}$ & 4238 & 6643.5 & $17 \mathrm{Jun}$ & $\begin{array}{llll}42 & 06.8 \quad 70 & 40.3\end{array}$ & 1 Nov 56 \\
\hline 40 & & 4222.8 & 6545.1 & 17 Jun & No returns & \\
\hline 41 & & 4204.8 & 6534.1 & 17 Jun & No returns & \\
\hline 42 & & 4753.5 & 6543.5 & 17 Jun & No returns & \\
\hline 43 & & 4126.1 & 6601 & 17 Jun & No returns & \\
\hline $4 \pi_{1}$ & & 4124.5 & 6645 & 17 Jun & No returns & \\
\hline 45 & $23348 \mathrm{~N}$ & 4728 & 6726 & 18 Jun & $3613 \quad 7546$ & 31 Oct 56 \\
\hline 46 & & 4731 & 6813.3 & 18 Jun & No returns & \\
\hline 47 & $23374 N$ & 4129.5 & 6930 & 18 Jun & $3643 \quad 7556$ & 26 oct 56 \\
\hline 48 & & 4755.5 & 6942.5 & 18 Jun & No r eturns & \\
\hline 49 & $23398 \mathrm{~N}$ & 4218.5 & 7010 & 18 Jun & $\begin{array}{llll}44 & 28.2 & 66 & 08.3\end{array}$ & 9 Oct 56 \\
\hline 50 & $23477 B$ & 4234.5 & 7027 & 18 Jun & $4518 \quad 6432$ & 22 Oct 56 \\
\hline 51 & & 4246 & 7024 & 19 Jun & No returns & \\
\hline 52 & & 4242.3 & 6915.8 & 19 Jun & No returns & \\
\hline 53 & $23448 \mathrm{~N}$ & 4206 & 6910 & 19 Jun & $4420 \quad 6608$ & 4 Aug 56 \\
\hline 54 & & 4205 & 6822 & 19 Jun & No returns & \\
\hline 55 & & 4205 & 6736 & 19 Jun & No returns & \\
\hline 56 & & 4207 & 6651 & 19 Jun & No returns & \\
\hline 57 & $43485 B$ & 4743 & 6608 & 20 Jun & $4756 \quad 6540$ & 9 JuI 56 \\
\hline 58 & & 4728 & 6625 & 20 Jun & No returns & \\
\hline 59 & & 4709 & 6643 & $20 \mathrm{Jun}$ & No returns & \\
\hline 60 & & 4104.5 & 6650 & $20 \mathrm{Jun}$ & No returns & \\
\hline 61 & & 4105 & 6722 & $20 \mathrm{Jun}$ & No returns & \\
\hline 62 & & 4101 & 6816 & 20 Jun & No r eturns & \\
\hline 63 & & 4102.8 & 6900.8 & 20 Jun & No returns & \\
\hline 64 & & 4052 & 6903 & 20 Jun & No retums & \\
\hline 65 & & 4043.5 & 6828 & 20 Jun & No returns & \\
\hline
\end{tabular}




\begin{tabular}{|c|c|c|c|c|c|c|c|}
\hline Station & Bottle & Lat & Long & Date & Iat & Long & Date \\
\hline 66 & & 4045.5 & 6732 & 21. Jun & \multicolumn{3}{|c|}{ No returns } \\
\hline 67 & & 4044 & 6701 & 21 Jun & \multicolumn{3}{|c|}{ No returns } \\
\hline 68 & $23625 B$ & 4025.7 & 6719.3 & 21 Jun & 3600 & 7539 & 190 ct 56 \\
\hline 69 & & 4040 & 6743 & ח 21 תuJ & \multicolumn{3}{|c|}{ No returns } \\
\hline 70 & & 4027.8 & 6756.5 & 21 Jun & \multicolumn{3}{|c|}{ No returns } \\
\hline 71 & & 4013 & 6820 & 21 Jun & \multicolumn{3}{|c|}{ No returns } \\
\hline 72 & & 4037 & 6851 & 21 Jun & \multicolumn{3}{|c|}{ No returns } \\
\hline 73 & $23678 \mathrm{~N}$ & 4019 & 6924 & 21 Jun & 4121 & 7028 & 20 Aug 56 \\
\hline 73 & $23684 \mathrm{~N}$ & 4019 & 6924 & 21 Jun & 3544 & 7529 & $180 \mathrm{ct} 56$ \\
\hline 73 & $23686 \mathrm{~N}$ & 4019 & 6924 & 21 Ju & 4118.5 & 7047.6 & 22 JuI 56 \\
\hline 73 & $23688 \mathrm{~N}$ & 4019 & 6924 & 21 Jun & 4140 & 6959 & $28 \mathrm{JuI} 56$ \\
\hline 74 & & 3953 & 6942.6 & 22 Jun & \multicolumn{3}{|c|}{ No returns } \\
\hline 75 & $23701 B$ & 4016 & 7005 & 22 Jun & 4126.2 & 7041.5 & $11 \mathrm{Aug} 56$ \\
\hline 75 & $23702 N$ & 4016 & 7005 & 22 Jun & 4123 & 7030.5 & 1 Sep 56 \\
\hline 75 & $23704 \mathrm{~N}$ & 4016 & 7005 & 22 Jun & 3623.5 & 7549.4 & 27 oct 56 \\
\hline 75 & 237058 & 4016 & 7005 & 22 Jun & 4130.5 & 7104 & 19 Aug 56 \\
\hline 75 & $23706 \mathrm{~N}$ & 4016 & 7005 & 22 Jun & 4720 & 7049.5 & 17 Aug 56 \\
\hline 75 & $23707 \mathrm{~B}$ & 4016 & 7005 & 22 Jun & 3552 & 7534 & 11 Nov 56 \\
\hline 75 & $23708 \mathrm{~N}$ & 4016 & 7005 & 22 Jun & 4123 & 7002 & $29 \mathrm{JuI} 56$ \\
\hline 75 & $23712 \mathrm{~N}$ & 4016 & 7005 & 22 Jun & 4177.1 & 7014.9 & 22 JuI 56 \\
\hline 76 & $23714 N$ & 4038 & 7023.2 & 22 Jun & 4.125 .4 & 7055 & 9 JuI 56 \\
\hline 76 & $23715 B$ & 4038 & 7023.2 & 22 Jun & 4125.4 & 7054 & 18 Jul 56 \\
\hline 76 & $23717 B$ & 4038 & 7023.2 & 22 Jun & 4139 & 7010 & 22 JuI 56 \\
\hline 76 & $23718 \mathrm{~N}$ & 4038 & 7023.2 & 22 Jun & 4134 & 7029 & $11 \mathrm{JuI} 56$ \\
\hline 76 & $23721 B$ & 4038 & 7023.2 & 22 Jun & 4138 & 7039 & 28 ปนา 56 \\
\hline 76 & $23722 \mathrm{~N}$ & 4038 & 7023.2 & 22 Jun & 4117.1 & 7014.7 & 4 JuI 56 \\
\hline 76 & $23723 B$ & 4038 & 7023.2 & 22 Jun & 4135 & 7049.5 & 22 JuI 56 \\
\hline 76 & $23724 \mathrm{~N}$ & 4038 & 7023.2 & 22 Jun & 4116.7 & 7013.8 & 2 Ju. 56 \\
\hline 77 & 237338 & 4005.5 & 7054 & 22 Jun & 3636 & 7550 & 200 ct 56 \\
\hline 78 & & 4006 & 7110 & 22 Jun & \multicolumn{3}{|c|}{ No returns } \\
\hline 79 & & 4016 & 7104 & 22 Jun & \multicolumn{3}{|c|}{ No returns } \\
\hline 80 & & 4025.8 & 7118.4 & 22 Jun & \multicolumn{3}{|c|}{ No $r$} \\
\hline 81 & & 3958.5 & 7144 & 22 Jun & \multicolumn{3}{|c|}{ No $n$} \\
\hline 82 & & $40 \quad 09.6$ & 7209.5 & 23 Jun & \multicolumn{3}{|c|}{ Nor } \\
\hline 83 & & 4000 & 7216.5 & 23 Jun & \multicolumn{3}{|c|}{ Ro $r$} \\
\hline 84 & & 3945.5 & 7232 & 23 Jun & \multicolumn{3}{|c|}{ No retums } \\
\hline 85 & & 3950.5 & 7247 & 23 Jun & \multicolumn{3}{|c|}{ No retums } \\
\hline 86 & & 4002 & 7303 & 23 Jun & \multicolumn{3}{|c|}{ No retums } \\
\hline 87 & $23846 \mathrm{~N}$ & 4018 & 7252.5 & $23 \mathrm{Jun}$ & 3506 & 7557 & 8 Nov 56 \\
\hline 87 & $23848 \mathrm{~N}$ & 4018 & 7252.5 & 23 Jun & 3625 & 7550 & 22 Oct 5 \\
\hline 87 & $23852 \mathrm{~N}$ & 4018 & 7252.5 & 23 Jun & 3954 & 7405 & 23 JuI 56 \\
\hline 87 & $23856 \mathrm{~N}$ & 4018 & 7252.5 & $23 \mathrm{Jun}$ & 3630 & 7551 & 18 oct 56 \\
\hline 88 & & 4034 & 7242 & $23 \mathrm{Jun}$ & \multicolumn{3}{|c|}{ No returns } \\
\hline 89 & & 4010 & 7232.5 & 23 Jun & \multicolumn{3}{|c|}{ No returns } \\
\hline 90 & $23888 \mathrm{~N}$ & 4017 & 7221 & 23 Jun & \multicolumn{3}{|r|}{21 oct 56} \\
\hline 90 & $23889 B$ & 4017 & 7221 & 23 Jun & 3601 & 7539 & 23 Oct 56 \\
\hline 91 & & 4039 & 7206.5 & 23 Jun & \multicolumn{3}{|c|}{ No returns } \\
\hline
\end{tabular}


Released

Station

92

93

94

94

94

94

95

95

95

95
Bottle

Lat

Iong Date

$4024.4 \quad 7148.8 \quad 23$ Jun

$4051.5 \quad 7128 \quad 23 \mathrm{Jun}$

$4100.5 \quad 71 \mathrm{IL}_{4} 23 \mathrm{Jun}$

$4100.57114 \quad 23$ Jun

$4100.57114 \quad 23$ Jun

$4100.571 I_{4} \quad 23 \mathrm{Jun}$

$417.5 \quad 7100$

4117.57100

$4117.5 \quad 7100$

417.57100
Recovered

Lat Long Date

No returns

$\begin{array}{lllll}41 & 21.8 & 71 & 37 & 2\end{array}$ Sep 56

$3909.5 \quad 744127$ Oct 56

$4136.2 \quad 7120 \quad 8$ Sep 56

$4119 \quad 7048.5 \quad 5$ Aug 56

$4739.5 \quad 6959.129$ Aug 56

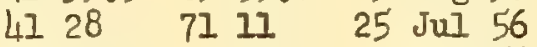

$\begin{array}{lllll}41 & 27.7 & 71 & 10.2 & 27 \\ 4 & \text { Jun } 56\end{array}$

$4127.7 \quad 71 \quad 10.229$ Jun 56

$4127.7 \quad 71 \quad 10.2 \quad 29$ Jun 56 
Appendix

Returns from 5 December 1956

to 21 October 1957

Released

Recovered

Station Dottle Iat Long Date Lat Long Date

ALEATROSS III Cruise 46

55

$12760 \mathrm{~N} \quad 4200$

6953

31 Iiar 53

4115.2

7010.2

6 iar 57

Cruise 48

31

$12927 \mathrm{~B}$

$\begin{array}{llllll}41 & 01.5 & 71 & 00.5 & 24 & \text { Apr } 53\end{array}$

$13271 B$

$42 \quad 14.5 \quad 70 \quad 34$

29 Apr 53

$4108 \quad 70 \quad 45$

25 Jun $57 \%$

$4007 \quad 7313 \quad 10 \mathrm{Jul} 57 \%$

\section{Cmise 50}

28

5

$16211 B$

$13927 \mathrm{~B}$

4146.5 60 i? 4024.5
30 liay 53 26 liay 53
Lí $40 \quad 0433$

$15 \operatorname{Jan} 57$ 40 l. $57254 \quad 21$ :ay $57 \%$

\section{Cruise 57}

18

27

$16314 \mathrm{~N}$ $16419 \mathrm{~B}$

4344 4245
$6900 \quad 24$ reb 55

$60125 \mathrm{E0b} 55$
5.) 45

.052

60,0

0122
$2 i+\operatorname{sar} 57$ 4 .ay 57

\section{Cruise 58}

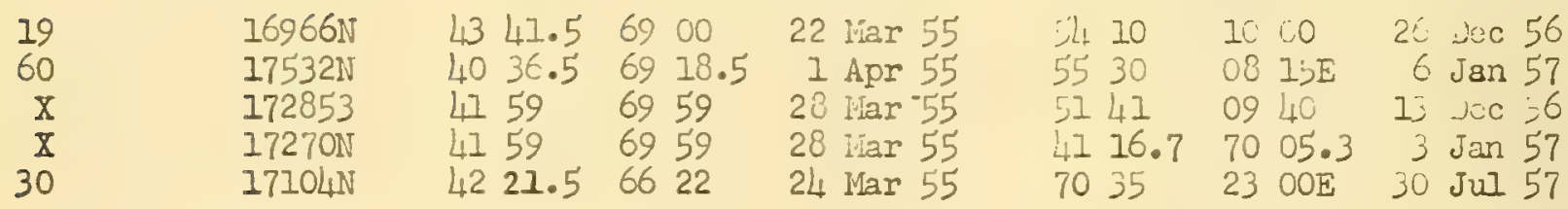

\section{Cruise 60}

\begin{tabular}{|c|c|c|c|c|c|c|c|}
\hline 9 & $17695 \mathrm{~B}$ & 4702.5 & 6729 & 20 Apr 55 & $53 \mathrm{I}_{4}$ & 0935 & 4 Feb 57 \\
\hline 64 & $28353 \mathrm{~B}$ & 4100 & 6849.3 & 29 Apr 55 & 6415 & $1015 \mathrm{E}$ & 5 Feb 57 \\
\hline & $18157 \mathrm{~B}$ & 4144 & 6920 & 27 Apr 55 & 4043.6 & 7253.1 & 14 JuI 55 \\
\hline 71 & $18435 \mathrm{~B}$ & 4101.5 & 6632 & 30 Apr 55 & 4748 & 0428 & 6 Feb 57 \\
\hline & $785 B$ & 4038.5 & 7007.7 & 3 Ifay 55 & 4120.7 & 7030 & 26 Mar 57 \\
\hline 8 & 17 & 4033 & 6741 & 20 Apr 55 & 6430 & $1125 E$ & 25 Apr 5 \\
\hline & 179118 & 4255.8 & 6933.8 & $22 \mathrm{Apr} 55^{\circ}$ & 4057 & 7022 & 20 Aug \\
\hline
\end{tabular}

* Bottle recovered from bottom 


Station Bottle Lat $\quad \underline{\text { Long }} \underset{\text { Cruise 61 }}{\underline{\text { Date }}}$ Iat $\quad$ Long Date

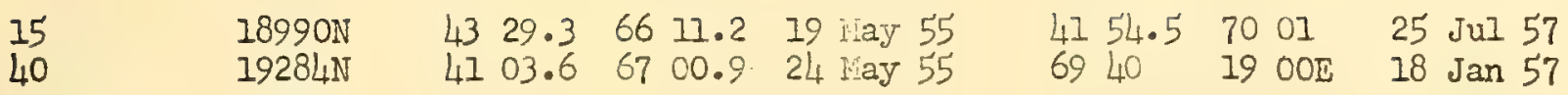

\section{Cruise 71}

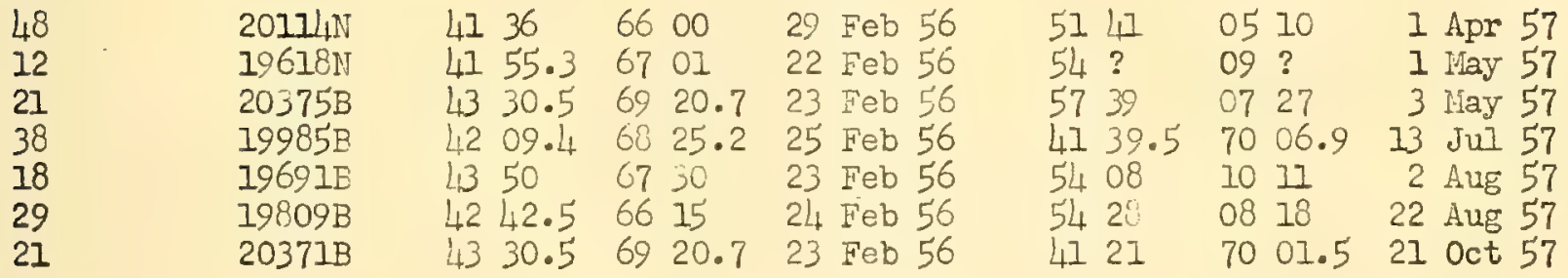

\section{Cruise 72}

12
21
31
17
30
9
15
22
22
37
42
22
2
15
29
33

31

17

30

22

37

42

22

2

15

29

33

$20458 \mathrm{~N}$

$20558 \mathrm{~N}$

$20691 \mathrm{~B}$

$20448 \mathrm{~N}$

$20687 \mathrm{~B}$

$20420 \mathrm{~N}$

20487B

$20569 \mathrm{~B}$

$20570 \mathrm{~N}$

$20770 \mathrm{~N}$

$20822 \mathrm{~N}$

$20576 \mathrm{~N}$

$20286 \mathrm{~N}$

$20490 \mathrm{~N}$

20671B

$20722 \mathrm{~N}$

\begin{abstract}
$4240 \quad 6622$
\end{abstract}
23 Nar 56 $4239.7 \quad 70 \quad 02$

24 Nar 56

$42 \quad 17.5 \quad 6602$

$28 \operatorname{Mar} 56$

4209

6654

$23 \operatorname{Mar} 56$

$\begin{array}{lllll}42 & 40.5 & 66 & 08.3 \quad 28 & \operatorname{Mar} 56\end{array}$

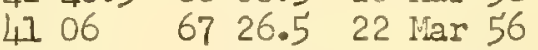

4259

4235

4235

6440

23 Mar 56

7023.526 Mar 56

4730

4106

4235

4038

4259

4246.5

4150.5
W $11 \quad 6610 \quad 24 \mathrm{Jan} 57$

$4148 \quad 6956 \quad 18$ Jan 57

$3220 \quad 6440.8 \quad 13$ Dec 56

$\begin{array}{lllll}5103 & 9 & 08 & 3 & \text { Apr } 57\end{array}$

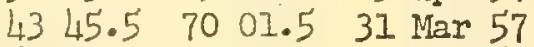

$\begin{array}{llll}6029 & 0138 \quad 1 \text { I liay } 57\end{array}$

$5630 \quad 0700 \quad 4$ May 57

$3754 \quad 7520 \quad 26$ May 57
7023.526 liar 56

6930

29 . Mar 56

6627

29 Mar 56

7023.5

26 I lar 56

7101

21 Mar 56

6440

24 Nar 56

5908

3745

5640

3620

3750

5348

$48 \quad 30$

4725

$0719 \quad 17 \mathrm{JuI} 57$

$2545 \quad 17$ Jun 57

$0630 \quad 5$ Jul 57

$7548.7 \quad 8$ Aug 57

$2530 \quad 16$ Aug 57

095824 Aug 57

$0450 \quad 15$ Sep 57

$\begin{array}{lll}67 & 02.528 & \text { Mar } 56\end{array}$
Cruise 73

14

17

32

55

65

15

45

56
$21105 \mathrm{~B}$

$2 \mathrm{I}_{4} 3 \mathrm{~B}$

$21320 \mathrm{~N}$

21583B

$21702 \mathrm{~N}$

$21716 \mathrm{~N}$

214573

$21590 \mathrm{~N}$
$4355.5 \quad 6651$

9 Apr 56 $\begin{array}{lllll}4329 & 7002.5 & 20 & \text { Apr } 56\end{array}$ $4233.47038 .822 \mathrm{Apr} 56$ $4218.6 \quad 6530.6 \quad 26$ Apr 56 $4048 \quad 7002 \quad 28$ Apr 56 $4350 \quad 6802 \quad 19$ Apr 56 $4034 \quad 6659 \quad 25$ Apr 56 $4146.3 \quad 6530.4 \quad 27$ Apr 56
$4512 \quad 6437 \quad 23$ Nov 56 $4448.8 \quad 6532.2 \quad 8 \quad J a n 57$

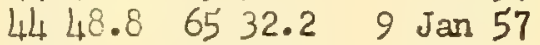

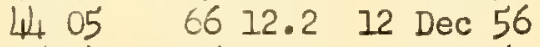

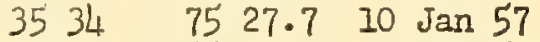
$4348 \quad 6609 \quad 22$ Feb 57 $5740 \quad 0620 \quad 13$ Feb 57 $\begin{array}{lllll}55 & 00.9 & 08 & 33.6 & 30 \\ \operatorname{Jan} & 57\end{array}$ 


\begin{tabular}{|c|c|c|c|c|c|c|c|}
\hline Station & Bottle & Lat & Long & Date & Lat & Long & Date \\
\hline & \multicolumn{7}{|c|}{ Cruise 73} \\
\hline $\begin{array}{l}57 \\
35 \\
31 \\
45 \\
62\end{array}$ & $\begin{array}{l}216041 \% \\
213501 \% \\
21310 \mathrm{~N} \\
21.50 \mathrm{~N} \\
21662 \mathrm{~N}\end{array}$ & $\begin{array}{ll}41 & 47.5 \\
41 & 34.5 \\
40 & 14 \\
40 & 34 \\
40 & 46\end{array}$ & $\begin{array}{ll}65 & 58 \\
69 & 04 \\
69 & 46.1 \\
66 & 59 \\
67 & 39\end{array}$ & $\begin{array}{l}27 \text { Apr } 56 \\
23 \text { Apr } 56 \\
22 \text { Apr } 56 \\
25 \text { Apr } 56 \\
27 \text { Apr } 56\end{array}$ & $\begin{array}{ll}39 & 23 \\
57 & 32 \\
41 & 55 \\
51 & 35 \\
39 & 50\end{array}$ & $\begin{array}{ll}31 & 18 \\
07 & 23 \\
66 & 50 \\
09 & 50 \\
31 & 10\end{array}$ & $\begin{array}{l}30 \text { Jan } 57 \\
19 \text { Arr } 57 \\
29 \text { Aub } 57 \\
24 \text { Ay } 57 \\
13 \text { iar } 57\end{array}$ \\
\hline
\end{tabular}

\section{Cruise 75}

8
10
39
45
52
27
47
65
20
61
43
5
144
39
75
62
74
85

20

22

25

26

28

45

93

1

36

45

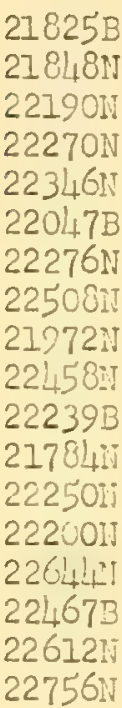

$4103 \quad 6717 \quad 17$ May 6

!1 $57 \quad 66 \quad 30 \quad 18$ May 56

$4242.46555 \quad 21$ Ilay 56

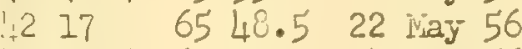

$\begin{array}{llllll}42 & 03.5 & 69 & 32.9 & 24\end{array}$ May 56

$\begin{array}{llll}14 & 51 & 6627 \quad 19 \text { lay } 56\end{array}$

$\begin{array}{lllll}42 & 11.5 & 65 & 02.7 \quad 23 & \text { May } 56\end{array}$

41 C. $5 \quad 6759.5 \quad 26$ May 56

4421

6721.8

19 l.ay 56

$\begin{array}{lllll}1.1 & 31.6 & 66 & 18.7 \quad 25 \text { liay } 56\end{array}$

$\begin{array}{llll}4 & 01.3 \quad 6657 \quad 22 \\ 40 & 0.5 & 68 & 56\end{array}$

$\begin{array}{llll}40 & 00.5 & 68 & 51\end{array} 17$ May 56

4220.9

6700

22 May 56

$121+2.4$

4018

6555

21 ilay 56

4118.8

6812

27 liay 56

4025.5

4003

$6728 \quad 27$ iay 56

$7200.3 \quad$ 2 i iay 56

Gruise 76

$23015 B \quad 4 C_{4}$ C2 E6 37.5 I4 Jun 56 $23035 B$ i山 $24 \quad 6642.5$ 14 Jun 56 $23043 B \quad 44446600 \quad 14$ Jun 56 $23076 \mathrm{~N} \quad 4500.86607 \quad I_{4}$ Jun 56 $23104 N \quad 4413.5 \quad 6658 \quad 15$ Jun 56 $231798 \quad 4358 \quad 6734 \quad 15$ Jun 56 $23 \nu_{11 B} \quad 4346.5 \quad 6840 \quad 15$ Jun 56 $23349 B \quad 4128 \quad 6726 \quad 18$ Jun 56 $23924 \mathrm{~N} \quad 4051.5 \quad 7128 \quad 23$ Jun 56 $22816 \mathrm{~N} \quad 4041 \quad 7101 \quad 11$ Jun 56 23001B 4j35 $6703.2 \quad 13$ Jun 56 $23243 B \quad 4245 \quad 6619 \quad 16$ Jun 56 $23321 B \quad 4126.46601 \quad 17$ Jun 56

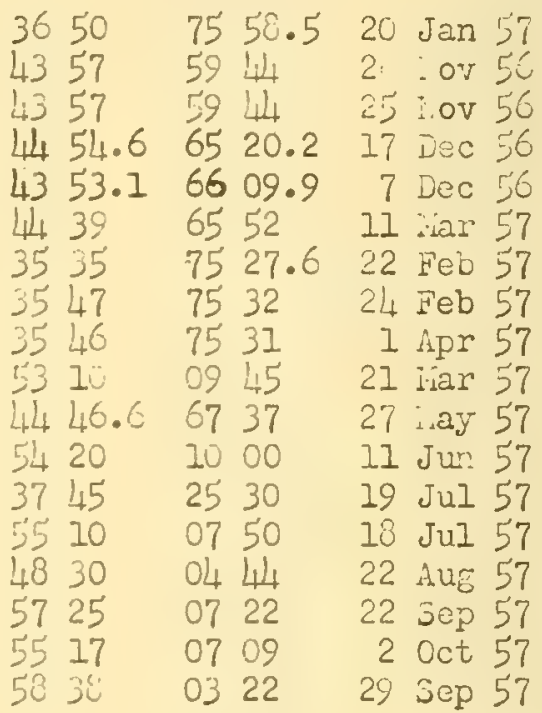

$4144 \quad 7038 \quad 8$ Dec 56 $1417.5 \quad 6608 \quad 17$ Feb 57

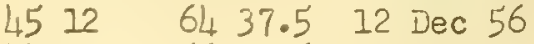
$44 \quad 17.2 \quad 6620.6 \quad 22$ Dec 56 $\begin{array}{lllll}45 & 00.5 & 65 & 09.3 \quad 8 & \text { Dec } 56\end{array}$ 44 $05 \quad 6612.8 \quad 21 \mathrm{Jan} 57$ $\begin{array}{lllll}4 & 23.4 & 66 & 03.8 & 16 \\ \text { Jec } & 56\end{array}$ $4454.5 \quad 6521.4 \quad 2 \operatorname{Jan} 57$ $3622.6 \quad 75 \quad 49.4 \quad 15$ Dec 56 Virginia Coast 9 Mar 57 $4347.7 \quad 6620 \quad 25 \mathrm{Feb} 57$

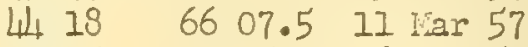
39253115 6 Lar 57 
Released

\begin{tabular}{|c|c|c|c|c|c|c|c|}
\hline tation & Bottle & Lat & Iong & Date & Iat & Long & Date \\
\hline $\begin{array}{l}29 \\
41 \\
74 \\
77 \\
22 \\
1 \\
61\end{array}$ & $\begin{array}{l}23158 \mathrm{NI} \\
23304 \mathrm{NI} \\
23692 \mathrm{~N} \\
23731 \mathrm{D} \\
23066 \mathrm{~N} \\
22824 \mathrm{~N} \\
23536 \mathrm{~N} \\
23890 \mathrm{~N}\end{array}$ & $\begin{array}{ll}43 & 39 \\
42 & 04.8 \\
39 & 53 \\
40 & 00.5 \\
45 & 00.8 \\
140 & 41 \\
41 & 05 \\
40 & 17\end{array}$ & $\begin{array}{ll}69 & 30.2 \\
65 & 34.1 \\
69 & 42.6 \\
70 & 54 \\
66 & 07 \\
71 & 01 \\
67 & 22 \\
72 & 21\end{array}$ & 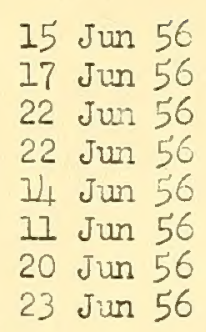 & $\begin{array}{ll}43 & 51 \\
55 & 10 \\
35 & 33 \cdot 9 \\
36 & 22.5 \\
45 & 17 \cdot 5 \\
34 & 55 \\
57 & 07 \\
35 & 22\end{array}$ & $\begin{array}{ll}69 & 59 \\
06 & 47 \\
75 & 27.7 \\
75 & 49.4 \\
6 ! & 46.5 \\
76 & 11 \\
69 & 45 \\
75 & 29.6\end{array}$ & $\begin{array}{rrr}27 & \text { lay } & 57 \\
8 & \text { Jun } & 57 \\
11 & \text { Jun } & 57 \\
25 & \text { Jun } & 57 \\
15 & \text { Jiay } & 57 \\
11 & \text { Oct } & 57 \\
25 & \text { Sep } & 57 \\
9 & \text { Oct } & 57\end{array}$ \\
\hline
\end{tabular}



